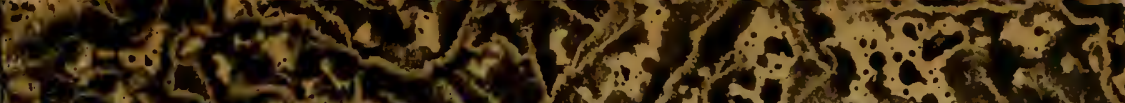

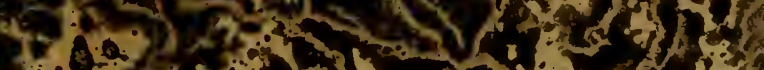

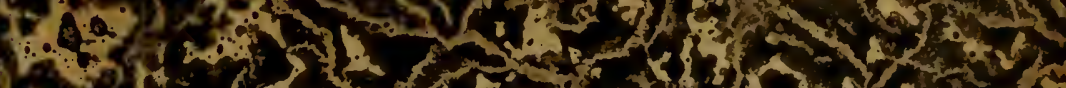
(1)

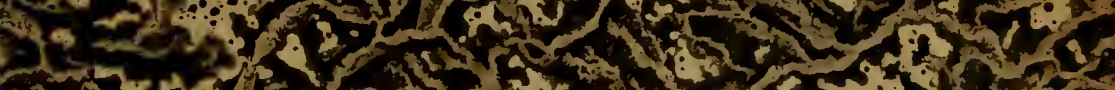

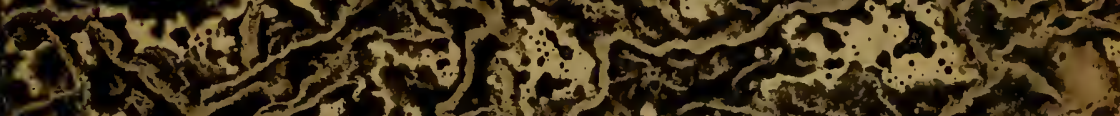

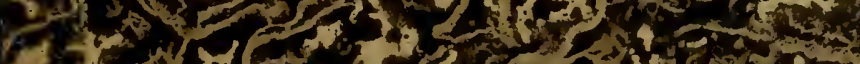

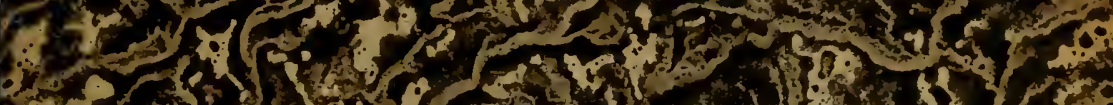

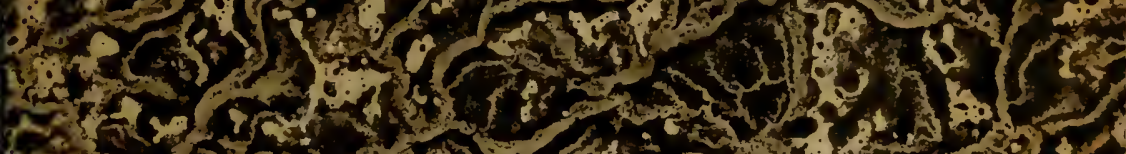

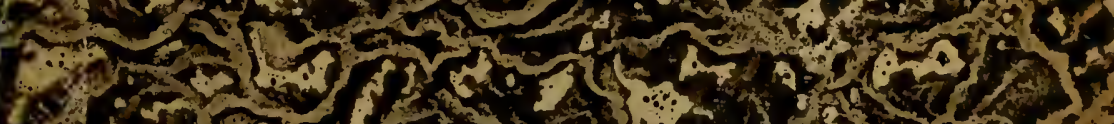

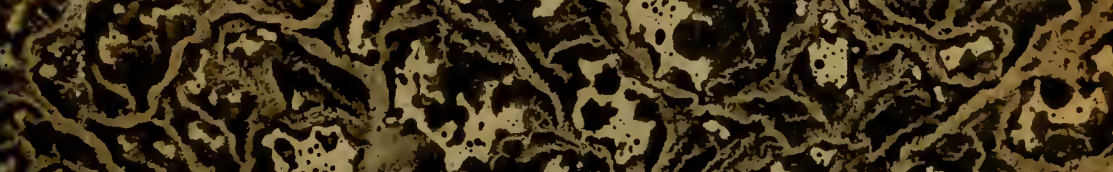

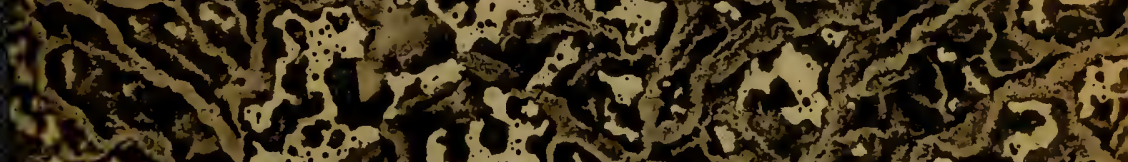

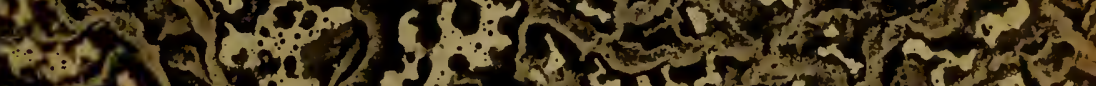

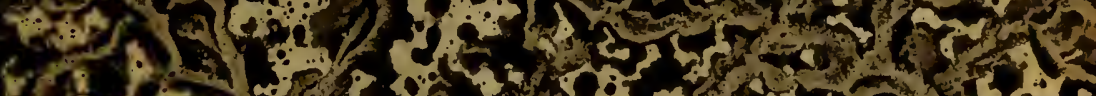

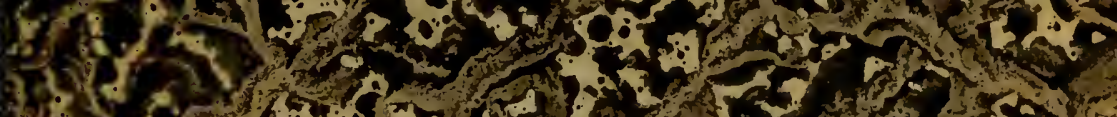

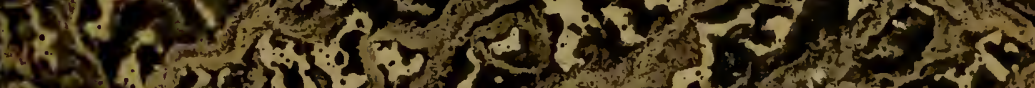

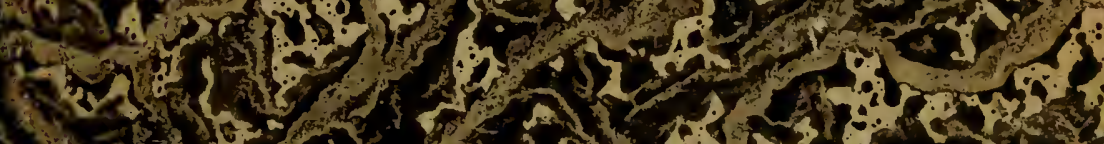

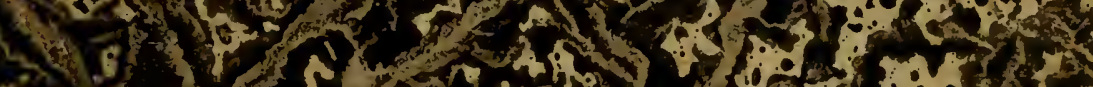

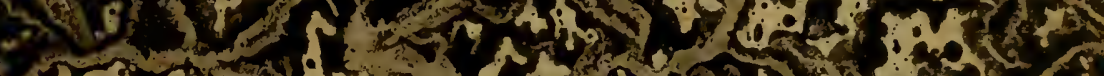

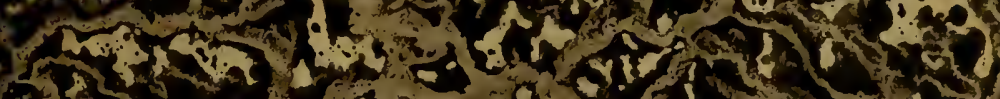

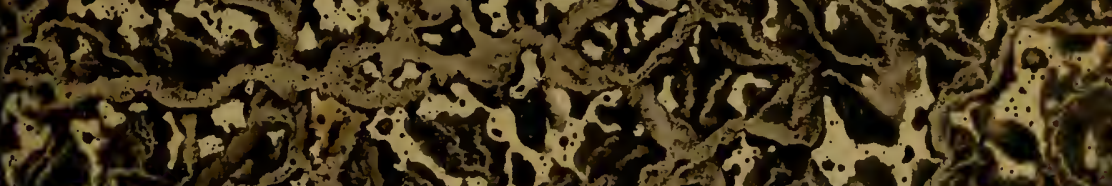

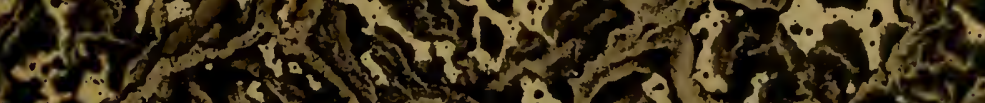

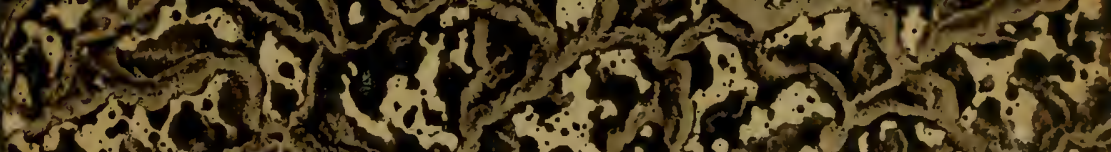

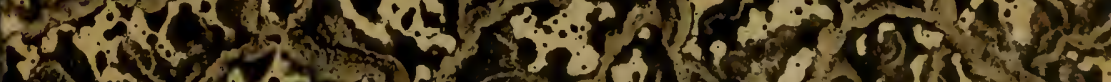

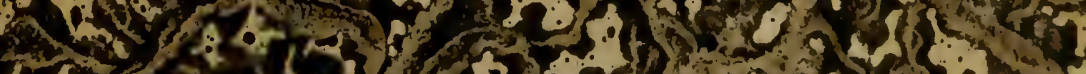

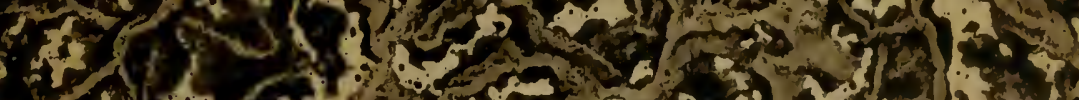
(5) tw 


\section{Pifibrary of the Gefluscum}

\section{OF \\ COMPARATIVE ZOÖLOGY,}

AT HARTIRD COLLEE, CIIIBRIDGE, JIASS.

Jounded ty pribate subscription, în 1861.

DR. L. DE KONINCK'S LIBRARY. No. $.55 \%$ 



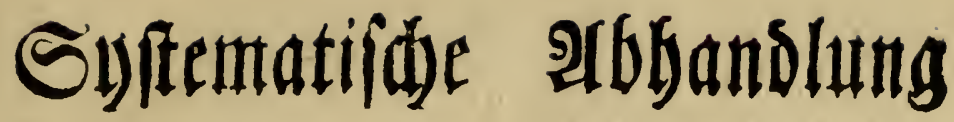

น⿰ $\mathfrak{b}$ e

\section{Dic (Et) : und FIußcondifilien}

welde

um Auggburg und Der umllegenden Esegend gefunden werden.

\section{צlB ein $\mathfrak{B}$ entrag}

zur vaterlándifaen Naturgefdidate

$\mathfrak{v} 0 \mathfrak{n}$

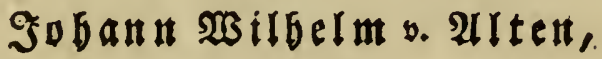

Doftor ber Philopopbie, Mag. Der fr. Surnfte, 2ppotheler zunt golbenen Engel in 2 lugaburg, aubivártigem vortragendem Mit: gliede ber Şallif bem Mitgliebe ber 2 Betterauifden Befellfdaft für bie gefammte

शtaturfunte und EGren = Mitgliebe Der botanifđen

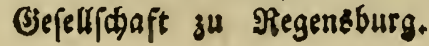

Mit 14 Nupfertafelti.

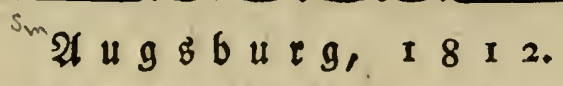

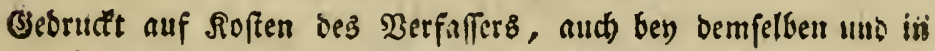

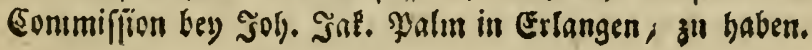





\section{Deit}

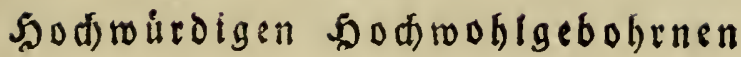

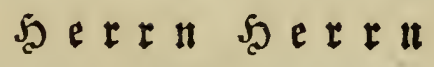

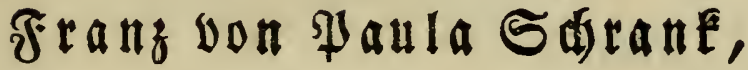

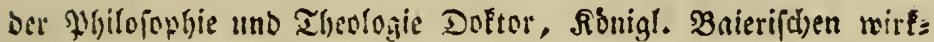
líd)en gefleimen geiftlichen Stathe, nf́tiven Mitgliebe ber Rónigls

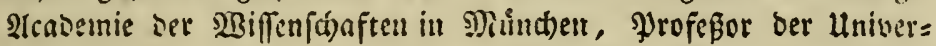
fitit, Der Defonomie uno ofonomifiten Botanif zu Sngolftast,

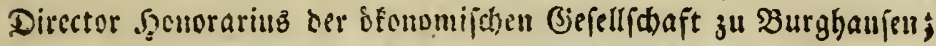

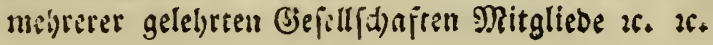

\section{Dem wohlgebobrnen

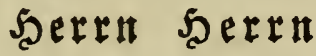 Dr. Sottfried SSätner,}

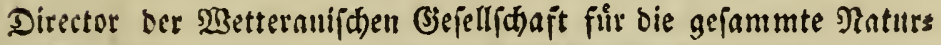

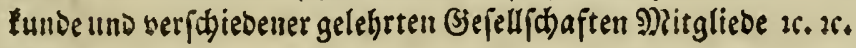

\section{Den wohlgebohenen

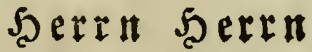 $\mathfrak{A} \mathfrak{H} \mathfrak{g} \mathfrak{H} \mathfrak{t} \mathfrak{A} \mathfrak{L} \mathfrak{e} \mathfrak{H} \mathfrak{B}$,}

pribatifirenten 9haturforider uno sortragenden Mitgliese ber

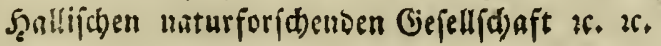



In minimis maxima Sapientia.

Geoffroy.

\section{Einleitende Bortede.}

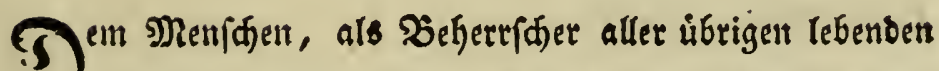

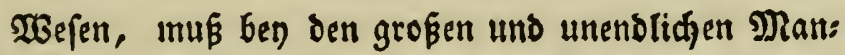
nigfaltigleiten, weldhe ifm bie unerfajpflidje Natur in ifren 3 Reiden barbiethet, nidhts angenehmers fenn, als auf ber einen Seite bie berrlidgen Begenftånde, weldje fin ifm barbiethen, bis auf bas leinfte unb unbedeu: tend fheinenbe entwidelt unb gleidfam vor fid) ausgebrei: tet zu fehen; auf ber anbern Seite aber, biejes fid noth: 


\section{$=\mathrm{vi}_{\mathrm{I}}=$}

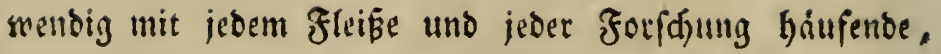
mógltáfí anteinanber gereiht uno in eine leidft úberfegbare Dromung bargeftellt fu fehen.

Naturfimbe wito, fo lange Menfacen auf biefer Erbe

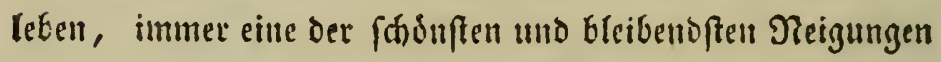

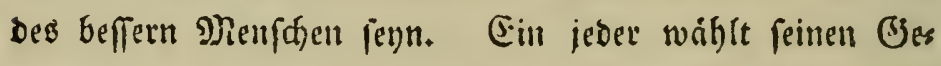
genftand: id menne eigenttiá) den Naturforider, une nidgts ift ifgm zh Elcin,

Der Naturforfater von Yrofoffĩon, swemu id midh fo alsorúcfen barf, fammelt nicht allein, er eronet aud,

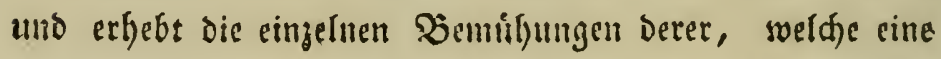
genvife गieigung nuf biefes ober jenes leitcte, fu eimem

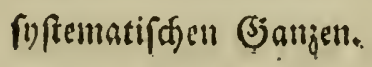

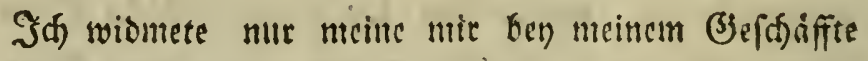
siberbleibenden fparfamen Ticbonfuntben, einem Sleeile ber Daturfunbe, ber fifon vicle vor unir befordfftigte, wie

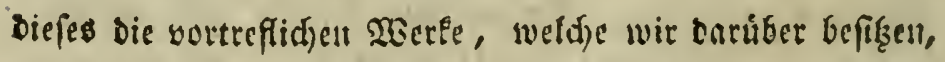
zeigen; id war bingejegen an bie rubigen, unbenerlten Berwobuer unfezcr Erte, Dereat grópter Fgeil von ben 
mefreften, felbft gebilbeten Menfdjen uidst beadjtet, obet robl gat veradtet wirb. Die Eondilien.

Die Begend um Hugsburg ungefäbr 4 bis 6 Stun: in Umfang ift jiemlid, reich an jenen intereffanten $\mathfrak{B}_{e}$ soofuern ber Eroe uno ber Waifer.

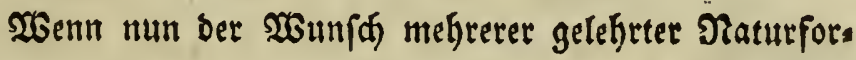
fajer, befonders berjenigen, weld)e fid um bie Condillo: logie bleibende Bersienfte erworben, einmal eine volls ftandige beutjaje Condiliologie z̆l begrunden - realifirt werben foll, fo ift es nothwendig, dafi jeber, den biefe Sadje intereffirt, feit Sdärflein bazu beytrage und nad) Siråten feine Begend gleid) (aim ausfubje.

Der großße Naturforfaer, Şerr Yaftor Sdáffer, äu: Berte fin über Diefen - Segenftanto in. feinem "Berfude mit Sanecfen 2te Auflage" pag. 6I, Gey feiner 5tan Sdlukammerkung folgend:

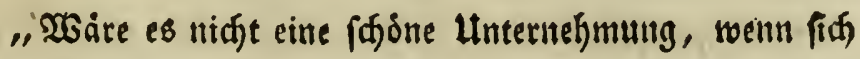

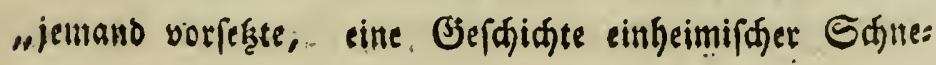


"tfell, fowohl was ihre Şälfer tund Sdjalen, als was "ifre thicrifd)e Beftalt betrifft, in ausgewåh)ten Siupfers "ftichell zu liefern? SBarum bleibet man bieffalls nur "bev) ausländifayen Sdjnecten uno Sdyalen fethen, uno "leget fie in Die Draturaliencabineter? Die einbeimifdjen "find biefer Elgre gewißß nid)t unwuirdiger."

Shne anmaffend ober ftolg zu fenn, glaube ids mit

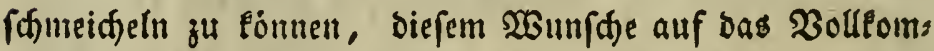
menfte entfprodjen zu baben, id bente ber Dpfer unto Sdjwierigéeiten nid)t, welde bey diefem Biegenftande ber

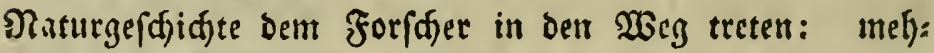
rete muibevolle Tage bringen oft nidt ben geringlten Funt, uno biefes mag aud wohi bie Urfadpe fenn, warun biefer nur in feinem längen 3 erfolg intereffant werbente Begenftand fo wenig 20refiuer hat, wb uns gleid) bie bunten Sthalengebåupes Eammlung ber Sublänber tiber:

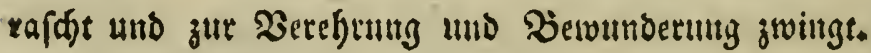

Dren Jalke lang babe id, fo viel mil meine Mule geftattete, Diefen (3)genftand verfolgt mo dic angegebene

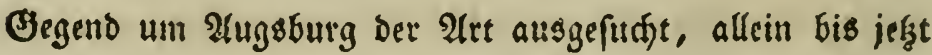

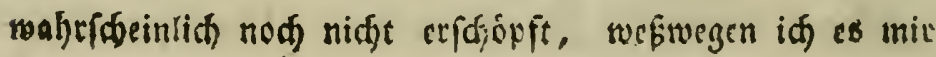




\section{$=\mathrm{Ix}=$}

vorbehalte, Diejenigen Epecies, welde in ber Folge nod aufgefunden werben follten, in irgent ciner 3ritfdrift als Nadftrag zu liefern.

ЭФ) bejarieb biet 58 Species und liés 26 berferfen abbilben; Der Simfter uno Naturalienmahlex, Şerr Jo: feph Jgnaz Şdrmann, welher mir bie Zeidnumgen lie; ferte, ift feloft ein febre aufmerfiamer, aufpemdjlofer $\mathfrak{T a}$ : turforfder, dem id) biefes (jefdaffit vollfommen anver: trauen lonnte, uno weld)er nidft blok medjanifa fondern wiffenfdhaftlid ju wacte gebt.

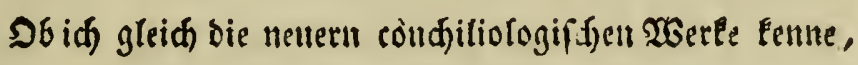

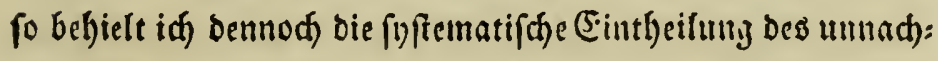
afmlid)en, unfterblid)en Linné ben, weil fie mir bie lcidtefte

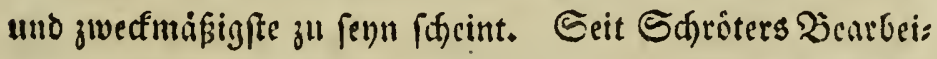

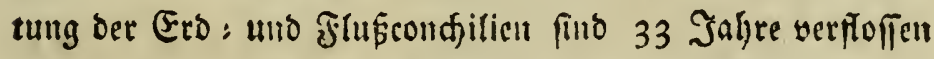

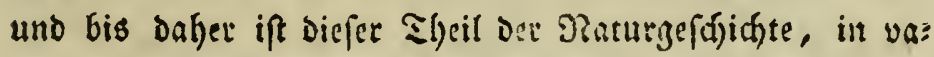

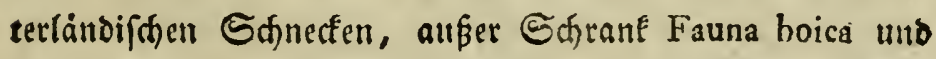
Sturm Deutfhlands Fauna fapt ganz vertachlápiget wor: Den, baher befuits)te idh nid)t, Daßs meine 2 (tbeit firr un: nifg, ober gat fir úberflipigig gebniten werdun follte. 


\section{$=x^{\prime \prime}=$}

Shan bat fid viefurefir lieber mit ben auslanbifden Sdjitecten befdáfftiget uno wabrfdeinlid aud befwegen, sweil fie urber in bie $\mathfrak{A}$ ugen fallet, und ifge Struftur fo:

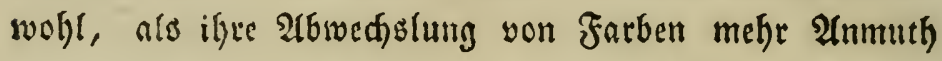
gewabren, was freylid) bey ben inläbifden nur felten ber Fall ift, bief mód)te aud Urfadje (ên, warum man fid bisher fo wenig ghüge gegeben hat, eigene Sammlungen von unfern cintheimijagen anjulegen.

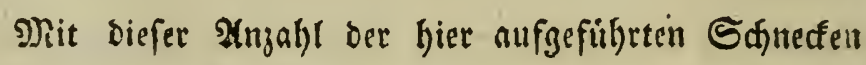
mo sjunfádeln, halte id nitht bafür bie fämmtlid)en in biefiger Girguro wohnenden alle bereits gefunden zu haben, es fitto mit noá) eitrige befannt, Liber beren ßeftimmung id) aber bis jefst nod) zweifclfaft bitr. Bề ber Beftim: mung bin id) aufs forgfältigfte zu Werke gegangen uno babe da teire neue Nafmen gegeben, wo fdou alte vor: hanben waren, aller bey gall neuen $\mathfrak{A}$ rten, wo Peime Defareibung vollemmen zutraf, beswegen babe idf ges flieffentlich vermicben, folde zll wäblen, welche in Ein: meifden Soften vorfommen, bie ueuen 2 rten aber in bie Abthcillutg gebrad)t, wohin fie nad) ibrer Etruftur ge: foren. 


\section{$=x \mathbf{x}=$}

Şwar haben cinige Sdriftftelfer bie Shale mit bem

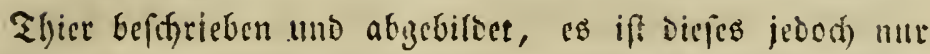

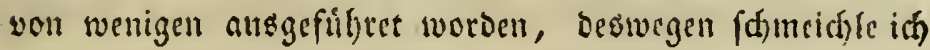

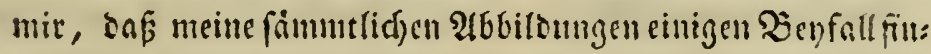
sen werden: um fo uchr, ba fie mit Der meglidffen Zartheit

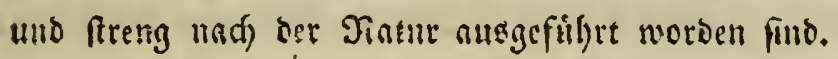

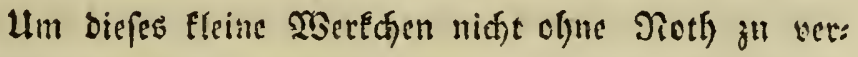
grókern unb ju vertbeuren, habe -id) nid)t fímmtliche Ero : und Flupfdyucten in Supfer frechen laffen, fontorn nur biejenigen, weldhe zwar befannt, aber in leinem PBerte in Supfer erfdienen futb, oder dic sabbildung jum Sheil uneenutlia uno ofme sas ?hict yorbanton ift; Dann abar

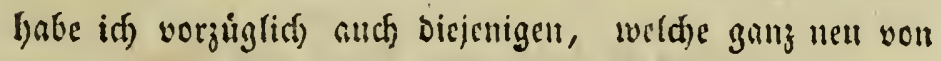
mir entocft worben fino, abbirben loffur. Ben anderen, nie j. $\mathfrak{B}$. Helix obscura mo. Helix hispida hatte if bie

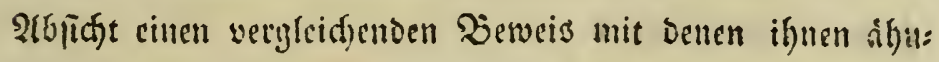
lidjen Helix buccinata uno Helix pilosa barzufteffen, bie Helix fascicularis aber ließ̧ ing nidyt wegen ber Sajale,

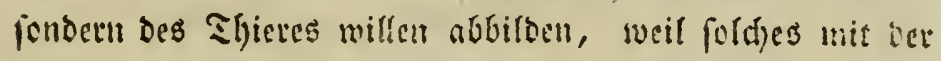

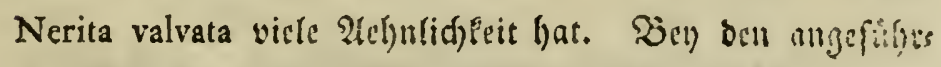

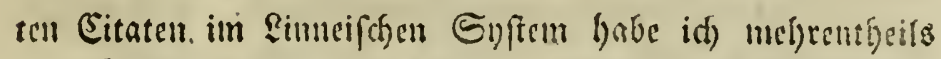

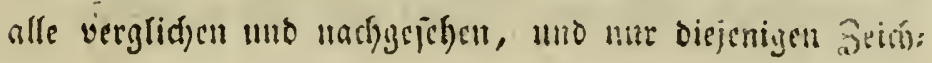




\section{$={ }^{x 11}=$}

numgen angegeben, welde am Penntlidfitent waren, Gey cinigen, wo biefes nidgt ber Fall ift, habe id bem Eitate Bemerenungen bengefügt. $\mathcal{D a}$ Múllers Historia

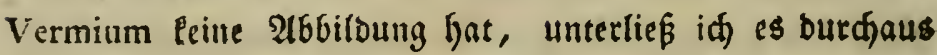
Sitate jul maden, war aber bernod genothiget biejenigen Sdunefiell, weldhe von Miller entbecft und befdrieben Finto, bier anzuführen. Die Terminologie fủr bie Sagals wuitmer wurde aus mehreren Sdyriften entlehnt, ba id

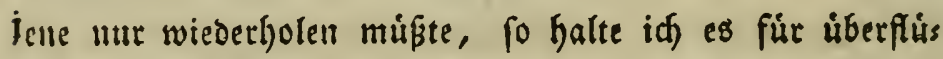
fig lie biefer $\mathfrak{A}$ b乌andlung angufügen, bem es aber baran

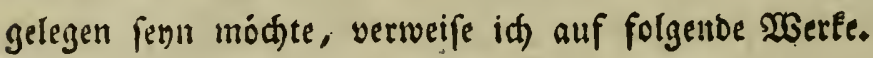

Linné Termini conchiliologici, von Johann Samuel Edjriter.

Berlinifhes Magazin 2ter Band $4^{\text {tes Stúc. }}$

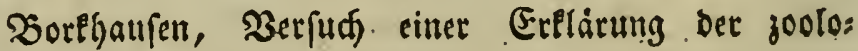
gifden Termintologie.

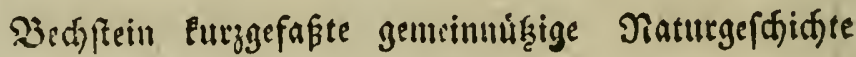
Iffet $\mathfrak{B}$ andes ate $\mathfrak{A}$ btheilung.

Glliger's Zerfud) einer fnftematifden, vollfänbigen

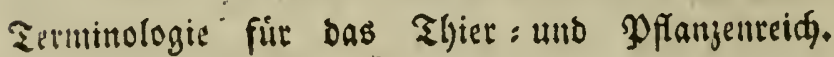




\section{$=\mathrm{x}_{111}=$}

als meine erfte $\mathfrak{A}$ rbeit, werben billige Sititifer nad): fidtig fenn, um fo mehr, oa meine befajränte Seit แmo viele unvorauggefehene Sdrwierigkeiten mir in Den $\mathfrak{W}_{\mathbf{S}}$ traten, 10, baß̧ idf bey vieleu 2 (ufopferungen unb $\mathfrak{A} 11=$ ftrengungen 3 Jahre Damit zubradgte und meine 26 fidst biebey nidfts weniger als Eigennus ift. गlod glaube ids

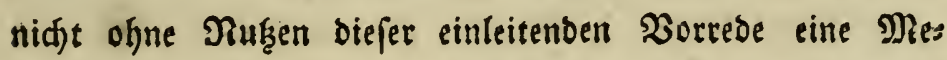
thobe benzufigen, wie bie Condilien am beften gu reinis gen finb, um fie lange fajön und gut mit $\mathfrak{B} e$ bbebaltung ihrer Farben-und ibres Blanges in Sammlungen aufzus bewabren. Die Erofdnecfen namlid werben in Waffer gelegt uno fo lange barinnen gelaffen, bis ber ßewobuer ganj heraus unb aufgefdrwollen ift, alsbann nimmt man bie Sdnecfe und wirft fie fonell in fiebendes waffer, weil bas Fhier báburd plokglid ftirbt unb fich nidjt wie: Der zurúcfiegen tann, fo roirb es leidgt zu faffen uno langfam, Damit es nid)t abreift, alts ben $\mathfrak{X}_{\text {Sintoungert }}$ berauszuziehen fenn. $\mathfrak{b}$ en ben 2 afferfanecfell Eann bieje

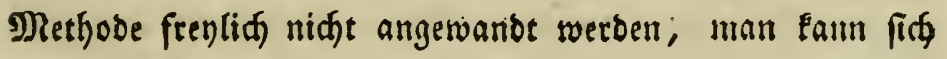
aber ebenfalls bes fiebenden waffers bebienen unb bey gar fu leidgt zerbrectlichen, wie Bulla fontinalis und einigent anberen bie 3eit wåklen, wenn fie iffe Jungen zur welt bringen, weil fie ba ifre CSebăupe verlaffell, ober wo biefes nidjt der Fall ift, muß man ben Sewolynet 


\section{$=x \mathrm{xIV}=$}

ait einent tempetirten Drte vertucfuen laffent. - Die Edale gewimit bnourd, wem bas FGier rein hernuts genommen worben, Hicht mur allein ein beffers Ifnjefyen, fonbern ift aug Dem Betderben ntat unterworfen. Die Sqale aber yon Sdumus uno Unreinigeeiten zul be, frenen, bientet eine Suirfte, beffer aber Sdadjtelhaln (Equifetum hiemale) weldem auf einen Sdleifirein die Dianbigkeit abgerieben ift, mit biefen wird alsoann wie

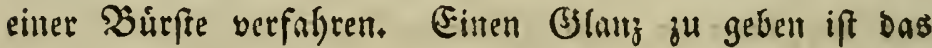
befte sittel, fie mit einem feinen Tud) oder Eeder abzus reiben uno madjoem aud inrwendig bie swindungen gans ausgetrocfinet fint, fie in Pleite Siapfelt vou trocfentut

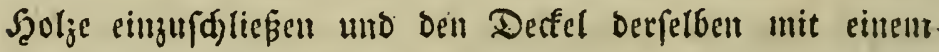
uhrglafe zu verfeben, basurdh fito fie gegen bie Einwil: fung aller etiva idadoliden Einfliffe, wié Staub, \&uft

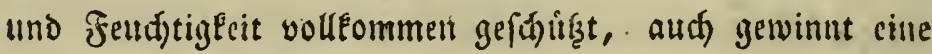

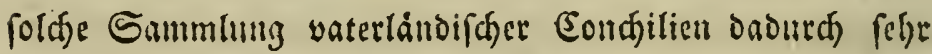
viel, fo, Daj fie mir, neben-Dem bunteften Cabinet memer erotifanen Contbilien wahres ßergnugen gewáftet.;

3wat find bie fier zแ Eearbeitenden Daturgegenftånde

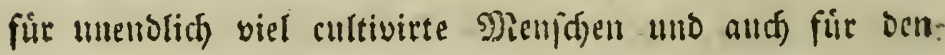

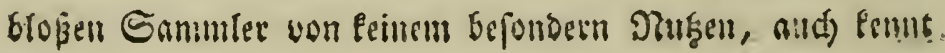


Der Naturforfder nid)t einmal iffen Zెweff, wartim. fite

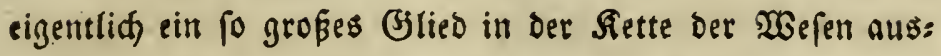
maden, fie geldoren aber bennod) ju ben berwubernngs: whroigen Biefaopfen unfer Erde, und eben-biefes ift ia

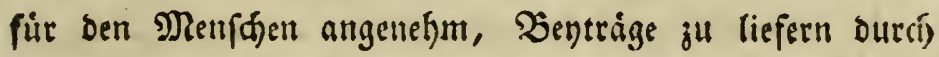

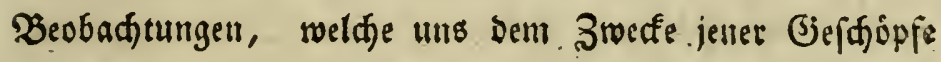
nåber Gringen.

Dáp bie Sdunecten feleft fowoh! als beren Shaten in 2(bfid)t ifher Farben von ein und derfelben Species fajon in einer Begent yon $2-3$ Stunden im $\mathfrak{L}$ mange merelid abweidjert, hat faon mander Condiltologe vor mir bes merlt, wie viel weniger ift fid zll wutudern, soenn foldse in groferer Entfernung nod weit mebr von cinander ab: reichen, weil bas Clima allerdings bierauf Einfluz bat; fo roie 3. $\mathfrak{b}$. bie italienifáfe Helix pomatia von unferen

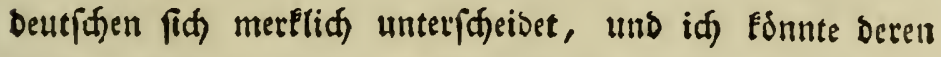
nod) melyrere anführen, allein id, bin vibergengt bns bies Pez ein jeder Naturforfajer, weld)er fí mit biefen (j): genftänden befdáfftiget zur Benủge bemerlt நat, unb ju jebergeit beunerlen roiro. 


\section{$=x \mathrm{VI}=$}

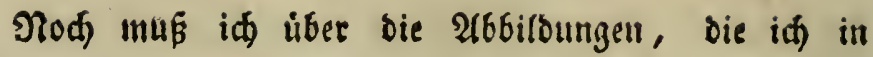

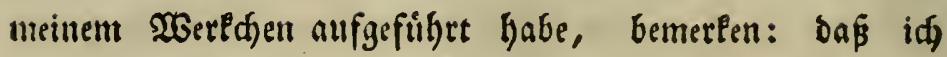
mefrere Sdinecfen gerabe auf Dem Biggenfiano habe abbils ben laffen, auf weldem fie gefuttben werben, wie f. $\mathfrak{B}$. Helix lenticularis auf Lemna trisulca L. Helix pilosa auf einem 20 eiderbuifaggen (Salix molissima) Helix thymorum auf Thymus ferpillum. Diefe $\mathfrak{A}$ noronung ift bem $\mathfrak{A}$ uge felge gefällig uno giebt ber ganzen Sadje mehr Eeben. 


\section{Mya pictorum. Linn.}

\section{Die spofletmufdel.}

M testa ovata, cardinis dente primario crenulata: laterali longitudinali alterius duplicato.

Linn. Syst, Nat. pag. 3218. Sp. 3.

Gualtieri Index Test. Conch. Tab. 7. Fig. E.

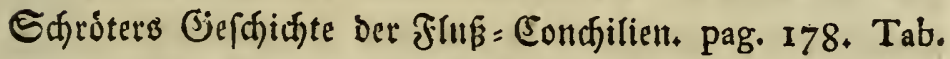
2. Fig. 2. 3, und Tab. 4. Fig. 6 . Chèmuiz Condjitien: Cabinet 6. Band. Tab. I, Fig. 6, Sturm Dentfoflanos Fauna 6. Aftheilung. Die Woirmet. 2. Şeft bas 13. 14. ulld 15 , Silufer.

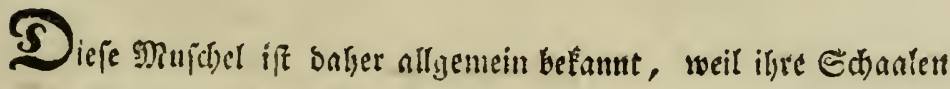

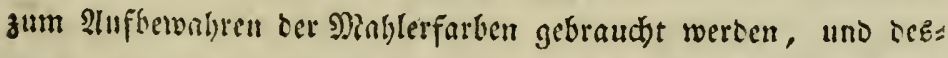

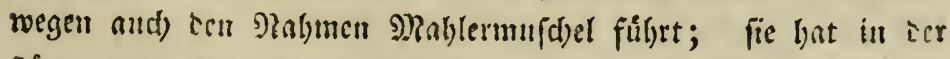

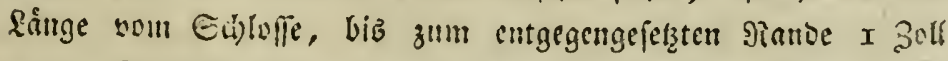
und 8 finien, uno in ber Sircite $3 \frac{3}{4}$ 3oll \$arifer Mañ. Eis

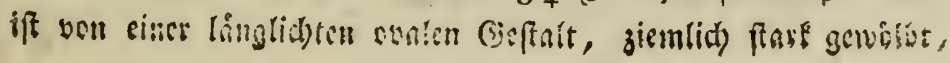




\section{$=2=$}

unten am Ranbe wenig eingebogen, biffid)aalig uno fanser, glimzeno, faft glatt, unturd)fid)tig, nut ben Edumibeln aber ourd)f(t)einend, uns mit bogeuformigen Duerfurchen und Etrcifen

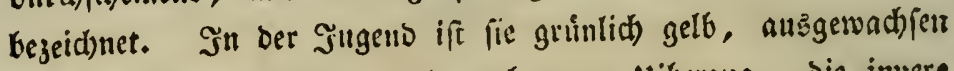

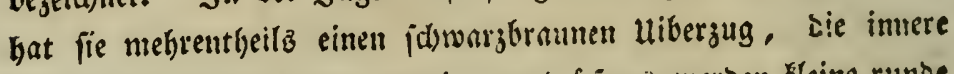
Seite ift weiß perfenmutterartig, uno offers weroen Fleine runde Perlen in ifr gefunoen. Die Sd)nabel fino wenig herborragento etwab getrogen, und allemahl ift iffe Epidermis abgerietert. Das Edbló liegt auper bem Mittelpunft "nă) Der obern breiten

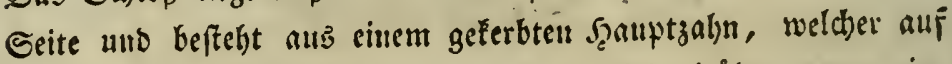
ber anbern linfen Sajaale in 2 geferbte Mitteljåline genau ein= fidlię̧t. Unter bem mujculófen Bande, wield)eb cine rotbbraune Garbe hat, hefindet fid noch an ber redhten Ed)aale ein feljr werlångerter fharfer Seitenzabn, weld)er in bie långlid)e Ber= tiefung ber entgegengejetzten Sdaale genau eingreift. Die Ceiten firto felfr ungleid), bic sordere hieson verlängert fid) in eine ftumpfe Ecee, Die bintere ift fehr furz uno abgerumbet.

Der Bewohnet hat eine grauliche Farbe, uno lommt mit bem Mytillus Anatinus in $23 e$ enttlidjen riberein.

In unjer Biegetto finoet fie fid) in ber Edjmutter uno in

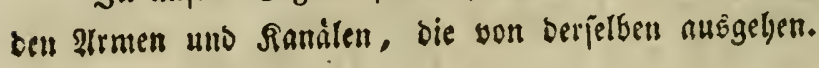




\section{$=4=$}

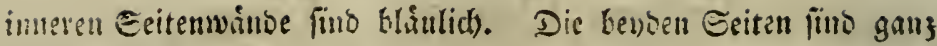

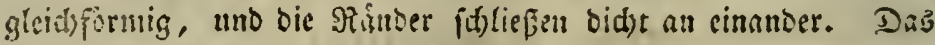
巨d)!of lisgt gerabe im mittelpunft, uno bat vorne uno bius

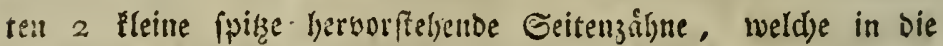

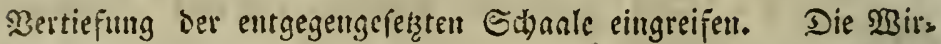

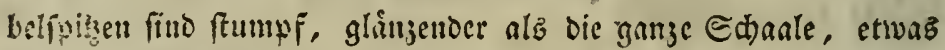
abgerieben uno geflefft, fie erfdyeinen bey manden subivisuen gleidy) faun wie runbe 2 tugen.

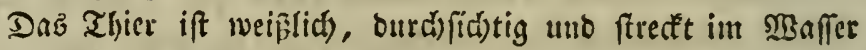

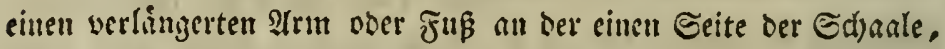
uno in ber entgegengefegrten 2 wentig verlängerte Sautgriffel heraub, bie fich imbenbig vereinigen, uno wowon ber eine etwas z":gefpitzt, ber anbre aber: eine runbe ftumpf s lappigte Minoung

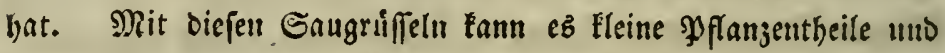
andere (jegenftánbe an fich) zieflen, bie ilgm zn feiner Nahrung bieneit.

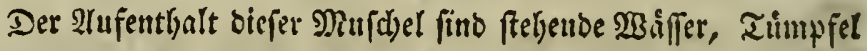

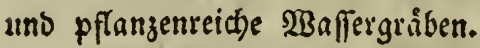

\section{Tellina tuberculata. Mihi. Tab. r. Fig. I. Die Şơder Telline.}

\section{8}

S.) habe biefe Mufthel mit anberen Tellinen auf bas Sorgfill, tigfte verglident unb mid tibergengt, oaß fie fowogl sutrd bie wejentlich)iten Relunzeid)an, als: Sanart, Garbe uno grópere

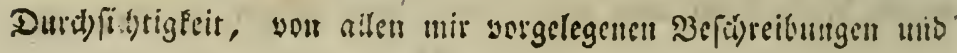




\section{$=5=$}

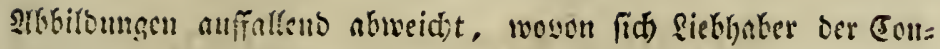

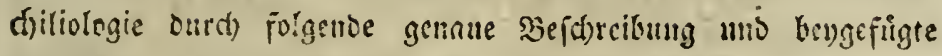

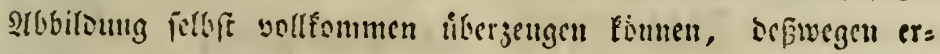
finme id) fie fuir cine nime Epecits mo gab ige wegen ben an

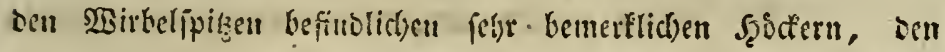

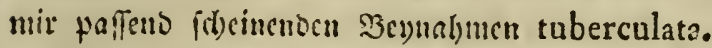

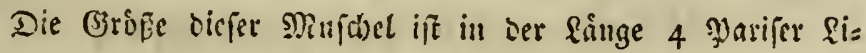
nicn, in sci Breite volttommen 5 finten uno in ber Şjhe 3

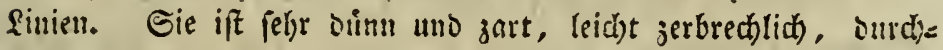
firi)tig, glinzent, fcin geftreift, sidffưrbig ins gelbliche fpie= lento, etwas flad) und an bem Sinnoe wo fidi) bie ecthalen

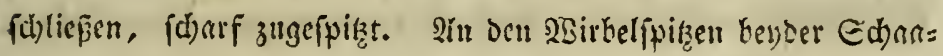

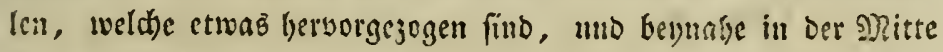

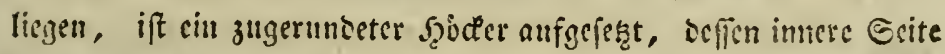
bohl ift. Die. Eciten fino etmas ungleid), bic ciare bitoct num

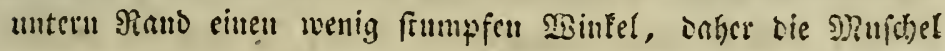

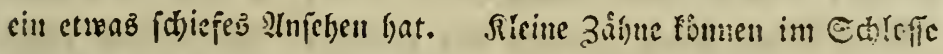

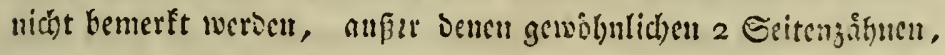
weld)e aher etwas flcinet fino als bey ber Tellina Cornea.

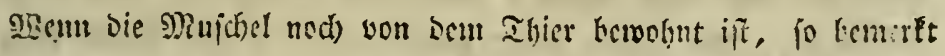

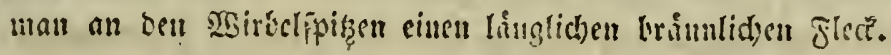

Das Thier ift woiplidn, surdfichtig, uno foum mit ier Tellina Cornea jo jientid ibersin.

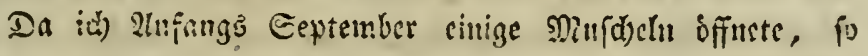

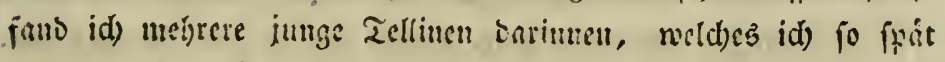
nie vermuthet batte; - fie get)ort Demnach) wie bie Tellina Cornea unter bie febentiggetsalgrctben.

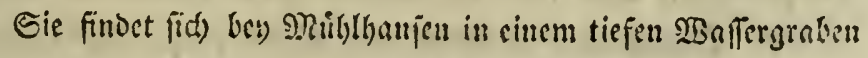

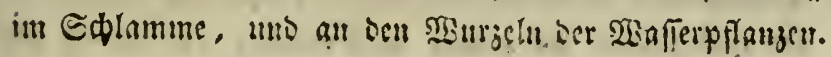




\section{$=6=$}

\section{Mytillus Cygneus. Linn.}

\section{Die großpe Ieidmuldel.}

M. testa ovata anterius compressuiscula fragilissima, cardine laterali.

Linn. Syst. Nat. pag. 3355. Sp. I5.

Gualtieri Index Test. Conch. Tab. 7. Fig. F.

Elyenniz Condirien : Enbinet 8. Bano. Tab. 85, Fig. 762. Argenville Soomorphofe. Tab. 8. Fig. 12.

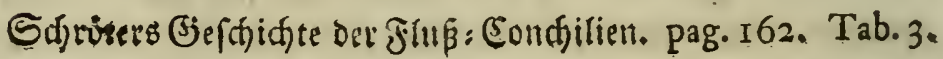
Fig. I,

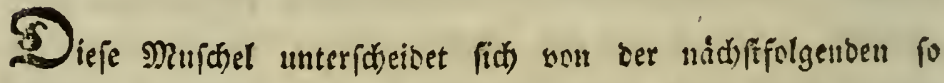
wenig, Daß fie einige Maturforid)er ferbft, fuir sine mo biejellese

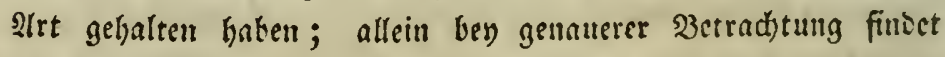
man fie mels verlángert, befonders bic vortere Eeite meft nus:

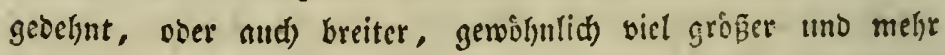
gensolbter, won Farbe melgr bunfelgrin, fie ift ferner atch

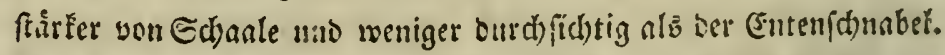

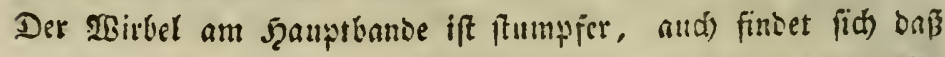
Der ohere Theil vor bem Edyloffe, Ia wo fich baz mujcuibfe 23anb hefinoet, cine mefre eingetrgenc Etelle, weld)e faft eintr rohmboibalija)en (Eindruct uad) vorne uns in ser sainge bitiet. Eie finbet fid in unfere biegend cinzig in scr echmutter, ott=

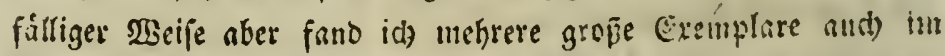

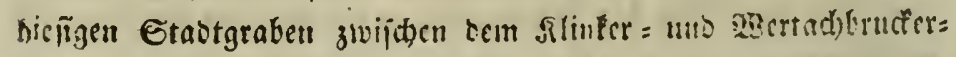

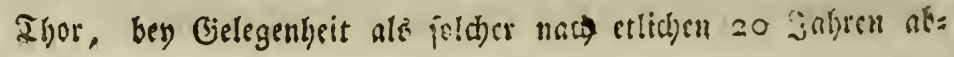




\section{$=7=$}

gelaffen utb gereinigt wurbe. Sie bat in ber fainge 2 yarifer 30lll unto 9 \&inicu, uno in ber Breite 5 3oll unt 9 Sinicn;

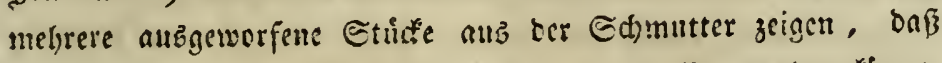
sicjer Mytillus aud) nod) viel gróper angetroffen werben bonte.

\section{Mytillus Anatinus. Linn. Der Entenifunater.}

M. testa ovali compressiuscula fragilissima margine membranaceo, natibus decorticatis.

Linn. Syst. Nat. pag. 3355 . Sp. 16.

Gualtieri Index Test. Conch. Tab. 7. Fig. E.

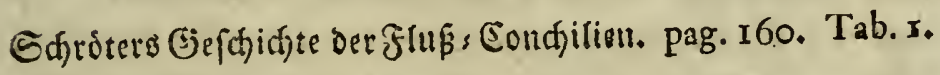

Fig. 2. 3.

Elemni; Condjilicn: Cabint. 8. ßand. pag. 189.. Tab. 86.

Fig 763 .

Stutm Dentfdlanis Fauna 6. গ(btheitumg. Die 2oùrmer. I. Şeft. Das 5. 6. unto 7. Situfer. Eine Felye artige 2lbbitbung voin ber Sdante und bem Servogner.

$\mathfrak{M}$

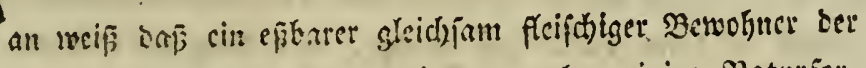
Echaale Den Enten zur Gpcije bient, woher einige Raturfor:

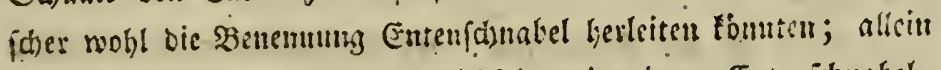

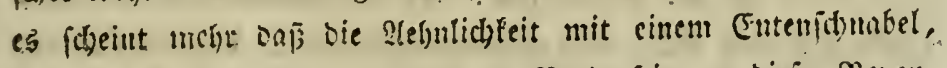

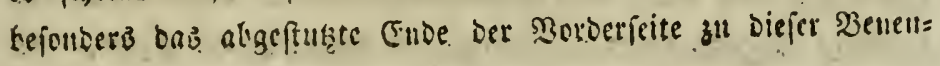




\section{$=8=$}

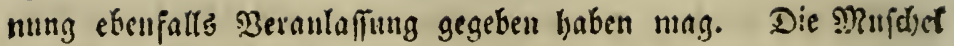
hat in sal Range you Sd)loffe biz-zum entgegengejetzten Rande 2 3oll uno 4 finien uno in oer Breite 4 3oll uno. 5 Simiea

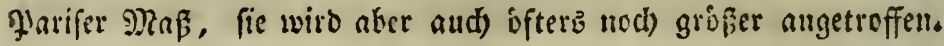

Eie bat cinc eyrunte Gefitalt, ift ctwas flad), hat eine

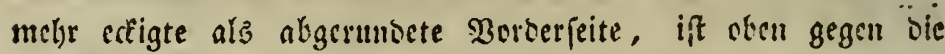
Mitte zufammengeoruclt und crlobly, gegen bie bintere Eeite

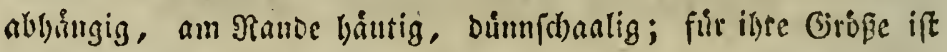

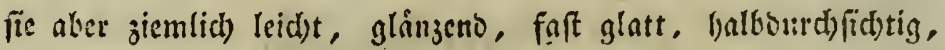

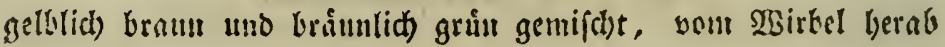
mit id)rig heroblatfenten Strablen geziert, nud') fintet man bogenformige Erreifan anf ifr, welde gegen ben গano bermu

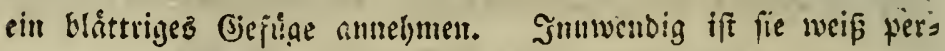
lenmutterartig ins Blátlid)e fpielent, uno mand)mall werten

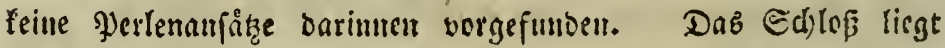
nad) ber. hintern afgermbeten Seite mb hat feine eingrcifmbe

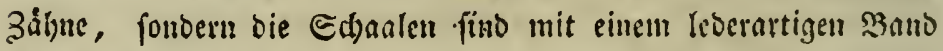
serfunden. Die SBirbelipitgen fiut flein, glatt, germuzelt, ctwas zugefpikgt nicht allemaly abgerieben, tefonters bey ben jungen (Exemplaren wie bey ber Mya pictorum, erfaciun

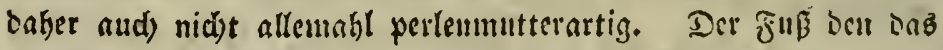
Zlier Sffterb 3 zoll ans ber Echaale heraubftrect", ift breit uno jugermbet, hat eine gelblid weife Farke, uno bient ihm in

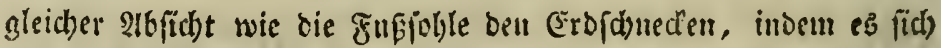
Damit an einer Etche feft anjegt, uno baum bie Ed)aale nads iid) zielts.

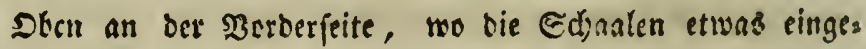

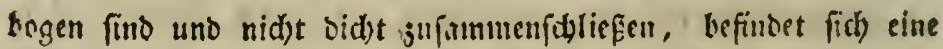

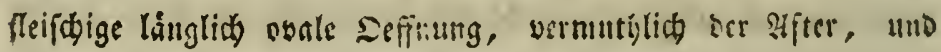




\section{$=9=$}

am Borterrande bie Franzen, we!dye mehrfach hinter cinanter

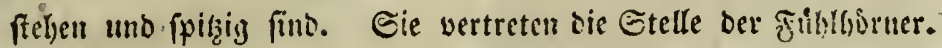

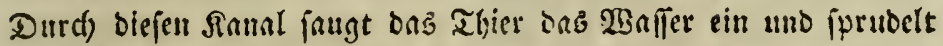

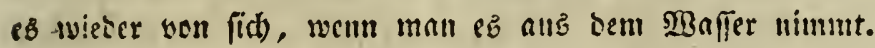

Shle 2tuffentlgalt fint bie Stritgrííen in und nußer ber Etabt, unb bann auch bie S(4)mutter.

\section{Bulla fontinalis. Linn. Die $\mathfrak{B a f f e r f i n f e}$}

B. testa ovata pellucida contraria: Spira obsoleta, apertura ovata oblonga.

Linn. Syst. Nat. pag. 3427. Sp. 18.

Edroters (jiefdidte der fflup = Condilien. Tab. 6. Fig. I6. a. b.

Der Naturforfdyer XV. Stude. Tab. I. Fig. I - 12. Egemniz Condilien: Eabinet 9. Sant. Tab. 103. Fig. 177.178.

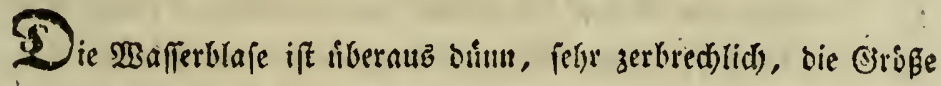
ift 3 Sarifer \&inien in Der Rannge uno 2 ginien in ber Breite, fie bat eine enformige beftalt, ift aufgellafen, von blafier gelblidber

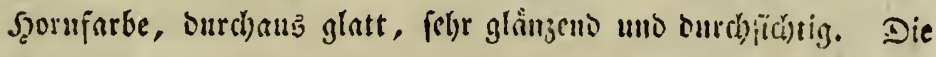
Munbsffuntug ift langlich), eyformig, grow, criveitert, uno berbut

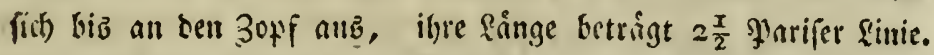
Die imere sippe an ier Epinofl ift etwas ankgebreitst unb bilbet 


\section{$=10=$}

einen (d)malen unb weiplid)en Ranb. Sic hat mefrentbeils 3 SBinoungen, bie erfere mad)t heynahe sen ganzen Uumfang ber Concbilic felbft anz, bie anoeren 2 Binoungen fino fefrs flein

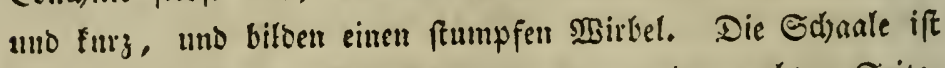
linfé gewumben und hat sie seffimung an ber red)ten Eeite. şsenn fid) bab Tljer nod) in ber edbanle befintet, fo lyat fie son bem ourdjidiumernden S3etwohner cine brisumlid) getbe Far:

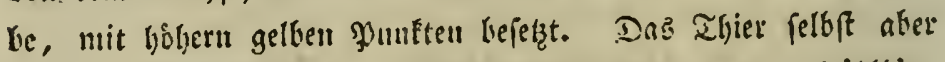

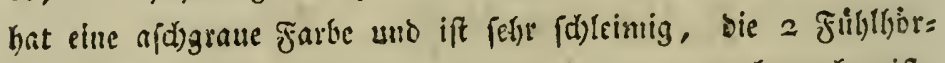

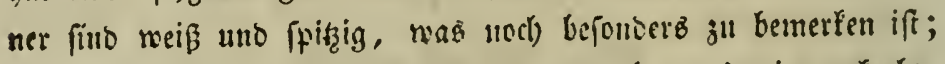
Dą Thier legt feinen Mautel in ectige Falten wic cine geferbte Şaut in Siried)en an ben Rânbern um Die Ed)nale hermm. Ser

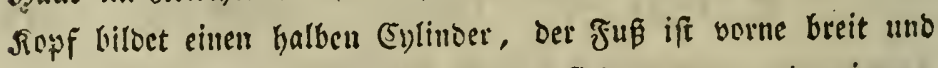
loinft hinten fpirzig zu. Die Shugen fiehet iman an ber iunetn

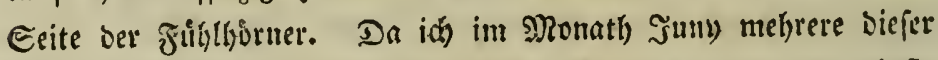

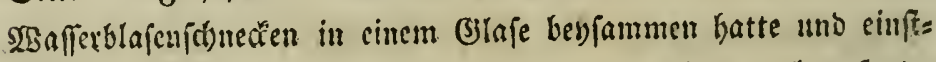
maly!b einige Ed)aalen unten am boben ganz rein und leer fant,

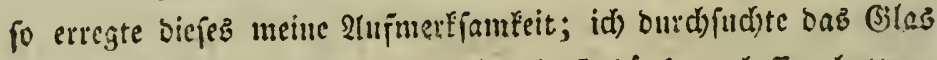
uno fant zwar bie Iljiere, weld)e bie Bielginfe verlaffen hatten,

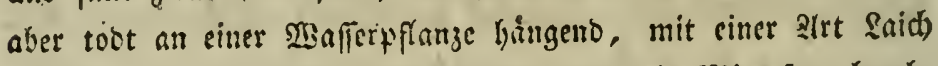
verfehen; ia id biefen abjomierte uno unter ein Microfop brach=

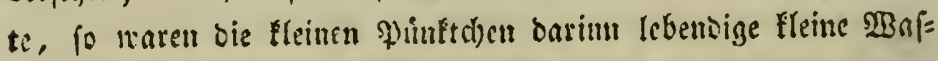

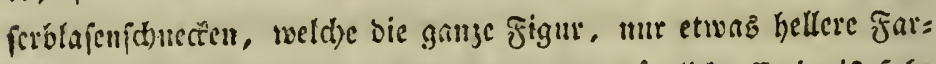
be, wie bie altoggenadjenen hattât. Das uimlidbe Ereignis fáxe ith bermach nodh an 6 (wemplaren, wohel) aher ber 3 croblyne:

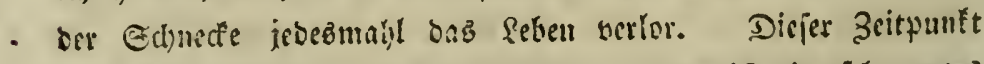

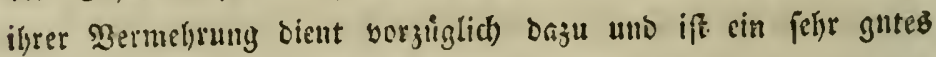
Mittel, Die Sod)anle rein fir cine Gammlung zul crifnlten ulo anjulliwatren; Deun es ift auserbem beynelye unoglid bab 


\section{$=I I=$}

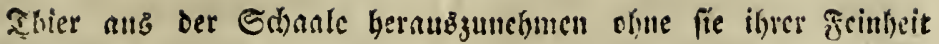

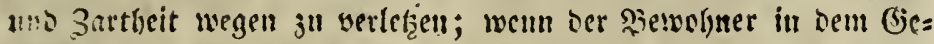
hifule sertroctuct, fo hleibt jebesmaht ein fdymirglid)er glect anf

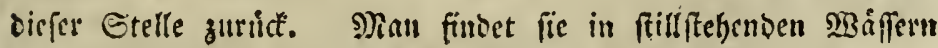

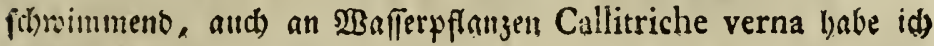
fie ifters angetroffer.

Sin Deutfdlans foll fie tiberaub felten feyn, in utrper

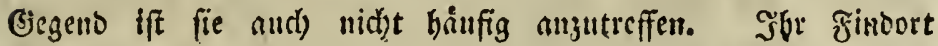

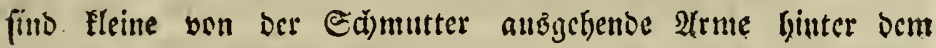
Sobel, wie aud binter fangwaip in Felegräben.

\section{Bulla hypnorum. Linn. Tab. I. Fig. 2. Die MRooblnfe.}

B. testa ovata pellucida contraria: fpira prominente apertura ovato lanceolata.

Linn. Syst. Nat. pag. 3428. Sp. 19.

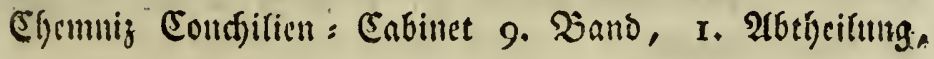
pag. 34, Tab. 103. Fig, 882, uno 883.

\$2 3 eil sie zeid)umug von Helix Achatina in Ed)roterg sfin :

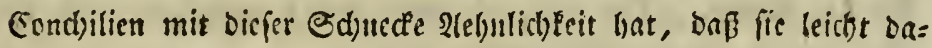
mit serwed)felt werten founte, mo weil Chemniz lange zmeifel= baft hen feincr Beftimmung wat, bis ibn jeine (d)webijd)en freun= be verifitert haben: onf es wirltidy sie wabre Bulla hypuorum 
(ev) uno bie zeid)unng nidht gant beutlid) vorgeffellt ift, fo glaus: be id) ocn Ptaturfremtoen baburd) gefolltig zu werben, wem id)

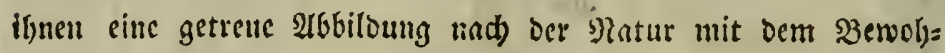
ner bier lifergebe.

- Die Brof̧e biefer Sd)anle ift 5 Plarifer finien finge (ber lezzte llmgang hat 3 finien uno bie librigen 4 haben 2 finien) unt ift in ber grospten siseite 2 finien. Cie ift thurmformig

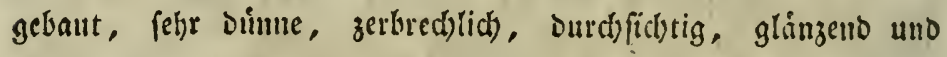
glatt, bod) zeigen fids suserorbentlid) feine Etrcifen, von gelts: braunlid)er farthe mo hat nuf monden Etcllen sfterz unregel:

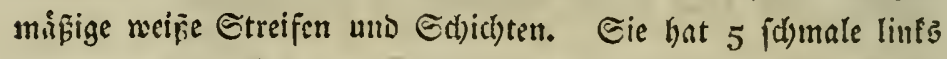

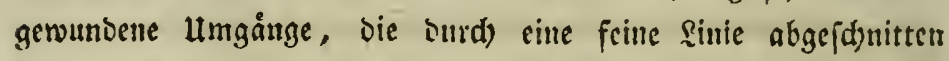
fint unt enbigen fid) in eine zientid) (d)arfe Epirze. Die Muts= offnung ift linglidbt owal uno verengert fich unterfialb. Der

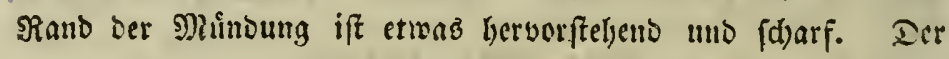

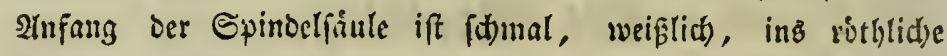
Fpielent, uno obne Falten. Die Єd)necte mad)t im ๔d)wimuren

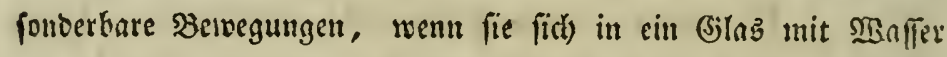
am Ranbe anfergt und anf etwos fioft, fo brebt fie bic Gajna= le bin unb her, und wenn fic ben fan ganz anzftrect, Daß bie Ed)anle madh unten gefelyrt ift, io idbifft fie wie ein Singn 'im $\mathfrak{B a f f e r}$ bertum.

Dab Thier felfit hat eine hlällich fanwarge Farbe uno bie

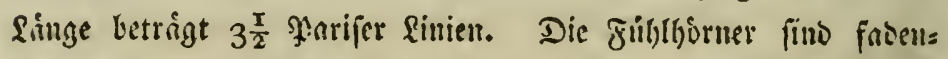
Formig, meiplich uno ipizig, an ben inueren Seiten befinben

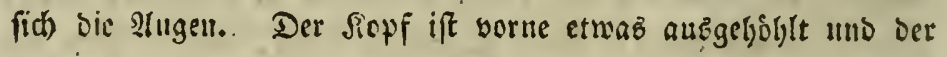
Jup gét in eine ftumpfe Epizze aus.

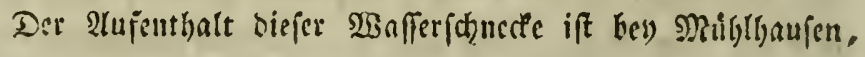

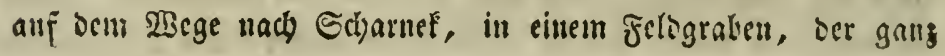




\section{$=13=$}

yon Marferpflanzen leer ift und einen thonigten fallammidaten

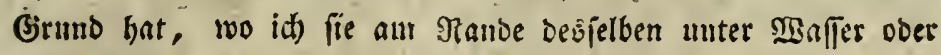
auf ber Dberflid)e fdiwimmento angetroffen hate.

\section{Turbo bidens. Linn. Die glatte Eroforaulfe.}

T. testa pellucida, anfractibus contrariis, futura fubcrenata, apertura posterius bidentata.

Linn. Syst. Nat. pag. 3609 . Sp. 87.

Gualtieri Index Teșt. Conch. Tab. 4. Fig. D. E. mit einer geferbten गath, ift Daber nidjt Turbo bidens, fonvern Turbo papillaris.

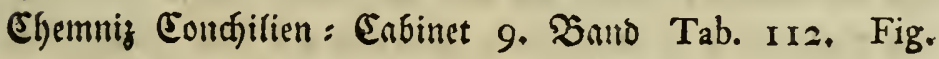
960. Nro. 1, 2.

Sturm Deutfalands Fauna 6. Aotheilung ber watrmer. 2. J̧eft bas 9. Siupfer.

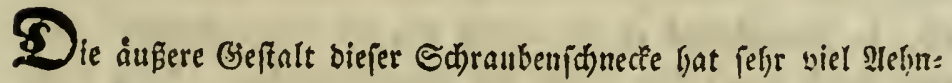
lid) keit mit sem Turbo perversus, allein bie volleomment llibereinftimmung verfdrinbet, fobald man beyde (jel)ăufe ge= nau mit einanber vergleidhet; and) verfichert uns ber Şerr fion: ferenz = Rath Miller, Daß fich nie linbe mit einanber begat:

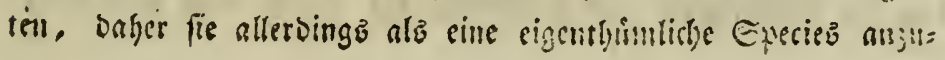




\section{$=14 \Rightarrow$}

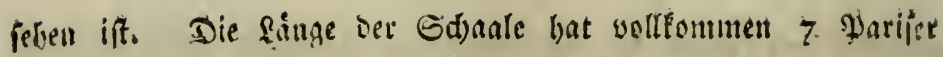

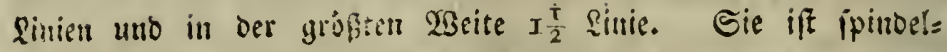
formig gebaut, bie Girmojarbe ift rothlid) (renun; burd)fidstig,

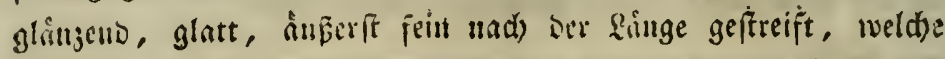
Etreifen man an ber litgten Srifitoung beutlider bemerfen fant. Sie bat to lintiggertebte SBindungen, weld)e gegen bie Nitte nujgebhajen uno burd) éinen glatten Einjojinitt unterjdieben füb.

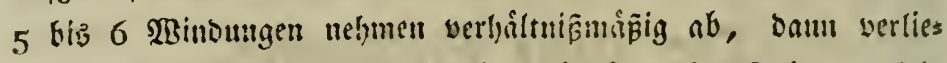
sell fich bie tubrigen (d)ull in elute abgeitumpfte Epize. Die

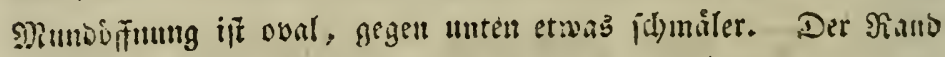

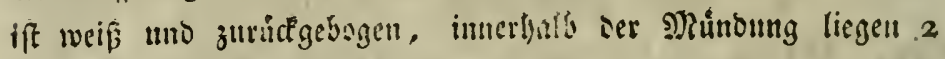

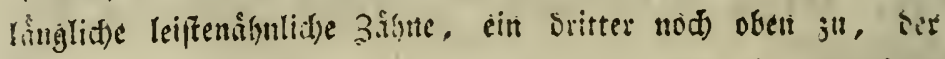
nber tief im Sdlume liegrt; ber mittlere 3abn iff ber gripifte

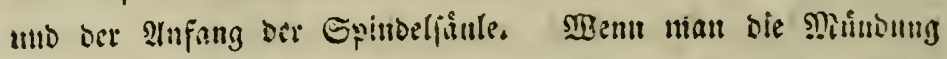
nhlurid), fo Eanu man ben obern 3ulyn heraubuthmen; man hes merft nlbaban nod) cinige oergleiden Faltenleiften. Sie bat

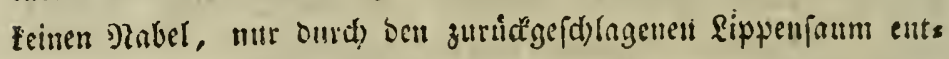

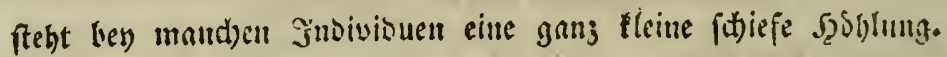

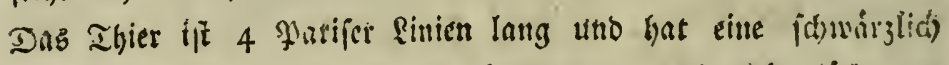
graue Farbe, Die Fupfolyle ift aber beller. Die Deferflide orr

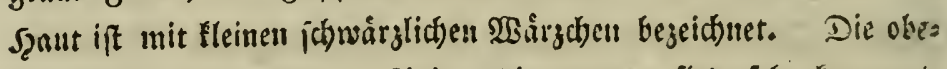

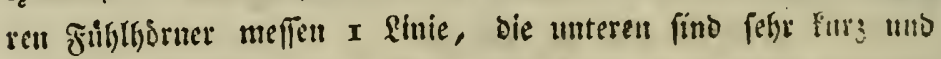
ahgeftumpft. SBenn bab (behånfe nod) vou bem Thier bewolynt ift, fo unteridelibet es fid) wentig vou ber leeren Ed)anle, alz blo B burdh eine bellere Farbe uns Durdjfid)tigfeit. Nian trifft zuweilen, Gefrinfe nod) mit ben Ihiere an, welde gegen bie

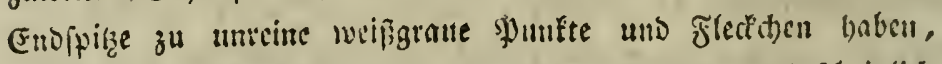

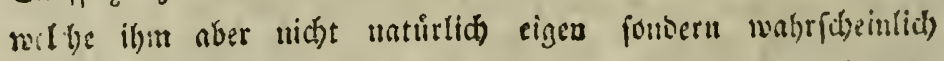

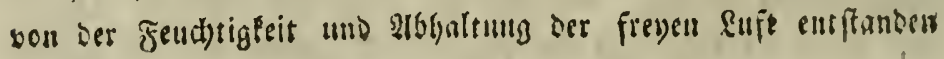




\section{$=15=$}

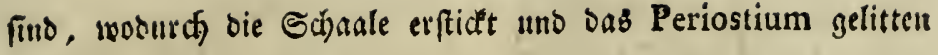
lyaben mag.

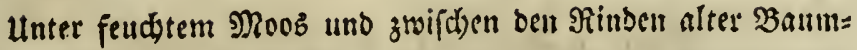

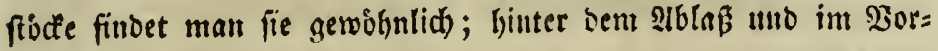

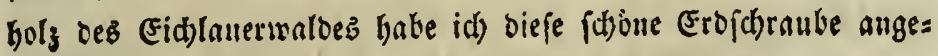
troffen, welde in biefiger Begento nid)t gemein iff. 


\section{$=16=$}

\section{Turbo perversus. Linn.}

\section{Die geftrifte Linfoldyrauter.}

T. testa pellucida: anfractibus contrariis non crenatis apertura tridentata.

Linn. Syst. Nat. pag. 3609. Sp. 88.

Gdyrders AGhandrung lifer bie Ero: Condjilien un Than:

gelfedt Tab. I. Fig. 4 .

Efemniz Conffilien : Eabinet 9. Samb, pag. I16. Tab. I12. Fig. 959. a unt b. Eine unridtige sidv: biloung.

Siflutterberf Acta Helvetica Vol, 5. Tab. 3. A. Fig.

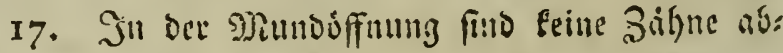
gebildet Fig. 18. i ît mit Desm ₹ijier gejectfuet.

Gualtieri Index Test. Conch. Tab. 4. Fig. C. Die aritis: mung gleidet melge ber glatten Erofdranbe Turbo bidens.

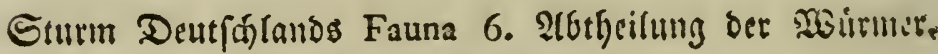
2. Şeft Das IO. Silupfer.

\section{(5)}

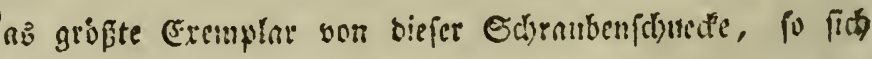

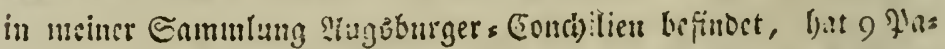
rifer finien in oer Range mo in Der slsteite, wo fie nu Diffifen

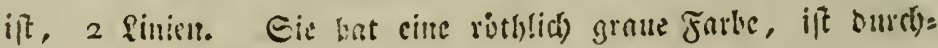

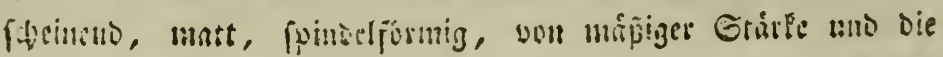
sbit: 


\section{$=17=$}

Sberflidje wiro mit borijontalen erhabenen Etreifen ganz tauly

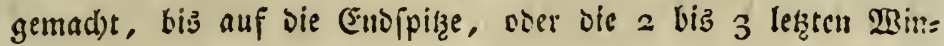
iungen, milde lyornartig, burchjidtig und glatt fint. Eie bat 13 finfégange, oft eud weniger, Dic aber nidyt verbifts

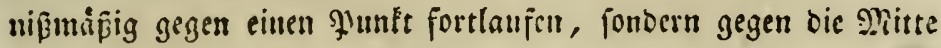
etwas baudbig fins uno fid) in cine ftumpfe Epizze endigen. Die Munbofinnung if owal, oben edig, unterbalb screngt jie fiid) amb ifi ilgrer finge nad) etwab zufammengedrict. Dit.

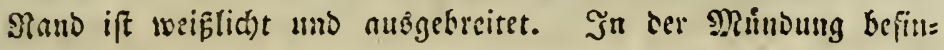

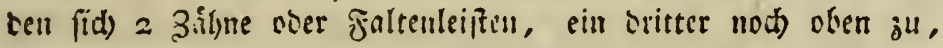
eer aber fo tief im Ed)lunde liegt, sap man ign fram bemer:

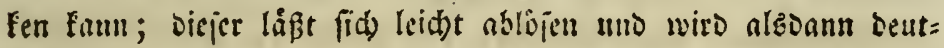

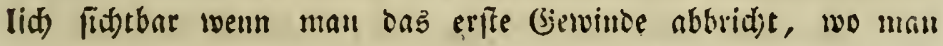
alsbann nod) einige berglcid)en faltenleifteu bemerfen fant. Das Iflict fieltt Turbo bidens ganj gleid) uno ift aud) van Derjąlben Brópie.

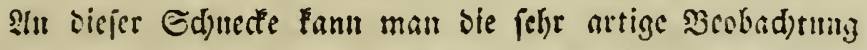
ma(t)en, wie jid) nad) uno nad) Bermebrungen ber Ulmginnge

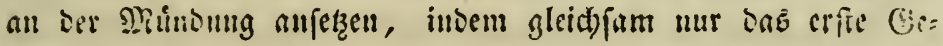

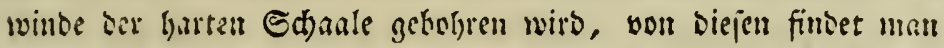

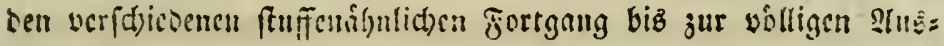
bilbung ber ganjen Ed)nte. Dieje Speciez ift fehr gemcin,

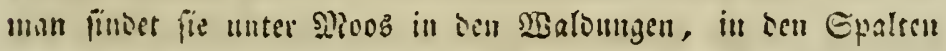

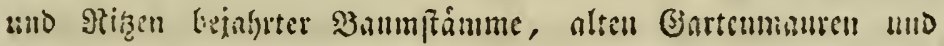
anteren atgutitgen sorter. 


\section{$=18=$}

\section{Turbo conversus. Mihi. Tab. 2. Fig. 3.}

\section{Die Eleine lineżgebrefte Erofintaute.}

8

roh aller angemanden Mlibe habe idf leine Defdreibung ge: funben, weldbe biefe recht attige Ed)medte darafterifint hitte;

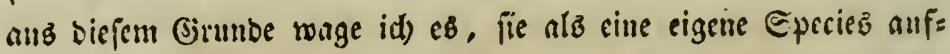

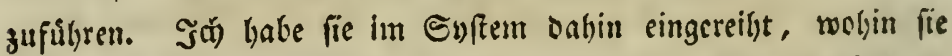

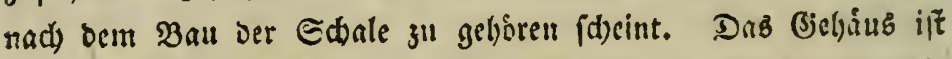
5 Plarijer Rinien lang unD in ber grofften Breite $x \frac{x}{4}$ Sinie. Eie

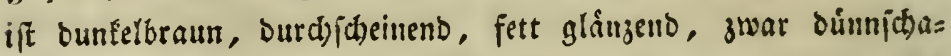
lig, aber bod) nidst leid)t zerbleálid, fpindelformig uno bie Länge berab mit regelmåpigen erbatienen Etreifen bejcidinct; bis auf rie Entipize ober bie $2-3$ Icḩten 2 Bindungen, weldse bornartig uno glatt fino. In ibrer aubern Bilbung bat fie visl 2(ehnlid)feit mit Turbo perversus, nnterfdeibet fid aber von Demrelben burd) ibre Farbe, mindore (jirīe, wie aud burd) Die Faltenleiften in oer Sglunoung mo ourd) bie Mundiffum

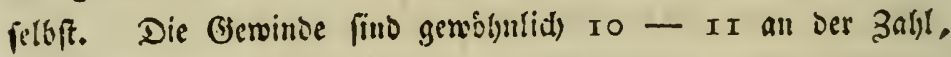

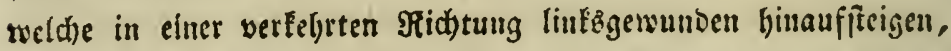
in Der SRitte etwas baudbig finb uno in cine ftumpfe Epitge aubigeben. Die Manobffunng ift oval, unterbalb verengt uno an ber Gpindallefze bemerft man 5 mandamal 6 Faltenleiften, woson bie obere uno untere großßer unb bervorftebeno fino. Der

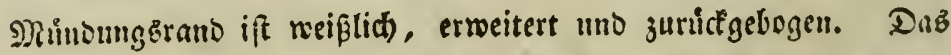
Lbier lietrigt in ber fänge $2 \frac{\mathrm{T}}{2}$ Parifer linien; sie oberet

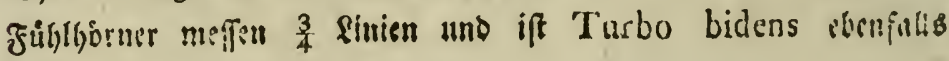
\&ดulic). 


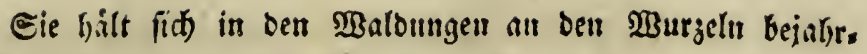
ter (Eid)en = und Budj/finme unter $\mathfrak{R o o s}$ auf und wird nur iparjam angetroffen.

\section{Turbo quadridens. Geoffroy.}

\section{Die biergafanige Eroffaraube.}

T. testa sinistrorsa flavicante: apertura quadridentata.

Linn. Syst. Nat. pag. 3610. Sp. 92.

Lister Sinopsis Tab. 40. Fig. 38.

Ehemniz Condbilien: Cabinet 9. Band, I. 26theilung, pag. 123, Tab. I 12, Fig. 965.

(s)

g ift auffalletio, baß bie vornebmften Condiliologen, als Ehemuis, Miller und andere, in ifren Bejd)reibungent bicje Eris

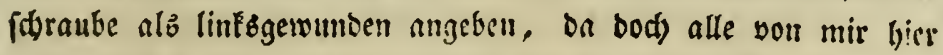

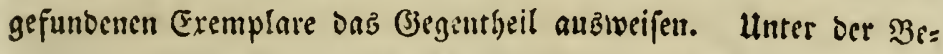

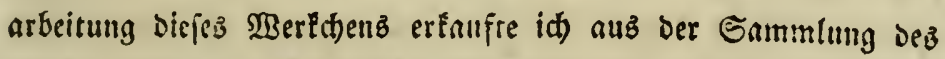

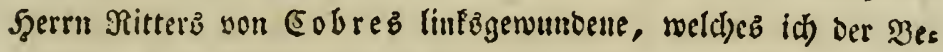
merkung bir fuir pelfr werth balte.

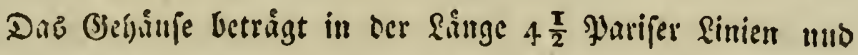
in oer 23 reite $1 \frac{3}{4}$ Sinien, fie ift mells fegel = alb cylinberfor: mig, hellbriunlid), ber sainge bernb fein geftreift, wenig glins

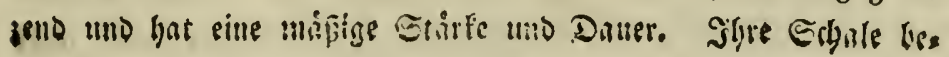




\section{$=20=$}

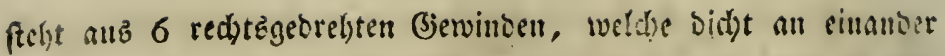

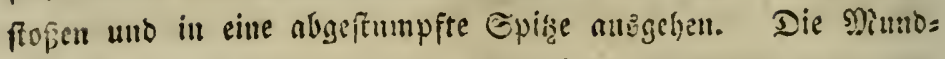
offunng ift halb oval mit 4 weifen 3ingenen verferhen, moson un= ten einer in ber פucre liegt, biuter biejem ciu jwenter, am Gei= temranbe bet ămfern Rippe ein britter uno gegemiberftehent nad) oben ein vierter fich befibdet (wem man nimlid bie Ed)ale fo

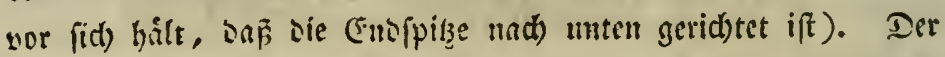

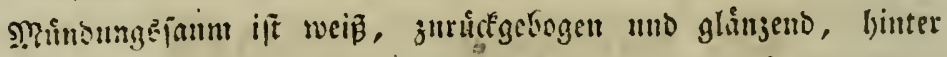

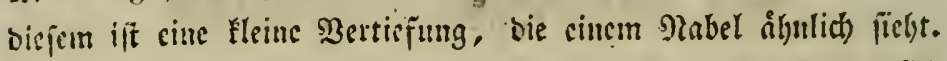

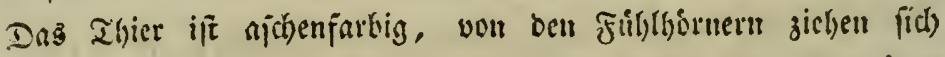

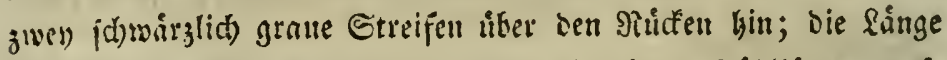

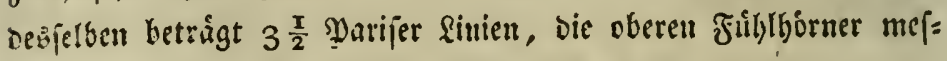
ien $\frac{3}{4}$ Rinie.

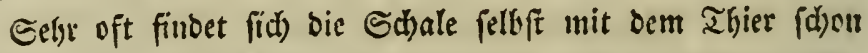
gan; serbleid)t, gleidj) am wie abgeftorben uno basourd) geflect't,

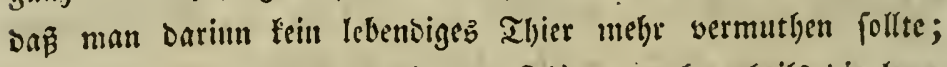
man finoet fie nur felten mit bem 2 bicre, mefrentheilo ble leere verwitterte Edale. Sie wohut all Seligeftn mo bergid)ten (je) genten, zwifden nieberen \$flnujen uno Mioos, in Befellidjaft

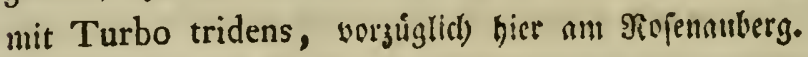




\section{Turbo tridens. Miller. (Octo den- tata. Stroter.)}

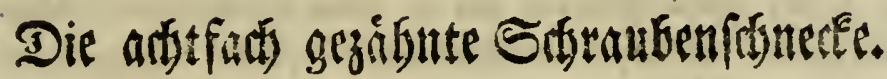

\section{T. testa albida: apertura tridentata.}

Linn. Syst. Nat. pag. 36r r. Sp. 93.

Gualtieri Index Test. Conch. Tab. 4. Fig. F. Diefe 3eidjnung bat mehr Iflynlid)eeit mit Turbo quadridens, in Der Munbofinumg fiegt man mur einen Zahn.

Edjoters Ero: Condilien un Thangelftedt, pag. 140. Tab. I. Fig. 6, Enmftlos geftoden.

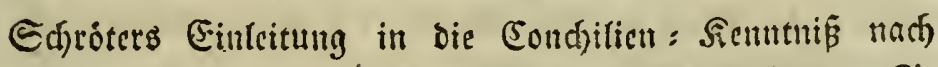
Linné 2. Banno, pag. 59. Diefe beydon Citata feflen in Linn. System.

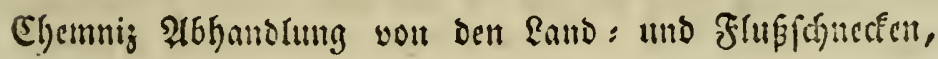
pag. 167. Tab. 135. Fig. I236.

913

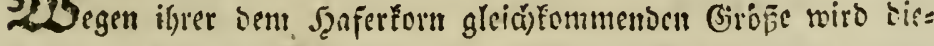

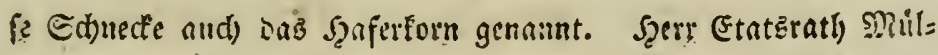

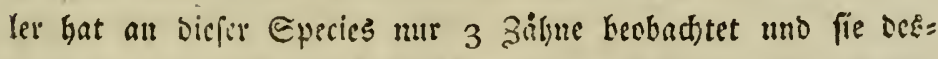
wegen mit Deut Nalymen Turbo tridens belegt. Şerr Nyaffor

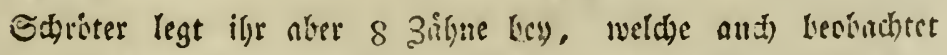
merben fonnen. Die Linge sicfer thimen Erofdrante if 3 glas rifer sinien und sic Breite I Sinte; fie iff surdjpictig, fits: firu 


\section{$=22=$}

gefireift, menig glánzeno, etwab fegelformig unb hat eine bráuns lidje Sernfarthe. Mat beun lebendigen ahier ift fie am 3opf

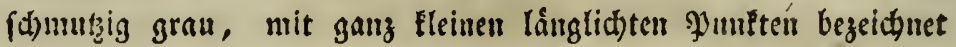
unb an ber erften SBintung bat fie cinen gelblid)braunen láng: liden flect. Sie bat 8 red)tzgebrefte $\mathfrak{B}$ Bindungen, weld)e burd" einen Fleinen (Einfibuitt getrenut fino uno fid) in eine ftumpfe Spike enoigen. Die Nimboffung ift balb oual uno mit 8

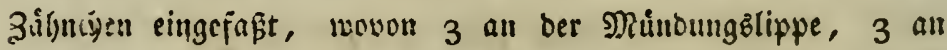
oer Spintellejze, weldye aber etwaz fleiner fino, unten nad oufien eincr, weld)er ganz voine liegt, linter biefem feitwårts iff nod) ein guneyter befinolid). Der áabere Nano ift ein wentg zutridgelogen, mit einer weisen Einfaffung, linter meld)er von auß̧en einige meife furze Stridbe zu bemerken fint. Sie hat

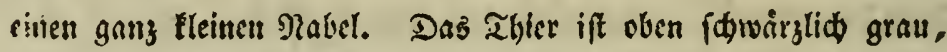

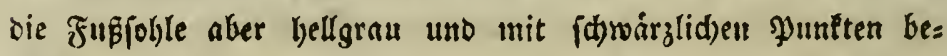
jeidunet, bie långe bešfrlten betrågt $\times \frac{3}{4}$ yjarifer Rinien, bie sberen fuighborner meffen I sinie.

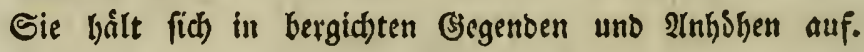
Bey. 2lugbburg finbet fie fid an bem nahe liegeuten Rofenau= berge. 


\section{$=23=$}

\section{Turbo Muscorum. Linn.}

\section{Die Moogfiteraube.}

T. testa ovata obtusa pellucida: anfractibus fenis fecundis, apertura edentula.

Linn. Syst. Nat. pag. $36 \mathrm{I}$. Sp. 94.

Lister Hist. Anim. Angl. Tab. 2. Fig. 6.

Shroters (5ro: Conhilien um Thangelftedt, pag. I40.

Tab. I. Fig 7. unrid)tig und Eunftos geftod)ert.

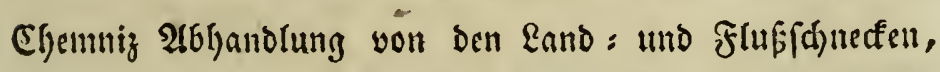
pag. 6r. Tab. 123. Fig. 1076. Nro. I IIID 2 .

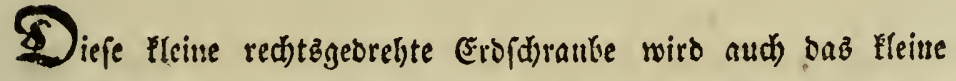

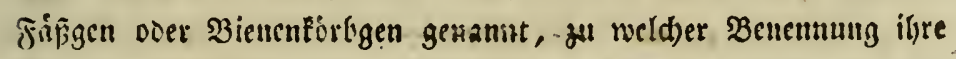
Form unto Biloung Beranlafiung gegelen lat. B̧enn dieje

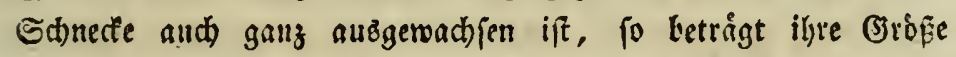
Dod) nid)t megr alz $x \frac{3}{4}$ P̧arifer Sinien in ber fänge unt $\frac{3}{4}$ ? $i=$

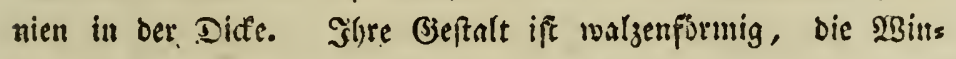
Dungen fino rlle gleich unb bnudbig, ausgenommen bie lefzteren, weld)e fith ftumpf entigen, ooer gleid) am abgerunbet fint. Die

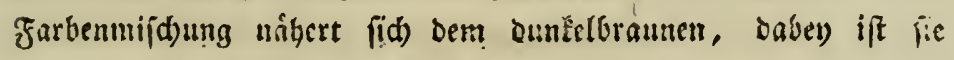
ferner fein gefercift, duted)fid)tig, felgr binu, zait uno glatt.

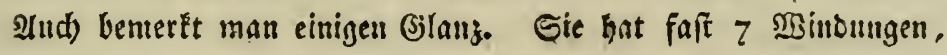

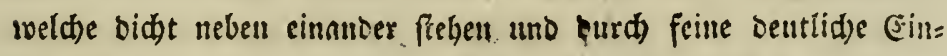
id)nitte von cinander getrennt finb. Die Mtundifinumg ift balk

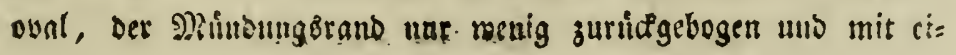




\section{$=24=$}

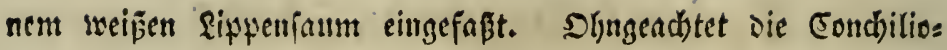
logen an ihe feinen $3 a \mathfrak{h}$ w wollen beobad)tet haben, fo wito man boch) unten in ber Mitte ocr Munbeffuunz eine Spur ober eis nen Intrang einer zalnformigen Erhabenlyeit gewalyr. Sie bat

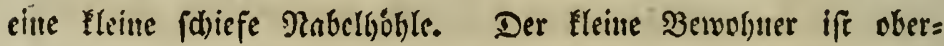

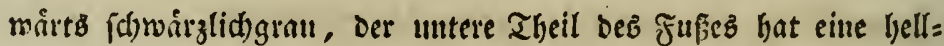

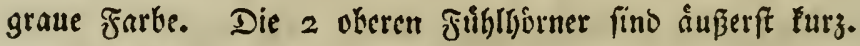

Nan finbet fie am Rofennuterg wie and) am Hier uno

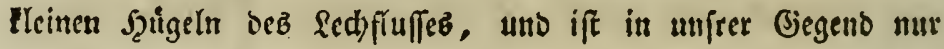
fparjam anzutreffen. 


\section{$=25=$ \\ Helix Lapicida. Linn. \\ Der Steimpiretr.}

H. testa umbilicata utrinque convexa: apertura transversali marginata ovata. Linn. Syst. Nat. pag. 3613. Sp. 2.

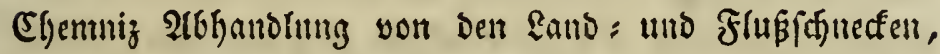
Tab. 126. Fig. Iro7. pag. 88.

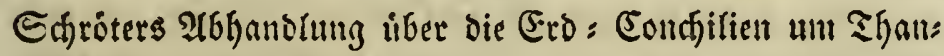
gelfeot, Fig. 23.

Eturm Deutfiflands Fauna 6, 2G6theiltung. Die 2 Silts mer. 2. Szeft, Das 5. Siluper.

Der Mitter you Linné hat biefe Sdfriedfe beỏmegen ben Stcin= picter genannt, weil fie sermógeno fenn foll, Den Falfiftin ju zernngen, weld)ez aber bic mefrffen Condhiliologen bejwcifeln unllen; id bate mebrere Berfude mit biejer Helix angeficllt. aler an berjelben bieje (5igenjd)aft aud) nid)t wahrnelgmen fun:

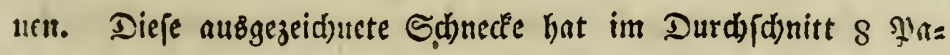
rifer finien und 3 sinter in ber Şobe, fie ift vout foldor feid)=

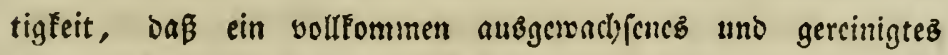

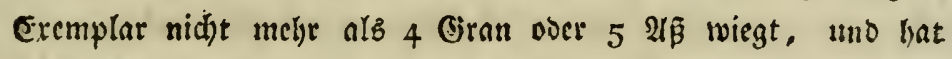
bakey eine zicmlide Feffigfeit uno Dautr, woju wastrfücinlich ing fonberbahter $\mathfrak{B a n}$ vicles begtragen mag. "Die áuberlide Bieftalt ift oben uno unten etwaz gewollt, uno ifs Silfen

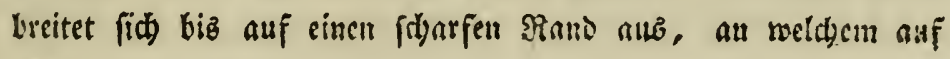




\section{$=26=$}

beyben Seiten ein ja)malez braunes $\mathfrak{B a n b}$ berumläuft; fie bat einen (d)wad)en Fettglin\}, ift hornartig uno surd)fidtig, unb mit einer Şansluppe betrad)tet, ift fie mit erhabenen Sylints:

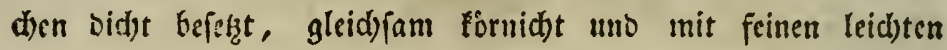
Suerrunzeln burdjzogen. Sie hat $5 \frac{\mathrm{T}}{2}$ bid)t aneinnnoer nieber= gebricte 2 Bindoungen, weld)e eine roftgelflid)e Farbe baken uno Durch braumrthe Flecten wellenformig marmorint fint. Die obale S) Muroffnung liegt in ber Durere, ber untere Ecitemrano ift auz= getehnt, oberwairts aber zuridegebogen unb unit einem fdónen wei Ben Rano umgeben, weld)er nach) innen glatt unb glänzent ift. Der Mabel ift offen uno geft biš zur Endipize, in mel= der man aber nidyt alle Sisindungen bcutlid) bemerten kann.

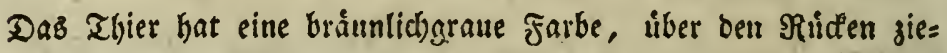
hen fid) 2 dunfle Etreifen jegen ben Mantel hin, bie Şals= baut ift wie dagrinirt, ber bintere Theil bez Juß̋ez, ber eine hellere Farbe hat, gelyt in eine jiemlid) (d)arfe Epiţe altb. Die Farben von biefer Sd)necte weiden untereinander etwas ab, ins Dem bey mandyen Indivionen foldye viel bunfler ( fino, woran wabrid)einlid) bie Ber\{fjebenlyeit bez alterz urface

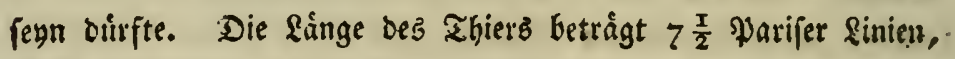
Die grope Fubllyouner mefien 3 Sinien.

S̈n unirer G̈egend ift bieje Speciez felten, man findet, fie

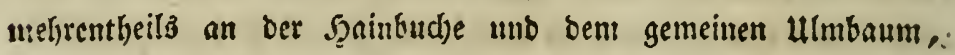

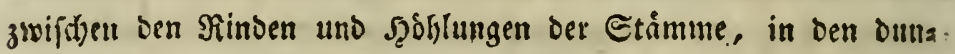

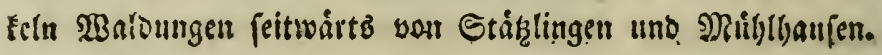




\section{Helix incarnata. Schroeter.}

\section{Die fleiføfarbige Sthnecte.}

H. testa perforata fubglobosa fubcarinata cornea: anfractibus fex, labro incarnato.

Linn. Syst. Nat. pag. 3617. Sp. 17.

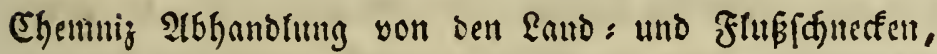
Tab. 133. Fig. 1206.

Sd)roters A6handlung úber bie Ero : Condilien um Than: gelfedt, pag. 174. Nro. 52-54. Tab. 2. Fig. I8.

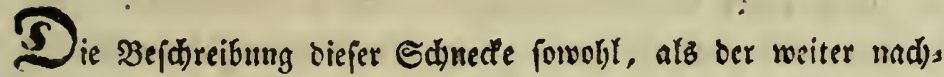
forgenocu Helix Isognomostomos muß Gmelin nidbt geforig. sufgefap̧t lyaben, fonft batte er biefelbe, on fie ilgrer Erructur: nat) nidjts weniger als ifjarf geranoet ift, bey ber umarbeis:

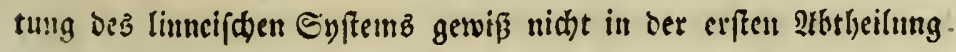
felen laffen, fontern vietullyr in bie zwente eingereiht, werhin fie aud) cigentlid bevoe geboren; ba id) aber biefez Syftem cins maly! als (jirumolage angenommen hate, fo fann nuch bicrimu

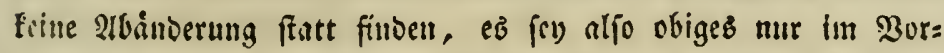

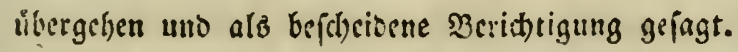

Die Edale hallt im Durdmeffice vollfommen $\frac{T}{2}$ 3oll unto

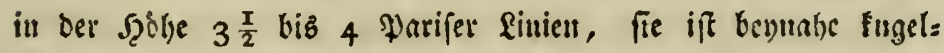
formig, in ber Duere fein geftreift, glingent, jientict) ourd)= fitbrig uno yon mípiger Etirle. Gewobnlid if fie blậgram, 


\section{$=28=$}

jebod) finter man fie, wicwolgl felten, ganz weiñ, pertenmutter,

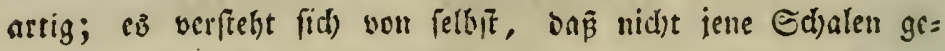
mennt werben, meldje ourd) গiegen und enne meip getleid)t ober verwittert fino, wie fich Diefez bem forfher oft bartictet. sin Der grofen 2 Sintung wiro man eine Spur einet fomalcn

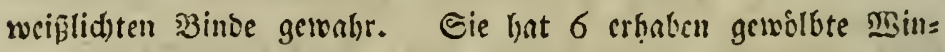
bungen, wobon bie erfîe grop uno band)igt ift, bie tibrigan uch)= men verhiltnifmåsig ab uno enbigen fid) in eine abgeftumpite Enofpikge. Die Monbiffnung ift halbmonbformig, ber Rant

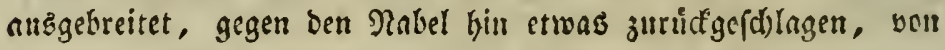

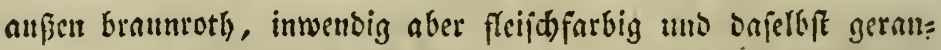

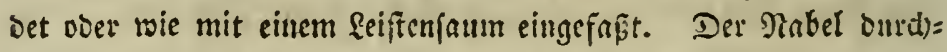
bohrt zwar dab ganze beholufe, afer er ift fo eng, daś man in bempelben bie $\mathfrak{x}$ Bintonngen nidht anterfdyeiben faun.

Daz Ihier ift oben fdusirglid, unten afdenfarbig. Die

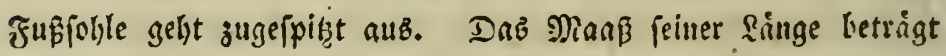

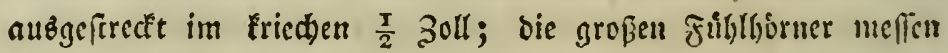
vollfommen 2 Yarifer sinien. Bey bem feben bes Thiers ift bie Ed)ale mit (d)warglidsen flecten uno Juntten bejprengt, sie ihr ein jierlid)es Sinfergen geben.

In fendten fdiattigten sorten unter Gieftraiudje, weldje

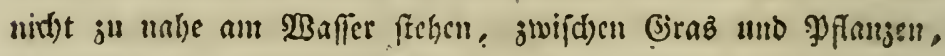
wiro man sicje Ed)mede. nidjt felten antreffent. 


\section{$=29=$}

\section{Helix planorbis. Linn.}

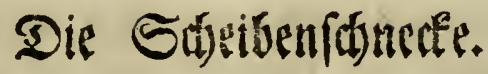

$\mathrm{H}$ : testa fubcarinata umbilicata plana fupra concava: apertura oblique ovata utrinque acuta.

Linn. Syst. Nat. pag. 3617. Sp. 20.

Gualtieri Index Teft. Conch. Tab. 4. Fig. E. E.

Sdjwammerdam Bibel Der Siatur, Tab. 10. Fig. 5. Petiveri Opera Hist. Nat. Spect. London 1767. Tab. 100. Fig. 14. C. 216.

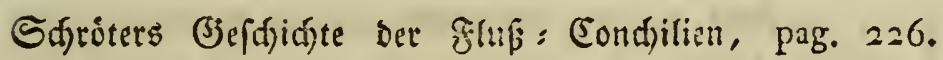
Tab. 5. Fig. I3,

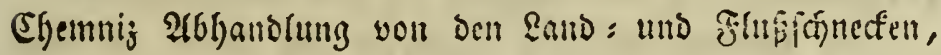
pag. 84. Tab. I26. Fig. I102. Nro. I Hild 2.

\section{8}




\section{$=30=$}

puntt verminderu uno onjelbit auf beyben Eeiten eine fleine Bertiejung haben.

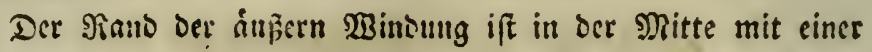
weipiliden idarfen sante cingefaßt, bie wie gefaljt zu fesun fheint. Die Nundoffinung ift vou einer ovalen Gefta!t und hat 2 Ecten ober Bintel, Der obere SRünoungärand ftelgt eine linie weitel vor.

Dab Thier bat in ber finge 3 yुarifer finieth, eine fdwarz ze Farbe uno if mit 2 rothen fabcuformigen Futhlfornern serfes ben, an Deren inmern Eeite fid) bie Angen befintocu. Oeegen oab sidht gehalten, fdeint ber Bervorner beutlid) burd, ser

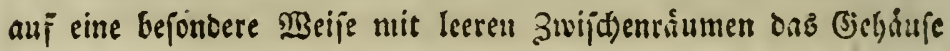
aแริfน์llt.

MBem man bie lebendige Sdjnede mit einer Tabel ourd)= ftid)t, fo fliept aus ber verwunbeten Stelle eine purpurrothe

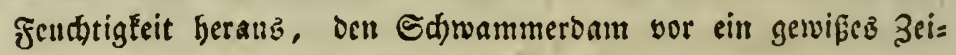
den auniumt, Daß́ bicjer Sd)nede ihs $\mathfrak{B}$ lut purpurroth fene.

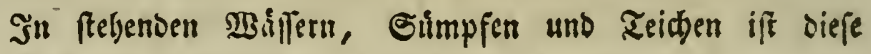
Sdurecte fegr gemein. 


\section{$=3 \mathrm{I}=$}

\section{Helix complanata. Linn.}

\section{Dả platte 2 (mmonę)}

\section{H. testa deorsum carinata umbilicata}

convexa fubtus plana: apertura femicordata.

Liun. Syst. Nat. pag. 3617. Sp. 21.

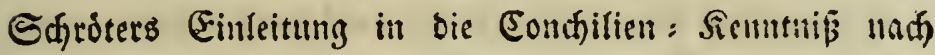
Linn. 2. Bamb, pag. I29. Tab. 4. Fig. I.

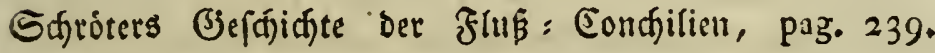
Tah. 5. Fig. $22-25$. 'Tab. min. C.

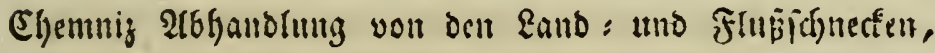
pag. 96. Tab. 127. Fig. I121 - 1123 .

Sdimammerdaun ßibel Der Natur; pag. 80. Tab. 10. Fig. 3. 4. Mit bem Slier.

⿷5

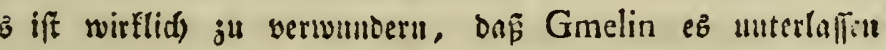
bat, lidh in jeinem Linn. Syst. Nat. auj oie svolfgetathene nad) Der statur gejeidurte arbsiloming siejer Edyuede it Edhivammerdams Bibel Der Natur z" bermjen.

Dtto Friebrid) Mhiller uno Sd)robter baben bicfe 3eidbnung fuir Helix Cornea angegeben und fid bantit ganj anffalleno

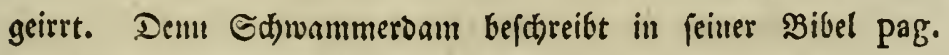
80. eine platte Sd)necfe nady ber slbbiloung Tab. Io. Fig. 3. bie platte Ed)ucte (Helix complanata) fulgenbermaffen: „Dab

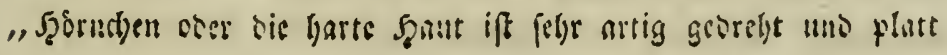




\section{$=32=$}

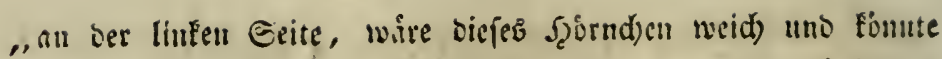

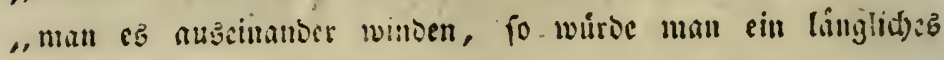

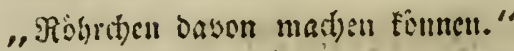

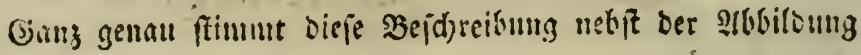
mit mimen vor mil liegenien Exemplare tiberein.

siljsm nun Ed)wammerbam glciá) Darauf fortfábrt in fri=

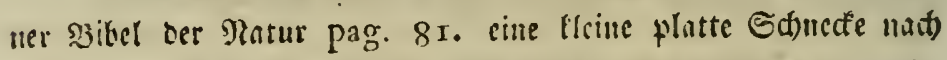
zer 9tsfildumg Tab. 10. Fig. 5. Folgand zu kejdreiten: "Dies

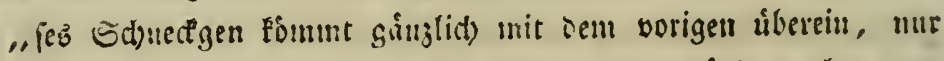

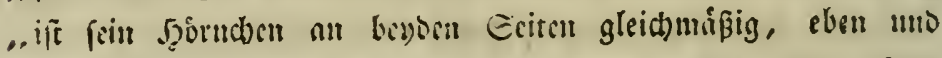

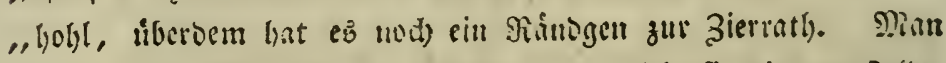

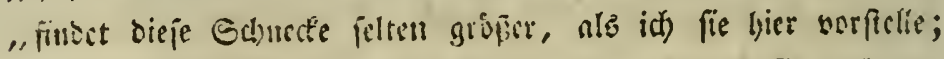

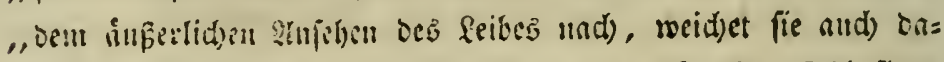

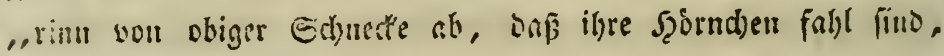

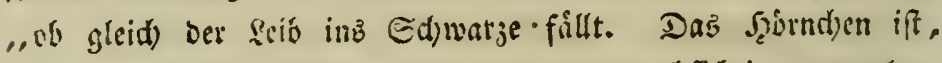

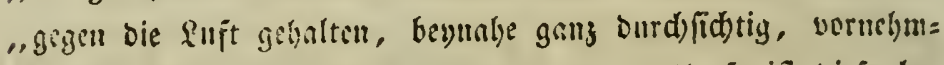

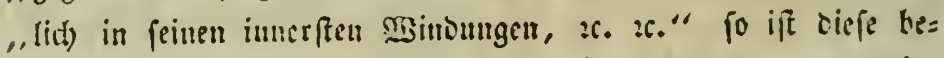
frimmt feime andere, als bic wor mir lebendig liegente Helix

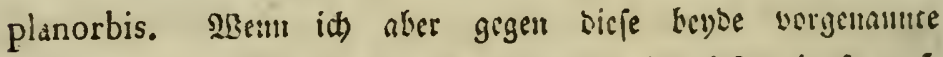
Eduncten bie Helix cornea vergleide, fo ift bicies cin fo anf=

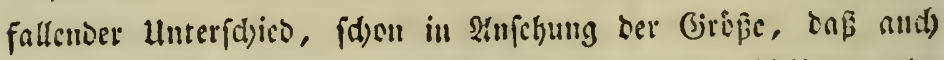

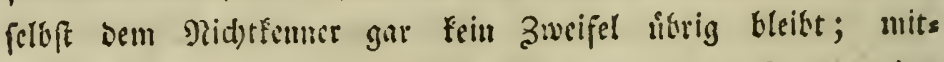
bin ganj entfd)icben waly, baß son erfthenaunten Stutoren Der

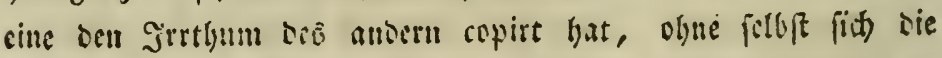

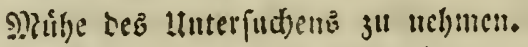

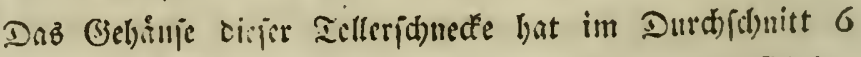

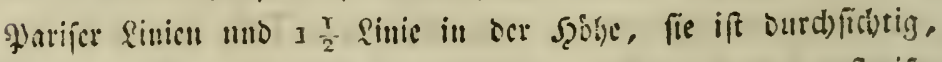

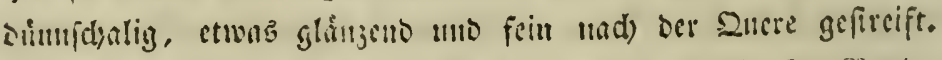

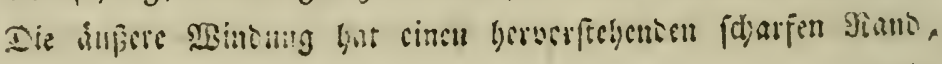




\section{$=33=$}

gerabe an ber untern Fli̊he, woburd) bie untere Seite beb (Bis= winder gans platt wito. Won ber obern Seite finn biejer Rnno

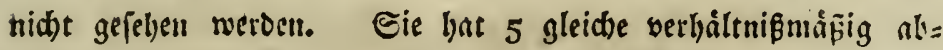
nebmente Ssinbungen, bie fidh um fidh felbft berumzielyeu, wels de auf ber obern Ceite gersolbt, unten aber platt finb.

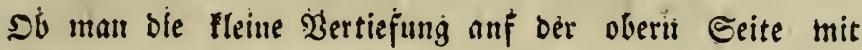
einem Nabel vergleidsen fonne, laffe id) oabiut geffellt pesnn.

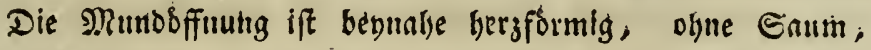
etwas ectig, sie obere sippe ifit merfilid) vorgezogen. Eie bringt ben nåmlid)en purpurrothen Ed)leim berbor, wie Helix planorbis.

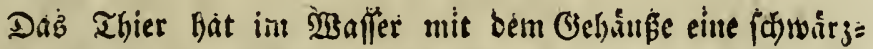
lidse, aber bie leere Edjale cine bráunlidgelbe farbe, mian miip fie abet von ifrem Ed)mutz reinigen, wenn man ilyr the tưrlides farbenfleib felgen mill.

Dab Thier felbfi ift Helix planorbís att farthe uno

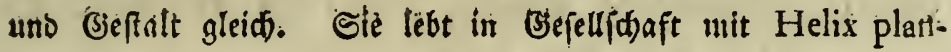
orbis, an meld)en Drten man (ié zugleid) artreffert fann, aul) ift lie wie bie sorige fefre gemein, aber vollitůndig auşgrs wadjene Exemplarient fins nid)t fo bảufig anzatreffen. 


$$
=34=
$$

\section{Helix vortex. Linn. Die wirterfderibe.}

H. testa fupra concava: apertura ovali plana.

$$
\text { Linn. Syst. pag. } 3620 . \text { Sp. } 30 .
$$

Petiveri Opara Hist. Nat. Spect. London 1767. Tab. 92. Fig. 6.

Lister Hist. Animal. Angl. Tab. 2. Fig. 28.

Gualtieri Index Test. Conchil. Tab. 4. Fig. G G.

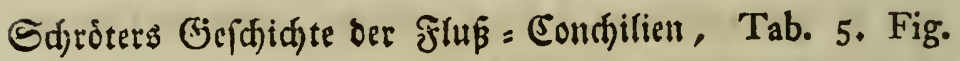
I6. 17 .

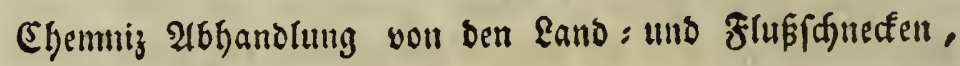
Tab. 127. Fig. I127.

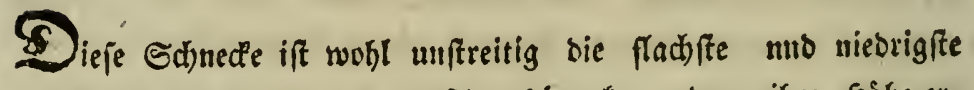

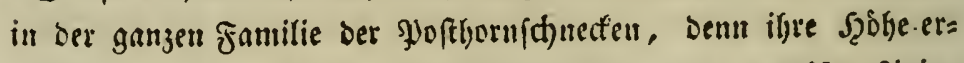

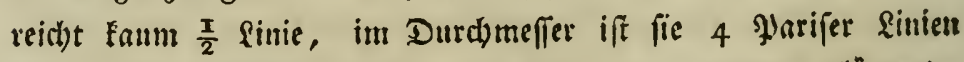
vireit. Die edjale ift ferg fein und zart, etwas glånzent, getGlidbrann, tellerformig, burdfideinerto unt feit in ber Duere geffreift. Die obere Seite if merflid anbserbobst, unten ift fie beynabe ganz flad), mit einer Sinnte in geraber Rinie ungeben, bie

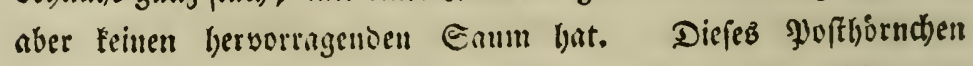

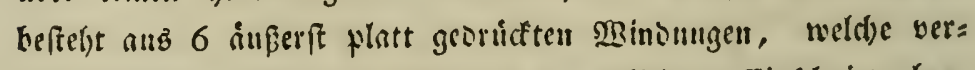

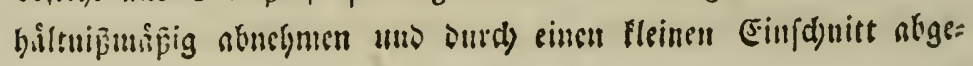




\section{$=35=$}

theilt find. Die obale geortidte Runboffnung geht wegen bet Sante etwas fdreg und ectig zu, uno ber obere Mhinounge: rano gelst ưfer iem untern wenig berwor. Dnz Thier ift roth

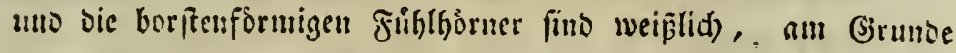

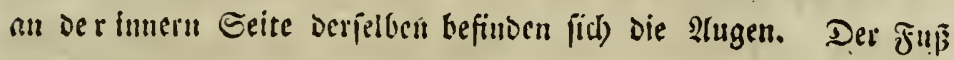
iff Furj uno geldt abgeftumpft aub.

Der Finbort bicfer Ylofthornden fino ftelenbez SBafler, Briben, Erimpfe uno Ieidbe, alt weld)en Stellen man fie nidys felten antreffen wirb.

\section{Helix lenticularis. Mihi. Tab. 2, Fig. 4. Die linfenartige Helix.}

$\mathfrak{A}$

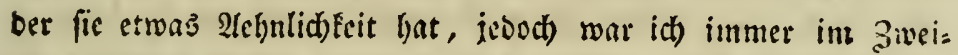
fel, weil bie in ber Bejdreibung angegebenen Merfmale bey ibr

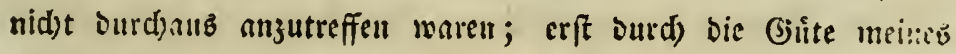

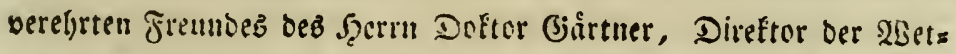
teranija)en (3ejellichaft für bie gejammte Raturkunde, in Şannu, Der fid) feit einiger 3eit nups serbienflic)fte mit Der unterfud)unj

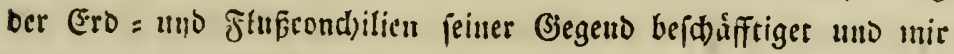

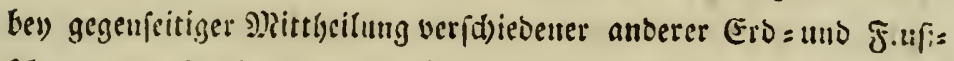
fdnecten aucl) bie wahre Helix nitida (weld)e in biefiger (jie: getto zur 3cit uod) nid)t gefunten wutbe) eingefantot hat - biut fd) nun z" bor sollen heberjengung gefommen, baßs sie inciuist nod) gat nidbt hejdrieten, fonsern vielmegr eine neue Epecico 


\section{$=36=$}

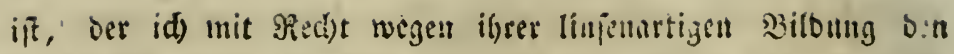
गtamen Helix lenticularis beylegge.

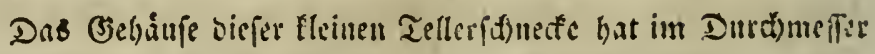

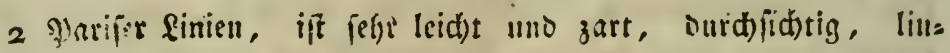
fenformig, glíujento, glatt, leid)t jerbred)lid) uno auf ber Dhet: fläd)e mit felle feinen Dueritreifutu serfésen.

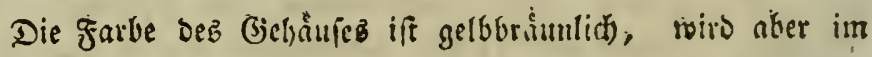

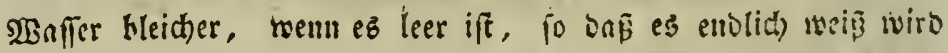
uno perlenmutterarting gliunt. Shit bem İbier but bie Èdyale eine sunfelbramte Jarbe, im IBBafler fejeint bie shiffte ser gres fen Mistroung ganj lecr uno ourdbfidt)tig.

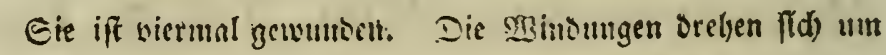

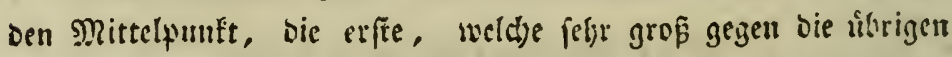

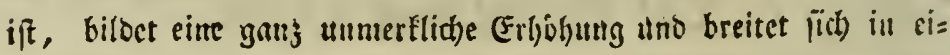

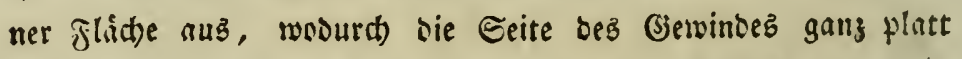
wirb, fie ift 2 mal gróper alz bie tibrigen, weldbe etmab tic= fer liegen. Die untere Esite ift ziemlid) flnd mo fo menig ers boht alz bie obere, woourch Die Sante, weld)e jugeipist ift,

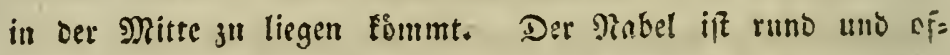

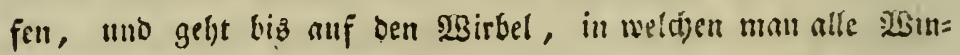
Dumgen bentlid) frhen. faum.

Die Runtoofnnung if fait bersfornig, fie hat feinen fip= penfaun, fondern oer Mlundungerano if vielmegr fdarf. Die obere Sippe, melthe einet ftumpfen 2 Bitfel bilbet, ift yon ser untern fel)r mertlich bervorgezogen.

Daz Thier hat in ber finge I Ylatifet finie unto eitue

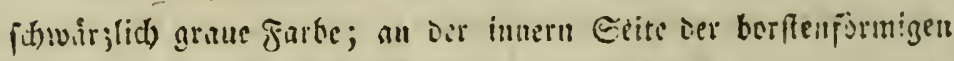

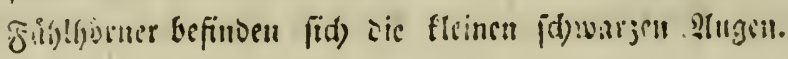




\section{$=37=$}

Şinter betr Sobel und bey Sangiveit, feitwártz bem 3oll, babe id Diepe Edurecte in fethenden Báffern, anf $\mathfrak{B a f f e r p f l a n =}$ zent unr felten angetroffen, lyingegen fand id fie sorziglid grof uno it zaflreider Menge in einem Sumpf bey Mithlbaupen an nltem bingeworfençn Graje, weldes burch pie fänge ber zeit ganz fdimarz geworben und einen moderiden (B) noumer batte. 


\section{$=38=$}

Helix Isognomostomos. Hermann. Tab. 3. Fig. 5 .

\section{Dit WinEellgarÉrn.}

H. testa fub fusca deprefsa: anfractu primo tereti, apertura cuarctata fub triargulari dri,tentata marginata.

Linn. Syst Nat. pag. 362 I. Sp. 158.

Klein Methodi Ostracolog. Tab. I. Fig. 22.

Diefe $\mathfrak{A b b i l b u n g ~ h a t ~ m i t ~ b i e f e r ~ S d j u e f f e ~ e i g e n t l i d ~ w e : ~}$ nig ঐehnliufleit.

Sdyroter Einleitung in ber Condhilien : Senntnif nad) Linné 2. Santo. pag. 194. 62.) Diefes Citat ift im Syftem nidjt angefulyrt worden.

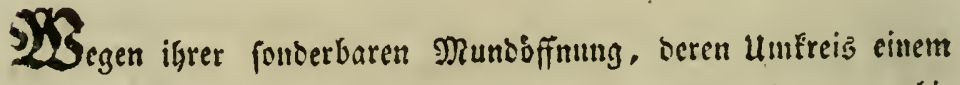

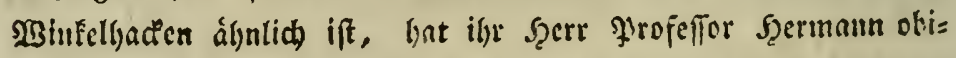

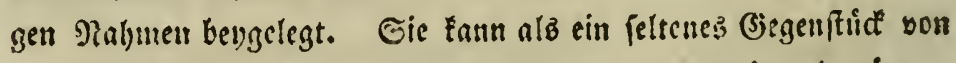
ser Helix obvoluta angefelgen wertell. Şgre Ejoßße betrigt 5 Parifer Sinien \$reite uno $2 \frac{x}{2}$ finie Şjbe. Die Edjale fat eime faft Erigelformige (jeftalt, ift son mittelmápiger Stáré, fel)e leid)t sefreift und bic Dberfladbe in ifyem ganzen Ulufange ift mit furjen, biditen, meiden Şäidhen bejetzt. Die lecre Sdjale ift etwab ourdjpheineno mo hat eine bramlide farke. Sie hat

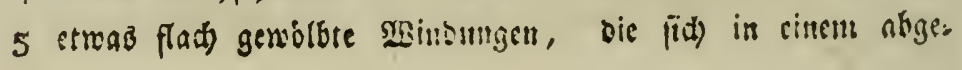




\section{$=39=$}

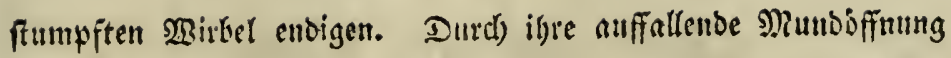
zeiduret fie fid ganz vorgliglid) aub, fie ift glcid)fam breyectig

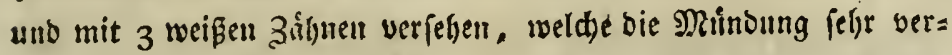
engern, 2 fleine ipitgige befincen fid) nuf beyben Eeiten be:

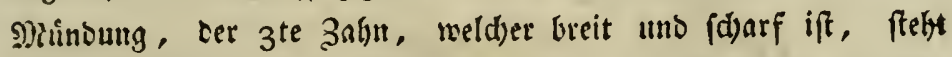
vorne querriber. Der Mlúnoungbínum, weldber einen fharfen

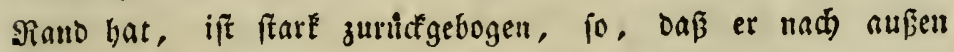

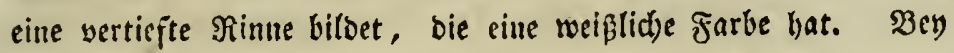
jungen unvollendeten Sdurecten fieht man jwar einet geoffneten Nabel, ber aber bey) vollig ausgemad) fenen won bem untgeid)(a= genen Mhinoungşanum Sfterb ganz bedectt wito, bey mand)en

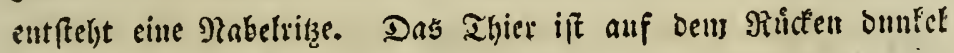

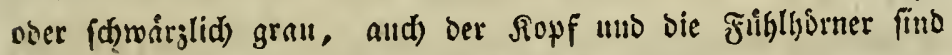
wou gleid)er farbe, bie Fubloble ift melge bellgran und geht in

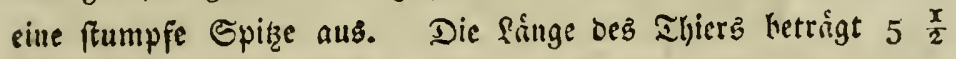
Jarifer Sinien, bie oberen Friblhorner mefin $1 \frac{3}{4}$ Sinie.

Stl unjerer Biegens ift fie bie grofite Geltentyeit; weninger felten fdeint fie jeboch in ber Gegend vou Stcinegg bel) \$fors= heim fu fenn, woher id mefrere Exemplare ourd) bie juscr=

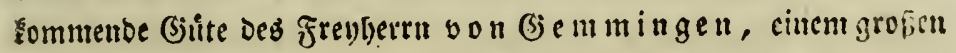
Sienner mo Sammler ber (Eondjilien, fo wie Berelyrer ber Bat=

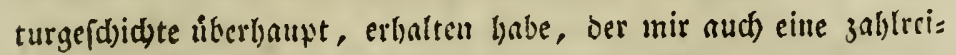
de Menge you Helix obvoluta henlegte. Son mir warbe iie,

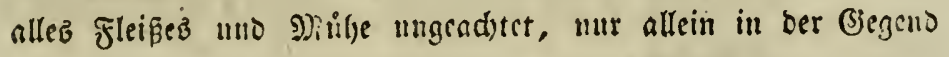
som Bugenberg in einem Thale an einem \$nd)enftumme suge= troffen. 


$$
=40=
$$

\section{Helix contorta Linn.}

\section{Dả̧ fleine biermal gemundene}

H. testa fubumbilicata plana utrinque aequali: apertura lineari arcuata.

Linn. Syst. Nat. pag. 3624, Sp. 37.

Flanorbis contortus Müll.

Das Eleine 6fad germunbene falfaje Pofthóndeen,

Müller Hift. Verm. pag. 162. Nro. 348.

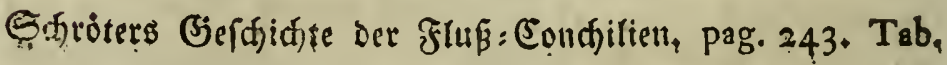
5, Fig. 29. utul pag. 237, XLVIII.

Petivere Opera Hist. Nat. Spect. Lond. 1767. Tab. 92, Fig. 8, Eine untriftige $\mathfrak{A} 6$ bilsung.

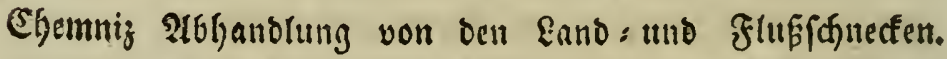
pag. 98. Tab. I 2\%. Fig. I 126, Diefe Stunes

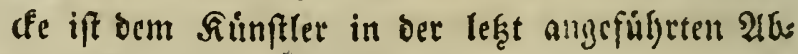
hanthung niche gut gelungen.

Bertinifaes Nagajin, 4 Sant pag. 259. Tab. 8. Fig. 21. hiet ift fie unridstig gegeiá)nt und enuflos geffipden.

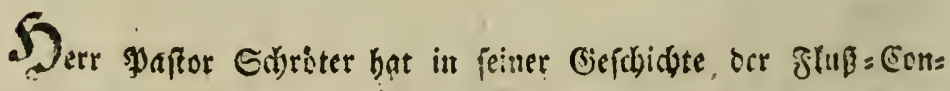
d)itien dic 2 obengenannten Bartungen getreint, on Iodh in Biruns

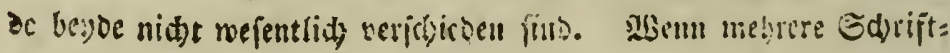




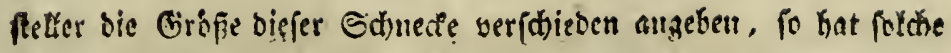

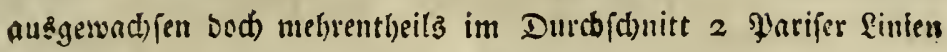
uno in ber Şoble beynahe $\mathrm{I}$ finie. Sie ift von ofen und unten platt,

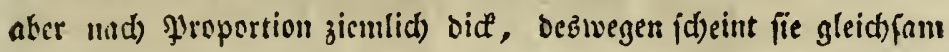

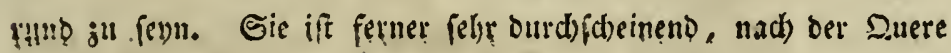

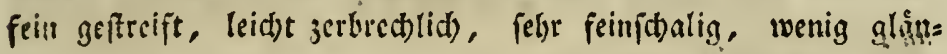
zetro uno yon hornbrauner Farke. Die Deffnumg mad)t mit ber Ş/erpentifulărlinie einen Winfel uno liuft gegen Die Dicfe ber

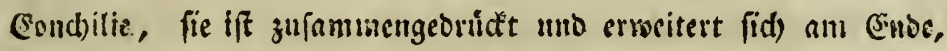
uno gleid)et, wie Linnć angenterft hat, einen Eateiaifden C. Plad)

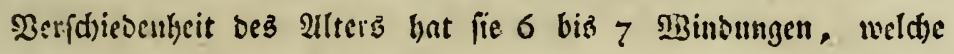

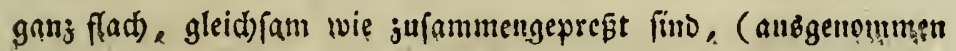

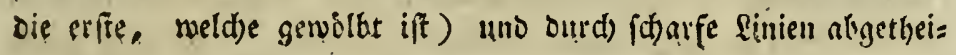

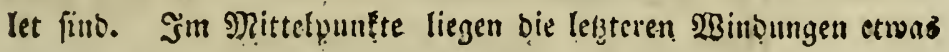
tisfer uno verurfad)en ein ganz lleineả. (jribibgen. Der untere

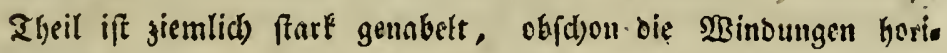
zoutal laufen; in bent Rabel, weld)er weit und tief ift, Fann

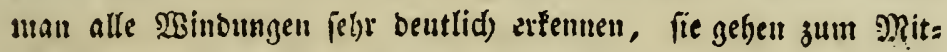
telpumft wie feine Enfin einet Treppe binnuter.

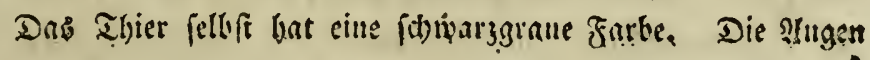

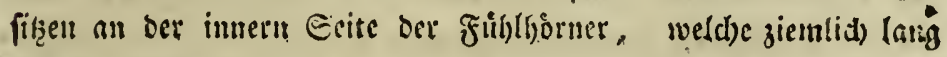

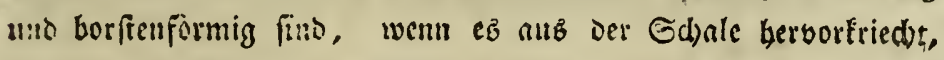

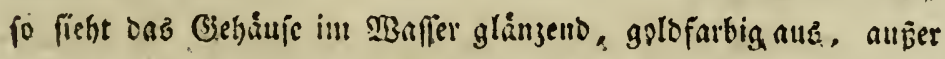
Dent $\mathfrak{X}$ affer hat bie Edale mit bem Thier cine braungelte Farke.

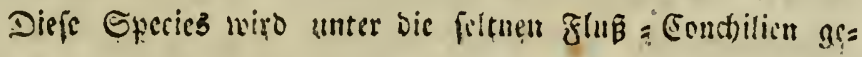
gered)net, in unfrer biegento ift fic an manden Porten jient: id bălifig anjutreffell. SMar fintot fie in Griben biuter ben fiobel

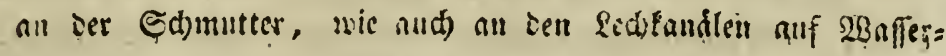

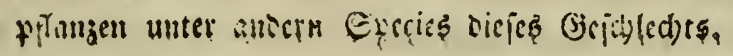




\section{$=42=$ \\ Helix alba. Linn.}

\section{Die weipe Telleridteref.}

tura dilatata.

H. testa alba, utrinque umbilicata: aper-

Linn. Syst. Nat. pag. 3625. Sp. $39^{\circ}$

Petiveri Opera Histor. Nat. Spect. London 1767. Tab. 92. Fig. 7. Dicfe abbildung ift undeutlid) uno feblerbaft, wemn es witflid) diefe fern foll?

Berlinifdes গagajin 4ter $\mathfrak{B a n t}$, pag. 253. Tab. 8. Fig. 23. cine undeutliche 2 dbbiloung. Durfte and vielleidjt cine andere Species fepn.

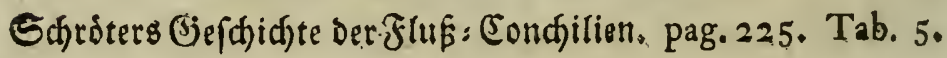
Fig. 12*

$\mathfrak{P}_{1}$

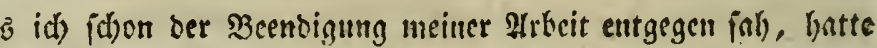

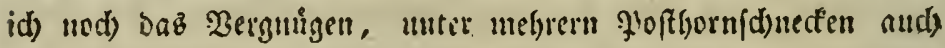

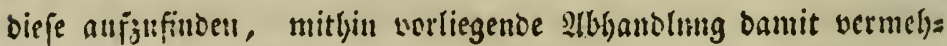
ren ju fonten.

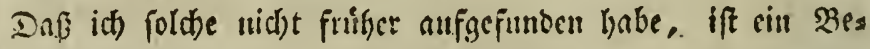
weiz, wie fparjam uno felten oicje Edonete in unfret Bicgents

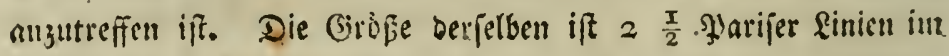
Durd)meffer.

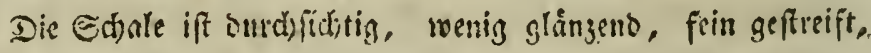

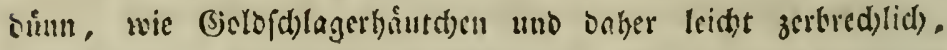
bornfarbig, oben und unten gleitaplatt ober poftlornformig uno 


\section{$=4 \hat{3}=$}

gegen sen Shittelpunlt auf benben Eeiten gleidjam etwaz cinges

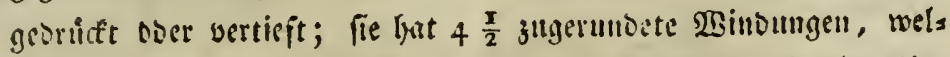

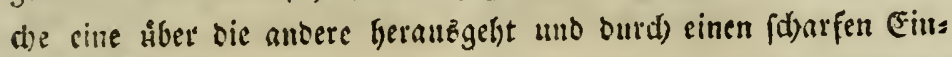

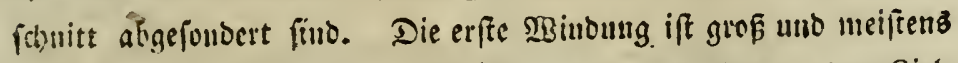
runo, aler bod) bišveilen mit einer frumpfen Sante vocr Siels runbe verféfen. Die ungefáunte Mundoffnung ift runolid) uno bie fdarfe Mintoungslippe fteht gegen bie rechte Seite weiter herbor, jcigt fidh folglid) ganz fdylef. Sie fojmmt in unfrer (jes getro fowohl als leere Edale als aud mit bem Thier ofterb gailj id)muthig, wie in Shlamme ungewoilyt, yor.

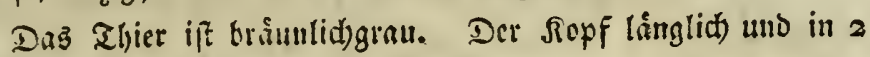
Furje runbe Sappen getheilt. Die Runboffnung befindet fid) in Der פnitte, unter oer Berticfung, woddhe bie bevbe Lappen yon cinander abtgeilet.

Sie bat 2 borifenformige weipliche frihlbormer, an beren immern Seite alt (j)runbe bie idiwarzen 2lugen ifren Silz baben.

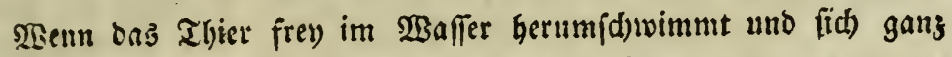

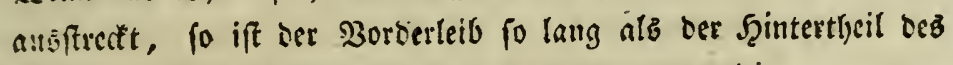

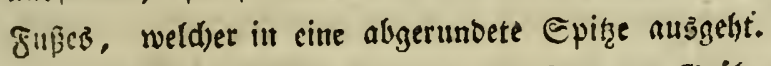

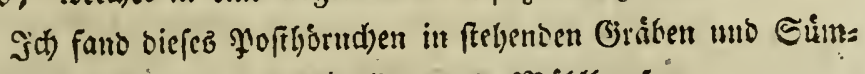
pion in ser Bregent non Eitegliag utro Mniblgaujer. 


\section{$\approx 44=$ \\ Helix hispida Linn. Tab. 3. Fig. 6.}

\section{Die ranke Ethntere.}

H. testa umbilicata convexa hispida diaphana: anfractibus quinis, apertuta fubbrotundolunata.

Linn. Syst. Nat. pag. 3625. Sp. 42.

Petiveri Opera Hist. Natural. Speçt. Vol. 1. London 1767. Tab. 93. Fig. 13. C. 105. fie ift ofne Scaace abgebildet utio ljat jut farke Suerftreifen.

Ginnani Opere Poltume pag. 59. Tab. 3. Fig. Nro. 21. Die Docrflád)e diefer Sduncfe hat ben biejem Situper in dem 2 serfe des Ginnani jul weit abfite: henbe Şaare, diel alfo wie Stacheln ausfeh)en, ałt ber erftert swindung babe id nidjt mebr wie adte gezahlt, audif) ift fie etwas zu grop vorgeftellt,

Schlotterbeck Acta Helvetica Vol. V. Tab. 3. Fig. 13. ift

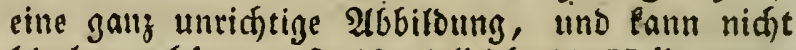
bierher geboren, fie ift vielleidgt die Helix turturum wẹil in Syftem bey diefer Sanuefe das nảm: liche Vol. Tab. unt Figura angegcigt ift.

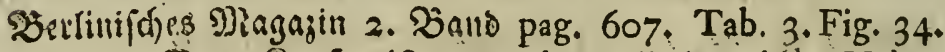
Das Supfer ift oem obigen in ber Acta Helvetica gants gleidg.

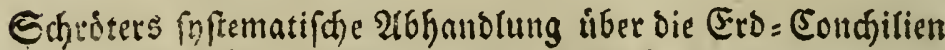
ttin Thangelftodt.pag. 186. Tab. 2. Fig. 21.

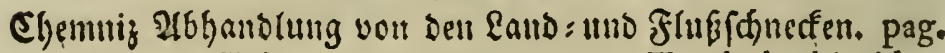
52.Tab. 122. Fig. 1057.1058. Trochulus hispidus.

$\mathfrak{B}$ matijd)en abganblung viber sic Erocondilien pag. 187. folgenbeb: 


\section{$\equiv 45 \equiv$}

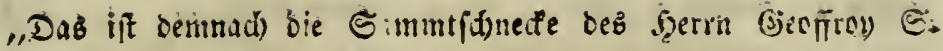

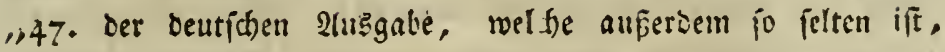
„Daß sifter, Slein, Seffer, Shartini uno amiere tum sic Cons

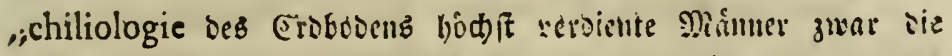
"glatte, aber nid)t sie hanrige gef̂̉mben uns bejhrieben haten."

Unt stugsbirrg ift fie nid)t feltèn uns auf maniben Ete:= len wohl gar jiemlid) báufig anjutreffen. Die Briobe belinif

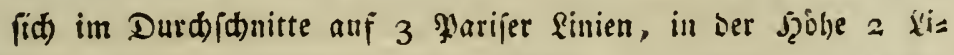
nien, fie ift rundtid), zart und leid)t, fein in ber Quere ge=

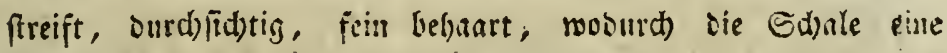

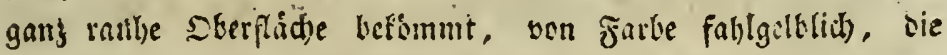

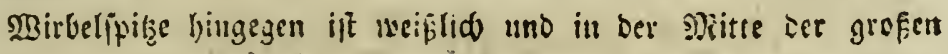

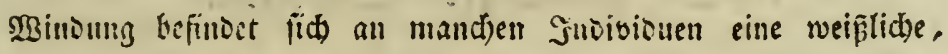
fdumale Binbe. Eie hat $5 \frac{\mathrm{T}}{2}$ etmas gewólbte 2Sinoungen, tie

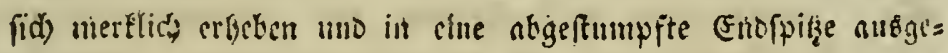

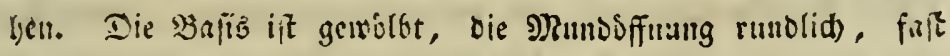

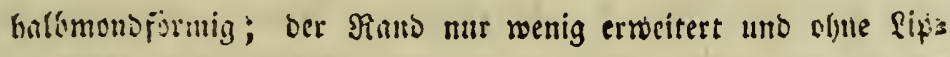
penjaum. Dr: Makel if juwar nut flèin, aber soch fo tief uns

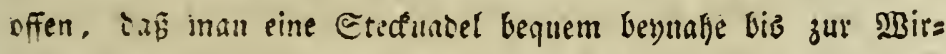

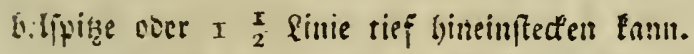

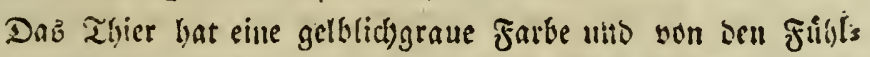

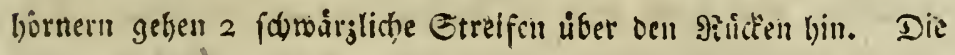

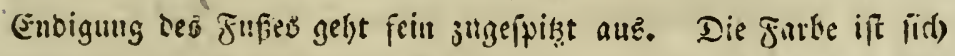
nid)t iumer gleid), Sfters jinbet nun aud) Edbuecfen, wo sas Thier oben fowars uniten aler eiue etwas belleve Farbe hat, soit

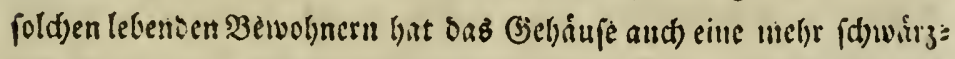

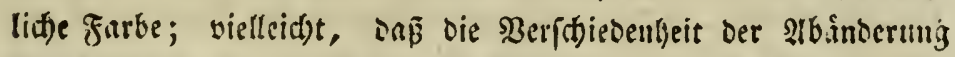

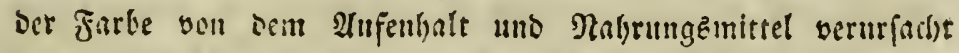

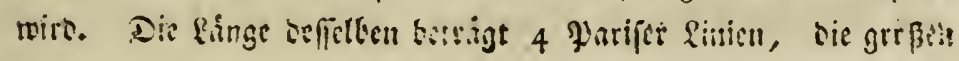




\section{$=46=$}

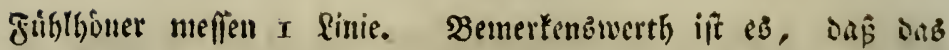
Ihier fegr empfindlid) und (d)idutern ift, weil es fich bey ber

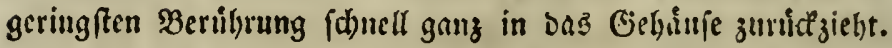

Man finbet diefe artige Sd)mede auf \$flanzen uno unter Bieftrånd)en, in Borbólzern Der $23 a$ rsungen, wie aud) an Ujern

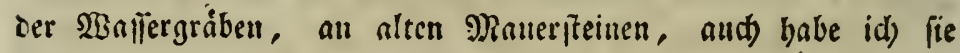
bisweilen in B̧årten angetroffen.

\section{Helix pilosa. Mihi. Tab. 4. Fig. 7 . Die banrigte Sannefe.}

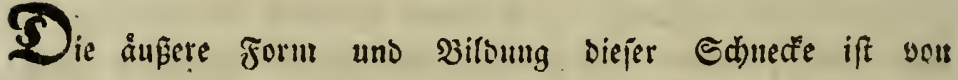
Helix hispida aufialleno uno mefentlid) veridbieben, oaljer ges wißß ber aufmerffumc Paturforfde: fein Bobenten tragen wiro, fie als eine eigne Epecies aufaufibren, weld)es id) aud) that.

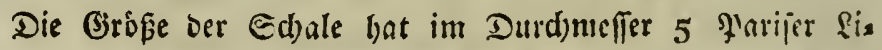

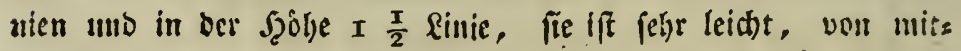
telmápiger Stárfe, ourdjid)eineno, in ber Ducre gefrecift, Hhas: Wrisunlid) von farte uno ilgr umfang ift mit gel(blid)en, glis:s zenten Şaaren bewachjen, berent Rinnge $\frac{x}{2}$ finic ketrigt. Sie

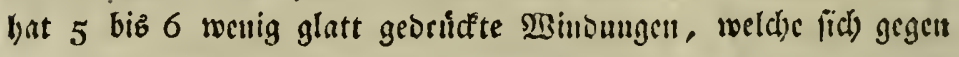
Den Mittelpunft proportionirt verminsern mo ourd) cincen fleinen (Einfd)uitt unterfd)iescu fins. Die şafiz ift ctwas gewilbt,

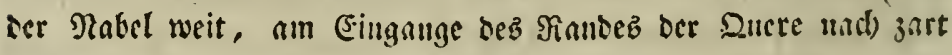
geftreift und reid)t bio auf sen STitbel herab, in weld)em man

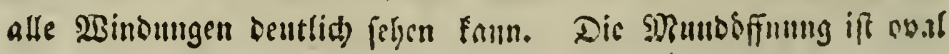




\section{$=47=$}

runslid, obue Sippeniaum, innerlyalb gelgt eine (d)male, mild):

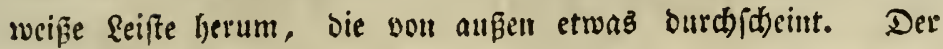
Mhinoungarano ift wenig elweitert ntwo an ber Nabelgegent ets

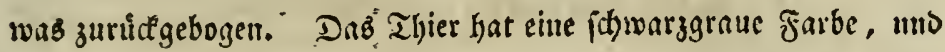
mit bemfelben ift bie Sd)ale fdwarrylid), won biejer Farbe aber

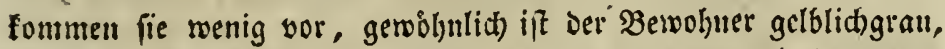

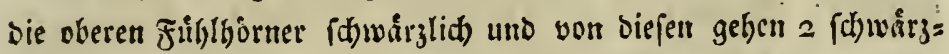
lidbe Streifen liber ben Riden lin. Mit biejen lebenden $\mathfrak{B} e=$

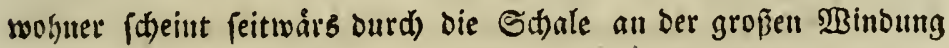
ein lánglid) gelblicher fled burd) und am

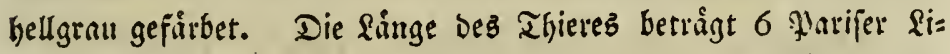
uien, bie gropen fillglgotme: meffen vollfommen 2 finten. Die Fupioble geht in eine ziemlid id)arfe Epize anb.

Man findet fie an fjattigen Drten in Maloungen, auf veridjiebenen Pflanjen, wie aud auf Erlen, befoubers auf SBei= Denftráud)en, an Den Rechkanilen auf bem Siebenbrunneufeld, wie and in ber Biegend you Stę̧ling. 


\section{$=48=$}

\section{Helix Pomatia. Linn.}

\section{Die Beinbergåfhnetfe.}

$\mathrm{H}$. testa fubumbilicata fubovata obtufa de: colore: apertura fubrotundo - lunata.

Linn. Syst. Nat. pag. 3627, Sp $4 \%$.

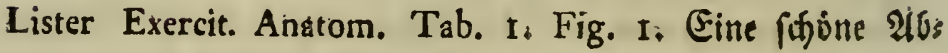
bitbung mit bem Ifjier.

Gualtieri Index Test. Cónch. Tab. I. Fig: A.

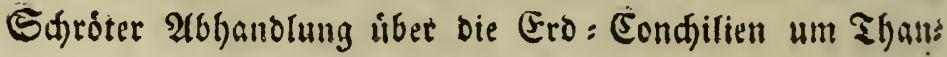
gelfeted. Tab. It Fig. IO,

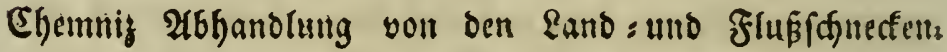
Seite 11 I, Tab. 128: Fig. I137, I138.

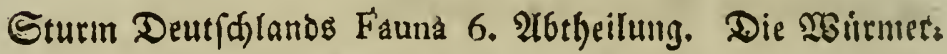
I. Şeft bas 13. und 14. Siupfet.

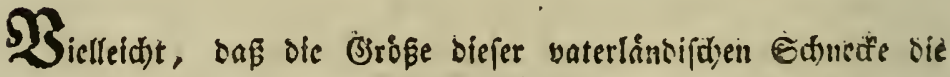

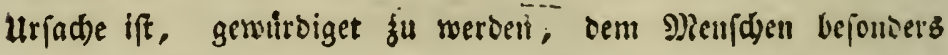
zur Fafenzeit zur Epeije zu bienen und zwoar nut bann, weent

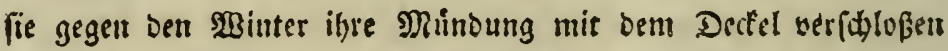

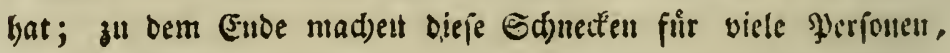

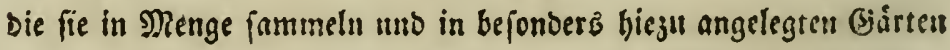
máften, bier, wie an omseren Drten, im Bropenen uno im Silets

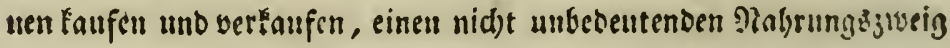

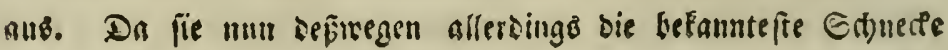




\section{$=49=$}

ift, fo enthalte id) mid), bieson mefrerb zu fagen uno fomme vielmebr gleid) an bie Bejchreibung ielbif.

Ins vorbanbene graste Exemplar in meiner Samminng

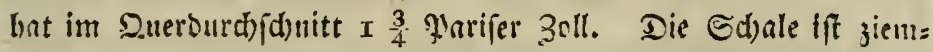
lid) ftart, menig glänzend, fugelformig, ithre 2lupenjeite ift mit bid)t anliegenten, lingsliden, grotent Etreifen ober Rumgeln

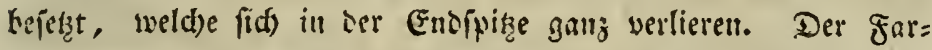

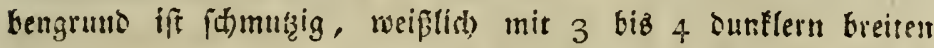

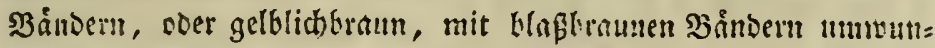
Deti. . Cie frat 4 biz 5 BBinoungen, movon bie erfie bauditg unt wie anfgeblajen ift, bie ubrigen fitto nur wenig serlangert

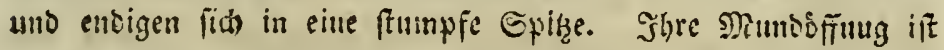
runolid), monbformig, furwendig weißlid) nutb glatt inz róth=

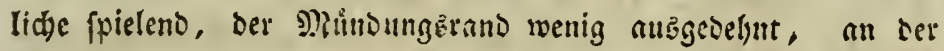
Epindel umgefdhlagen, woburd) eine tiefe, aber enge Natel= ipalte entftelyt. Daz Zhier lyat eine gel(blidgraue farbe und bie

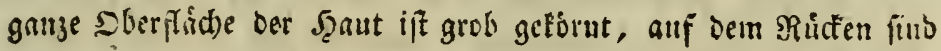

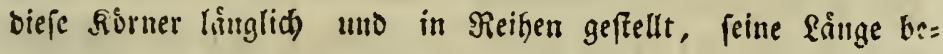

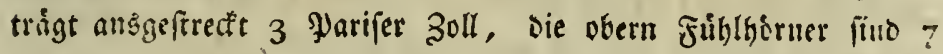

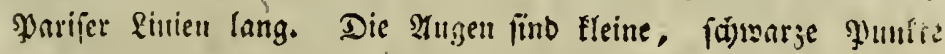

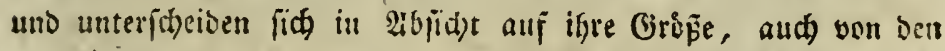

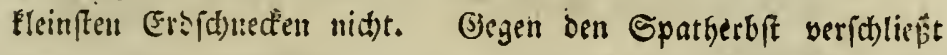
Dab Thier fein Gibrainfe mir einem weisen Fallartigen Dedel, wets

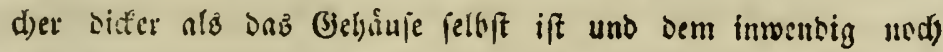

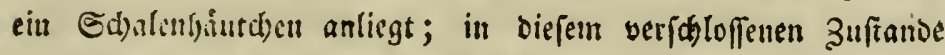

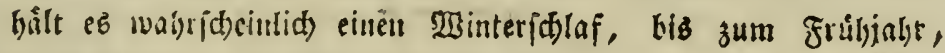
went aber warme ssitterung eintritt, fo lobt es Deut Dicfel felbje los, friner Nabrung wieder und)zugehen. 


\section{$=50=$}

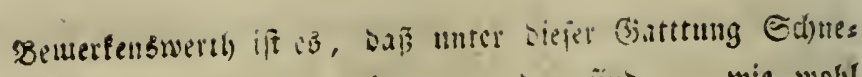

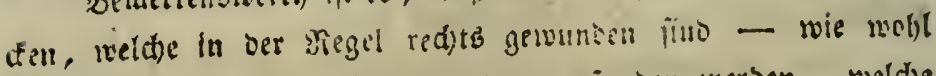

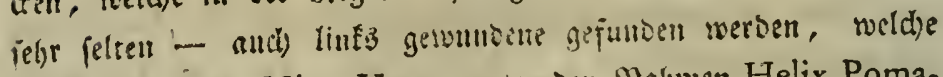
Mrifler in feituer Hist. Vcrm. unter orn Palmen Helix Poma.

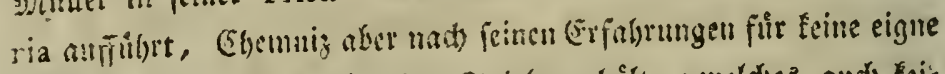
Epecies foubern blas fuir eine Epitlart hilt, ' weld)ez and) keis nem 3weifel unterliegt. Eis wito in unfret (jegeno zuar nid)t

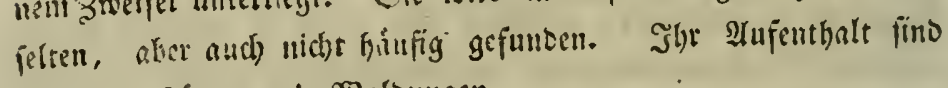

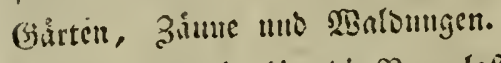

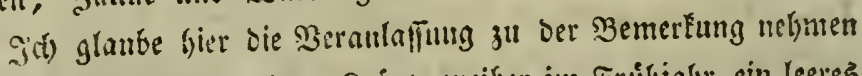

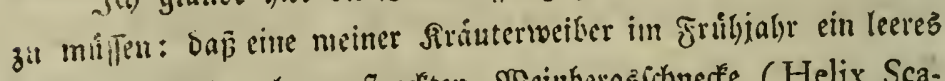

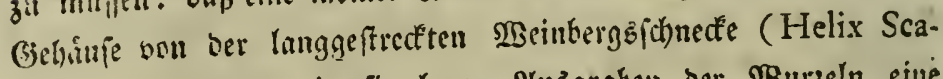
laris) gebracht, dic fie benm 2 (ügraben ber 28 urzeln eine

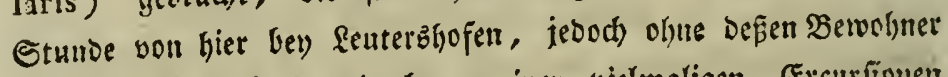
gefunden bat, warb mir bey meinen vielmaligen Excurfionen nie gelungen, felbe weber mit nodh ohne bas Thier aufjufinterr. Bey Ermanglung bez Bewohners unterlafe idh $\mathfrak{e b}$ mun, fie in biefer 2(b)andlung aufzunehmen, ungeathtet fid)'s vermuthen lápt,

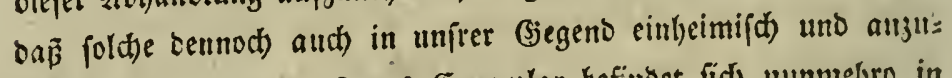

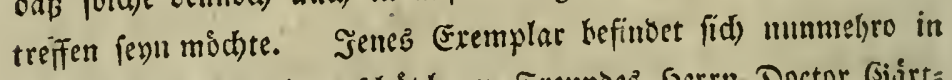

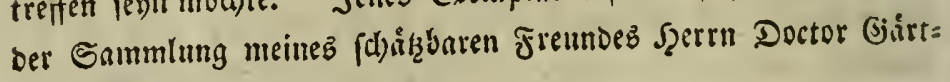
net in Şauna. 


$$
=5 I=
$$

\section{Helix Arbustorum. Linn.}

\section{Die geflette Sartenifinedfe.}

H. testa umbilicata convexa acuminata: apertura fuborbiculari' bimarginata; anterius elongata.

Linn. Syst. Nat. pag. 3630. Sp. 53.

Gualtieri Index Test. Conch. Tab. I. Fig. D.

Bevens monatlitie Beluptignngen. Tab. 30. Fig. 345 $-356$.

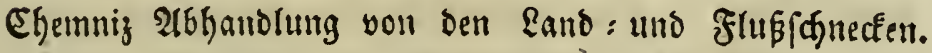
Seite 148. Tab. 133. Fig. I202.

Sturm Deutfdrlandos Fauna 6te 2 trtheifung: Die wairmer. I. Şeft. Das I 5 Siupfer.

23.

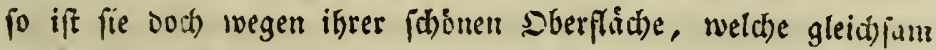
wie alf oas fleipigfte gemaglt erid)eint, eine unjerer ziertlicíften (Erofönecten.

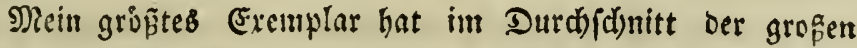

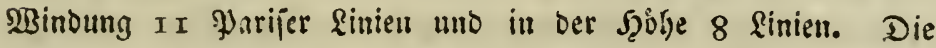

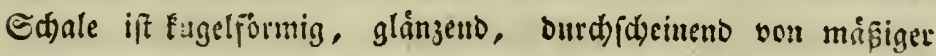
Etâre unto mit länglichen Etreifen serfelgen, welde an ber 9tatl) herum gleichjam wie eingeferbt fins.

Dic smonboffnung ift montiformig, bie sippe etwas unr

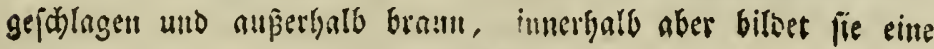




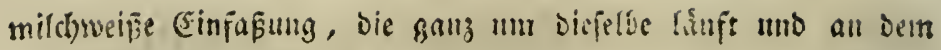

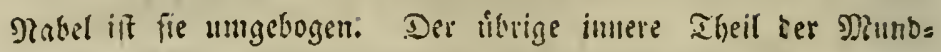

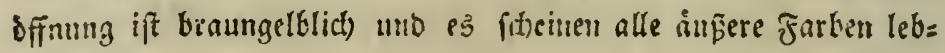
baft and) imwendig burd). Cie hat 5 bib 6 MBindungen, wo= son eine iffer ber anbern hersorragt, meidfe fich in eine ftumpfe Epize enoigen. IIn ber Seite oer Epinbel ifi fie nur wes nig genabelt, oocr hat eine vercugerte Şoblung, ben manthen

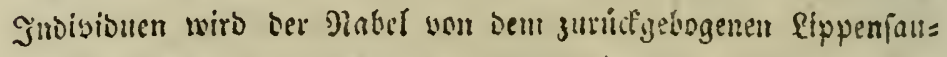

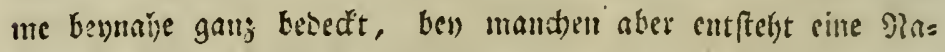
belrize. Die farben fint verinberlid), wenn fie aber villig

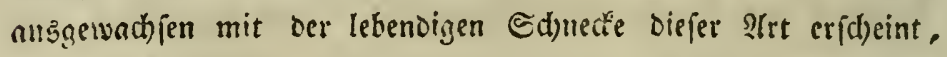
fo if oer Brund hell Eaftamienbraun, Durd querlanfente

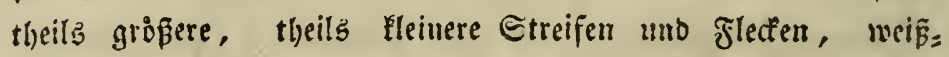
(id), gelblid) uno bráunlid) auf eine fely: lieblide sirt gleid) fam marmorirt. Sie bat eine eimzige gleid)måñige uno jhmale Binte, weld)e son ounfellurauner Farbe if uno von ber Mitte ber gropent BBinoung fpiralformig herumlåuft. Då Thier hat cinte eijen=

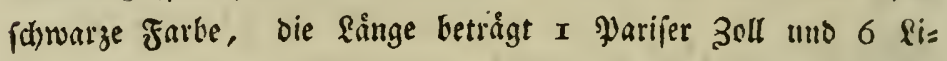
nien uno wiro son (Eibed) jen uno (Enten gefreffen, und zu ihrer

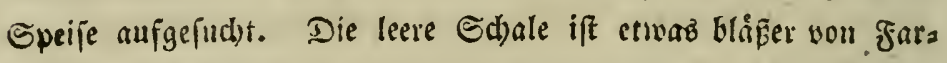
be alo biejenige mit dem Thier. Dod) bemerft man aud) an mandhen Exemplaren wenig llnterid)ieo.

9) ian trift fie laaufig in (joårten, an fdattidten, fend)=

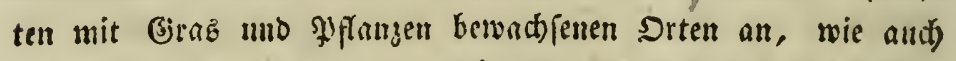

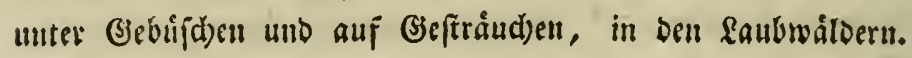




\section{$=53=$}

Helix nitidula. Mihi. Tab. 4. Fig. 8.

\section{Die giglittete Edstitifellidmece?.}

Sa id) in feinem ocr beutfdyen condjilienwerte weter cinc

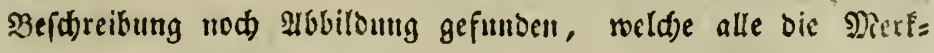
male biefer Sdnnecte, bie id) yor mir liabe, in fid sersinight bitte, fo glanbse id midh beredjtiget igh biefen fegr paffenten Nlament z"l geben.

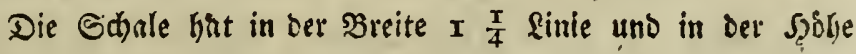
I Sinte. Sie ift ziemlid fungelformig, outchfidhtig, felje fein= fdhalig, fehr glánjeno uno glatt, Keynahe ganz olyne Etreifen.

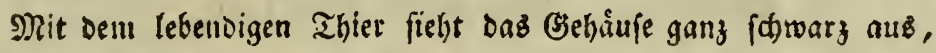
menn es aber bavon befreyt ift, fo hat bie Sd)ale eine rottly= lidgelbe Farbe. Sie bat 5 bis 6 didht cinander geidhlofiene fpis ralförmige, erhatene $\mathfrak{B g i n o u n g e n , ~ w e l d ) e ~ b u r d ) ~ c i n e ~ f e i n e ~} \mathbb{R} i=$ nie gettennt fïl uno in eine ftumpfe Spize anşgchen; bie Runosffunng ift enge, faft halbmonoformig, ber Rand berfelthen iche binne und oljne Rippeniaum. Dic Gruniflid)e ift cons vex; yom 9tabel fitbet fich nur cime Spur, aher cr ift ganz werwad)ien, ben mimlidjen fall benterft man aud bey jungen

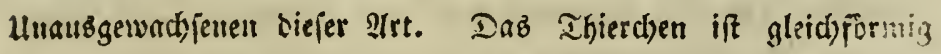

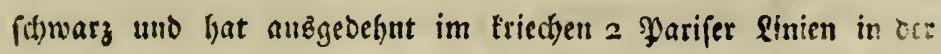

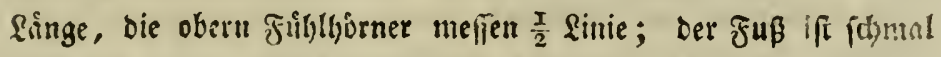
uno geht lang jugeipiģt aus.

Man fintet fie an feudten Drten unter Geblifhen auf sls ner lodetu, fanbigen Doorerbe mit abgefallenem faub besechs, ift aber in unfer Giegeno felten anzutreffen. 


\section{$=54=$}

\section{Helix Ericetorum. Lister.}

\section{Die Sheiderduncte?}

H. testa umbilicata depressa lutescente: fascia una vel pluribus fuscis.

Linn. Syst. Nat. pag. 3632 . Sp. 65.

Lister Hist. Anim. Angl. Tab. 2. Fig. I3. Die Munds

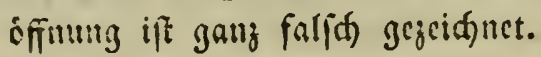

Gualtieri Index Test. Conch. Tab. 2. Fig. L.

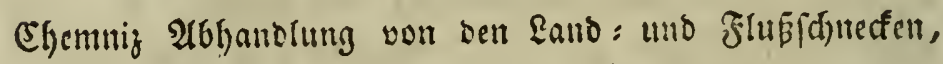
Seite 143. Tab. 132. Fig. I I93 - I 195 ,

Sturm Deutiálanos Fauna 6. Abtheilung. Die నrutr mer. 2. Jgeft, das ste Siupfer.

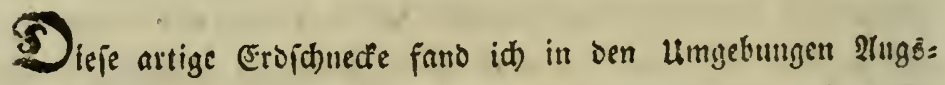

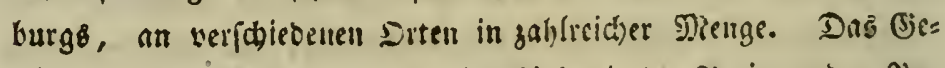

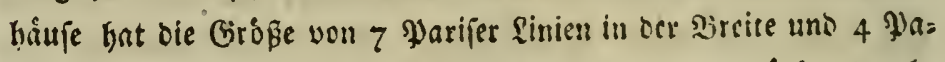
rifer linien in bet Şobe, fie ift von ziemficier Srîrle, Durd)= fdeeineno, etwas glaujent, glatt, pofthoriformig mb hat. Durd)aus fefre feine Duerftreifen nuf ber Dberflaitis. Die Numb= offnung ift oval rumblid), ber Rand zugefdjaifit olyne Enum, unb nad) oben jull vcrtángert, immensig aber mit einer glán=

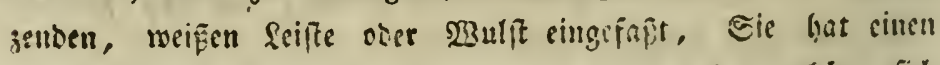

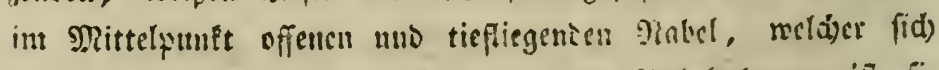

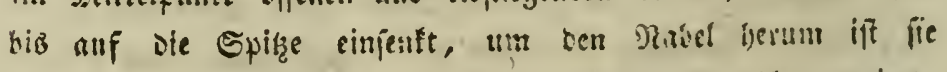
mit vieleu concentriften uno fein gringten Errullen grjiert,

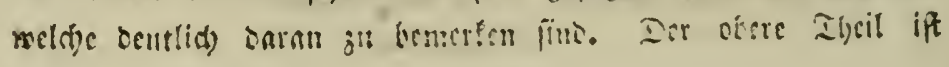




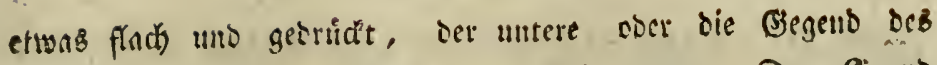
siakels ift gewollt. Sie hat 5 BBitroungen. Der Bimo

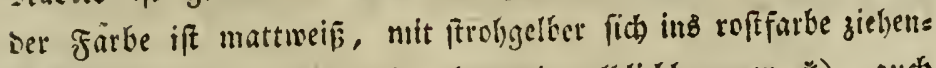
ber Edattirung, intggemein aher mit gerblid)braunen *) aud fdwarziramen Biftern gehuthen, aber bie Sronung uno bie Stujabl biefer brounen Sainter variren angerotbentlid), ob fie

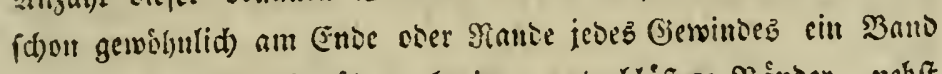

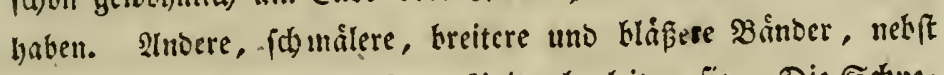
aแs \$umften zufanmengefetigre Sinien begleiten fie. Die Ed) de jelbft hat eine gethlid)graue Furbe mol ift 7 Syarifer Sinien

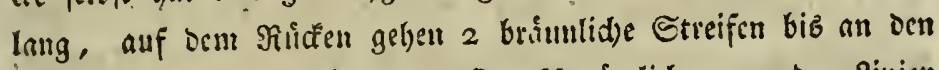
Mantel, sie oberen Fihllhornei fino idjwárglidgrau uno 2 linicn lang, die unteren finto etwab heller son farbe. Der Şinter=

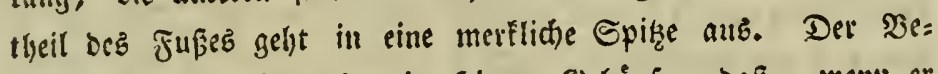

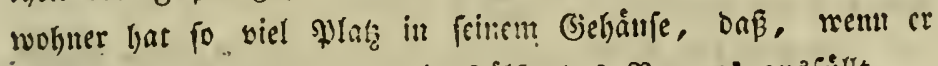

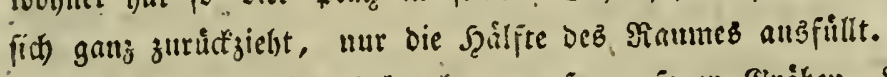

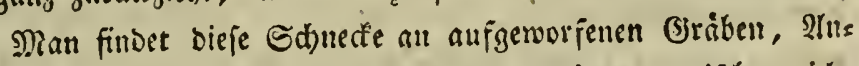

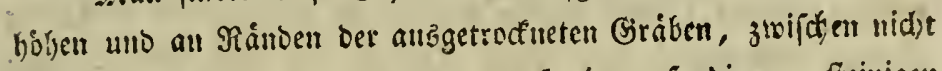

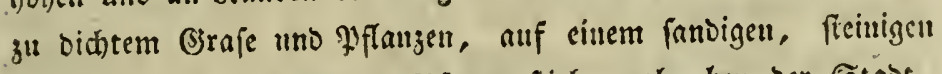
Croreld, am fogenanuten Wfamenfiticl, wahe ben ber Stabt, wie aud) bey bem Sickenbrumenbao und in ber Gegend yon Ereţling habe id) fie am båufigften angetrofienet.

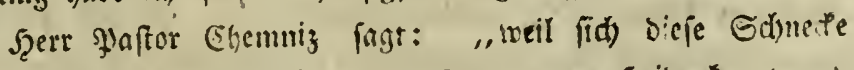
„, am liebften bers ber Erica oocr ingenaunten Szeidenfraut uno

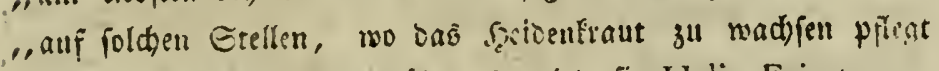
"(in locis ericetis) anfgilt, fo wiro fie Helix Ericetorum

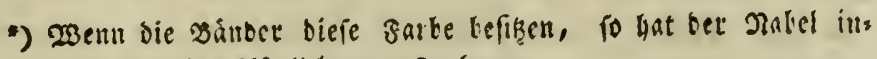
melloig cine blikulitgraue zalle. 


\section{$=56=$}

"genunnt:" wildes ater son mir nie beobaditet wurbe, obs gleid) melyrere Sgcibentten in Menge um Ilugäburg radjén.

\section{Helix Thymorum. Mihi. Tab.5. Fig. 9.

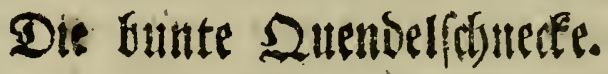

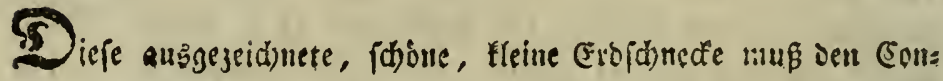

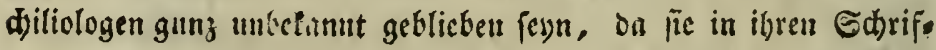

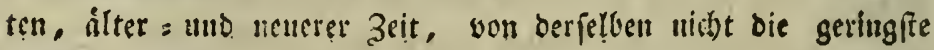

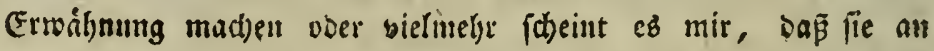
wenig Drten anjutreffen, mitljin feljr felten ift.

Beil fid birfe Eduredte am liebiten am Thymus Serpillum aufallyalten pifegt, fo habe id) fie bepiwegen Helix Thy. morum geuant, Jibre Giriße betrigt in Durdymefier 3 Yarifer Rinien, uno von unten, wetn mall bon ber skinoung biz zur

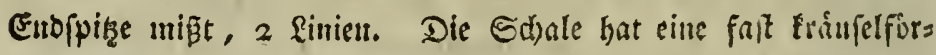
mige Geftalt, ift von ziemlicher Etärfe, etrogs surd)intsentento, wenig glänjeno, mit didten bejetgten Suerftreifen verfeben

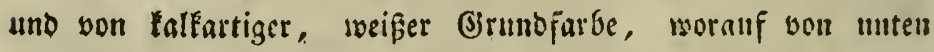

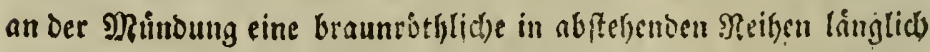

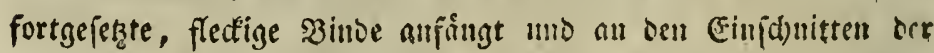

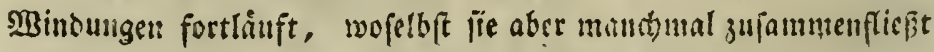
uno biz jut Enbjpike, bie kraumsolh gefistot ift, fonctls

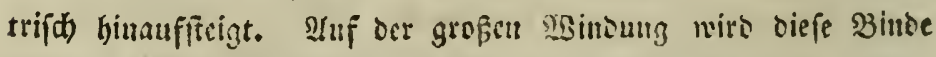
in zwey Zheile getrennt, oser glcit) fam wie wry eiuter weipen

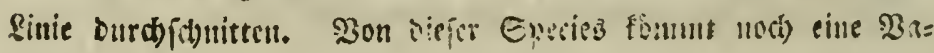


rietit sor. Sie hat ehenjäls eine meife Farbe, wie gebrann=

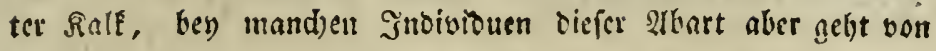

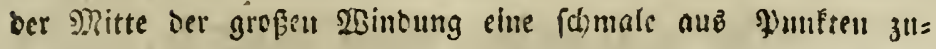
fammengeperzte sinis, meld)e fpiralforming um bie MBinoun= gen herumlanft uns fid) gegen sic Endpighe zu verliert, wel= d) ench) ofters braumroth gefirlit ift. Sie bat 5 flad getwolbte

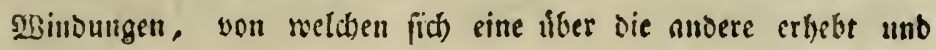
entrid) in eine abgeftumpfte Spikze nuingebet. Sn ber Mitte Der 3 afiz, weld)e gewollet ift, befintet fid) cin fleiner, enger uno ticfer গlabel, in weldsent man die Beswinde nicht bener:

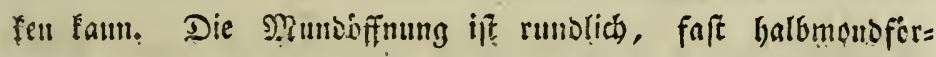
mig, an ber innern Seite berjelthen ift fie von einer weisén feifre eingefabt, fie hat feinen Mindungbianm, fontern bes

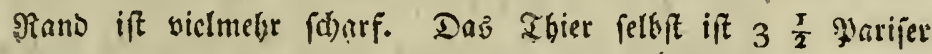
Linien lang, bic oferen füblhơrner mefien sine Sinie und eş ift

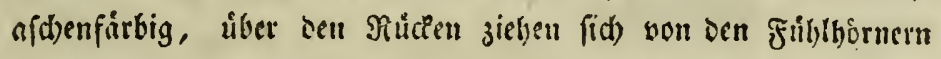
2 id)wirglidgraue Streifen, an biejem Drt ift bie Farbe etwaz bunfler und mit einer helfern Farbe wie melitt oder gleidjam

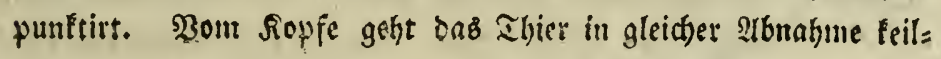

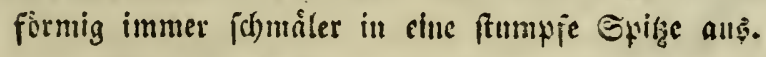

Relyrentlyeila finset man fie an Duenbel (Thymus Ser. pillum) wie anf anbere! nicbrigen P̧lanzen an gebirgigen Bies genben unb nuf 2tubshen, befonders batie id fie an Rofenan= berg bey 2tugghurg, wie and, abcr feltrucr, auf Ebenen in

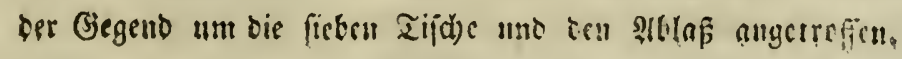




\section{Helix nitens Argenville. Tab. 5. Fig. 10.}

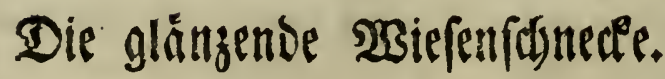

- H. testa umbilicata fubdepressa fulvo cornea pellucida fubftriata: apersura larga. Linn. Syst. Nat. pag. 36.33. Sp. 66.

Petiveri Opera Hist. Nat. Spect. Tab. 93. Fig. 14. C. 104.

Sdwammerban jeidnete biefe Sdnncfe in feiner ßibel Der Natur. Tab. 8. Fig. 3. Diefes Siupfer giebt aber nach meiner Einfidft z̆u irrigen $\mathfrak{B}_{0}$ :

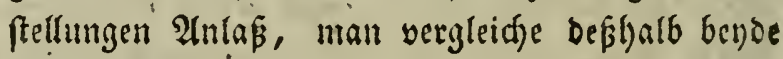
miteinander.

Argenville Conch. Tab. 28. Fig. 4.

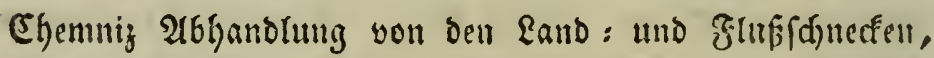
pag. 103. Tab. 127. Fig. II $30-$ II3I. Helix nitida.

T)

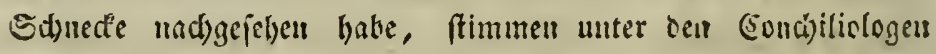

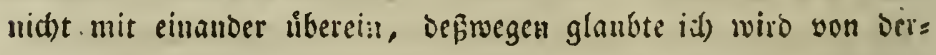

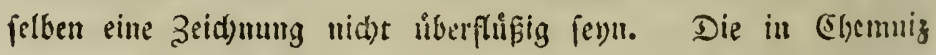
2thbandung vorfomikende zrid)nung, Das bie bogenformige Rumboñnung von einemi blapprothlid)en Sanme eingejapt wirb,

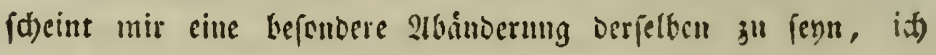




\section{$=59=$}

fand yor inctrern Gahren einige leere Gerháufe bie mit biefer

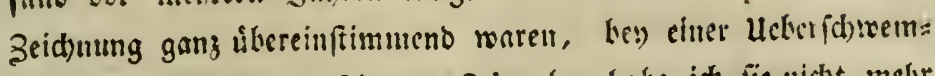

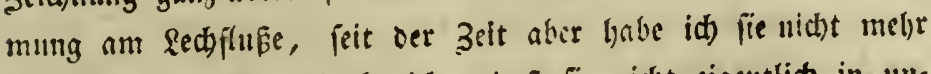

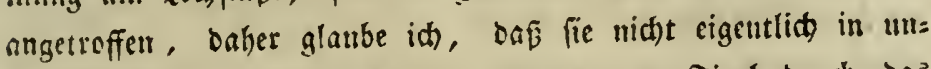

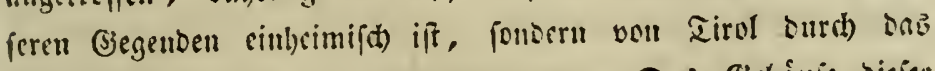

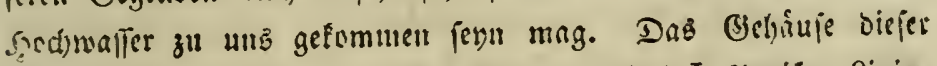

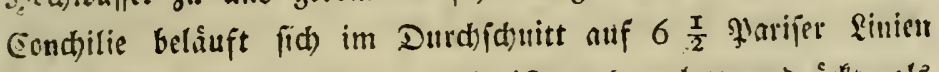

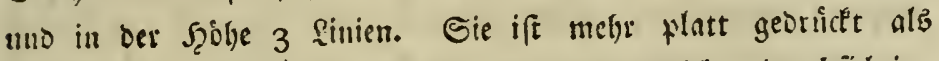

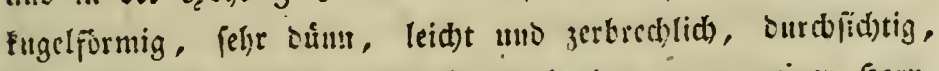

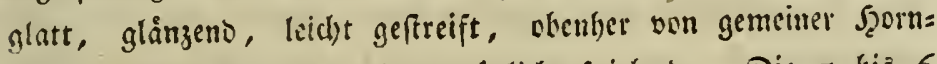

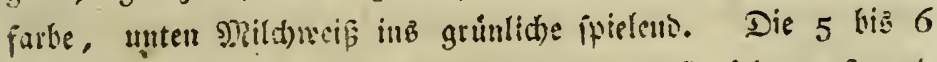

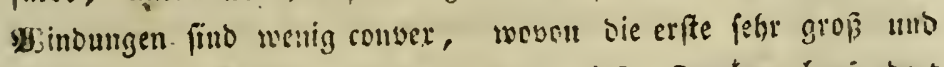
aufgeblafen uno surd) eine etruas tiefe furdte ahgejortest

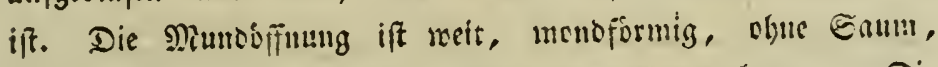
ocr obere Seitenrand geht sem unterm etwna hervur. Die Grunbflade ift menig gewoltbt. Der giabel liegt anfer sem

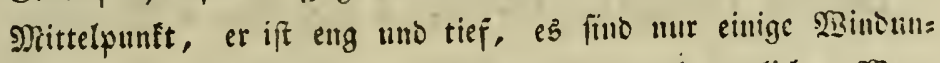

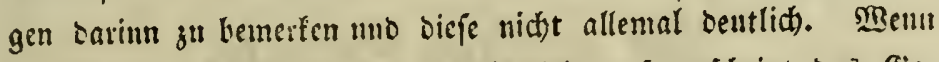

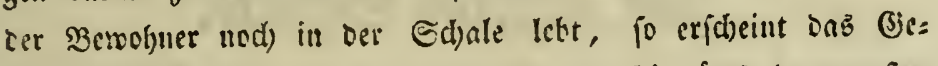

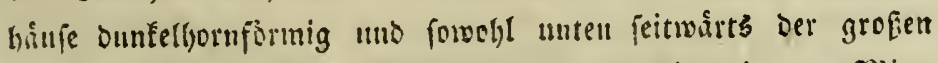

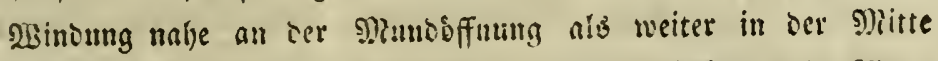

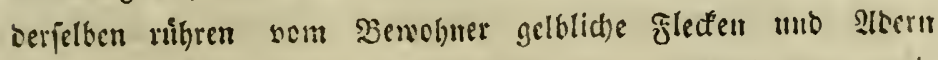

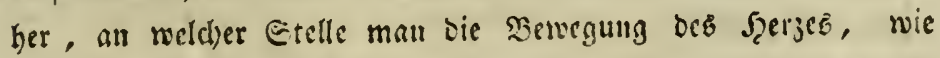

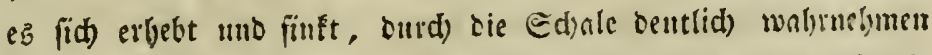

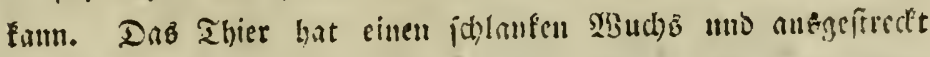

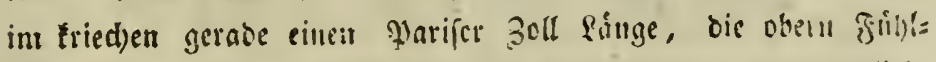

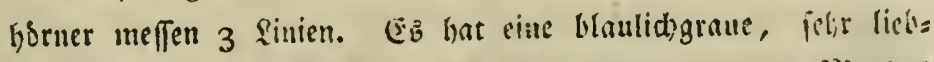

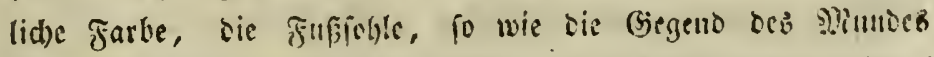

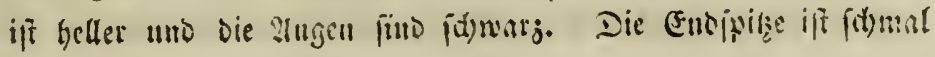




\section{$=60=$}

gulaufend und io lang, doß fie 3 Parijer finien hinteu unter bem Bẻjáuje binauछrridjt.

Unter feud)ten alten Mauerfeinen, faulem Şolj, fo wie an ben 2 Burzeln ber Brenueffel findet fie fid, aber nie im sizaffer jelbft, fondern immer auf ber Erie, hejontere an ei=

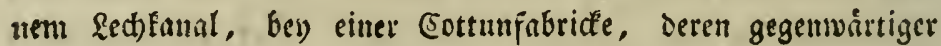

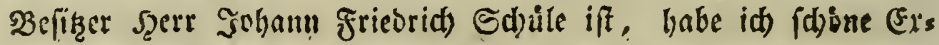
emplare angetroffen, aud) ift fie it unjer (segento nidht felten auf manden anderen Etellen. Merfwuirbig ift ifje außerorbents lidbe Bermefrung, weld)e id) in cirem Salten, ber zul meinen Beobadtungen ủber biefelben gehalten miro, malynahm. In biefem Salje wenigfenen entftanden aub einigen glaaret eiue jalliteide Menge.

\section{Helix costata. Müll. Tab. 6. Fig. Ir.}

\section{Die gerippte Sodnetfe.}

H. testa fubdepressa umbilicata cinera anfractibus costatis, apertura circulari: labio albo reflexo.

Linn. Syst. Nat. pag. 3633. Sp. 67.

Müller Hist. Verm. 2. pag. 31. Nro. 233.

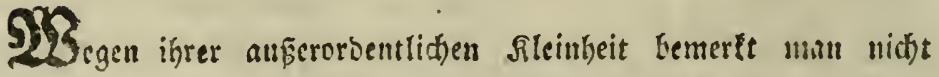

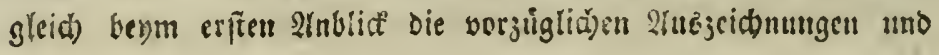




\section{$=6 \mathrm{t}=$}

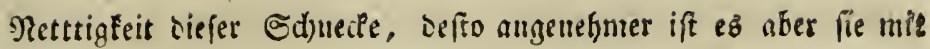
bewafuetem Inge 3n fierrad)ten uno liefonders mit einem 3 rans

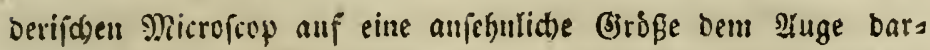

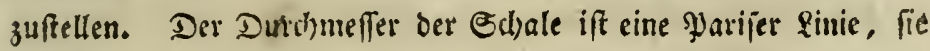

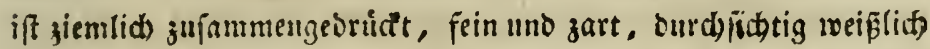
ober hellajabengrau, wenu aber dab bebáaje nod) von sent le: benven Thier hewolnt ift, fo lyaben bie leghtere $2 B$ intoungen eine gelbliche farke. Der llmfang hat beutlid)e regelmåaige Duet: rippen, man fann an der erften 2 Binoung 30 bie 32 zábien, tvie diefé von mir ourch) oas Eonnenmicrofop mit siner eignen

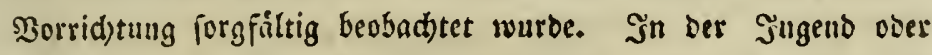
wenn fie nod) nid)t vóllig anb̧gewadjien, ift fie fein ge: fireift uno sic Rippen uidtt fo Deutlids walyrgunelsmen, alb an ben nusgewad)jenen Exemplaren. Dod) finder man fie auds

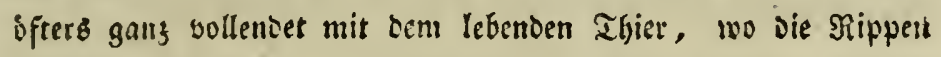

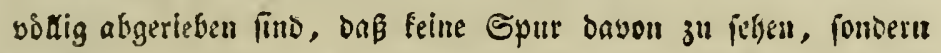
vielmeht die Dberfládje gans glatt, glanzent uno wie politt er: fdjeint, uno bie Mniffer in feiner Hift. verm. mit unred)t als cis ne eigne 2 rt (Helix pulchella) aufgefullyrt hat, weil alle ubrige Nerfmale mit jeiner Beidreiklang jelbft úbereintreffen. Cie ift viermal gewunben, bas erfte (serwinbe ift fer)s weit, bie úbrigen erbeben fid wenig uno endigen fid in eitre ftumpfe Endpikize.

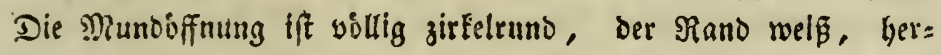
vorftehent uno zurrifgebogen. Der Ptabel ift ganz offen uns

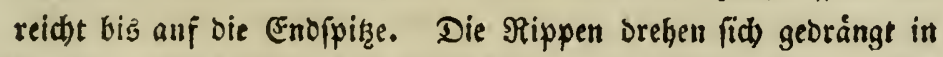
Den Mabel binein uno verlieren fid gegen die Mitte zu. Das Thierchen bat eine \&ange von $\frac{3}{4}$ Parifer Sinien Ift Durd)aub meiß

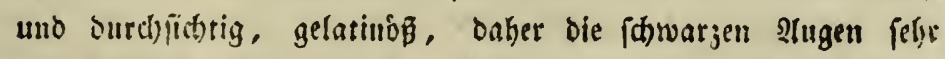

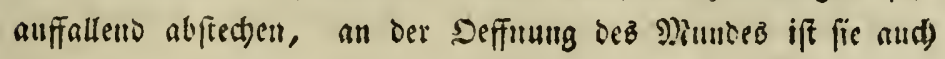




\section{$=62=$}

menig gefairtr. Sie bat vier friblhorner, wovon bie unteren

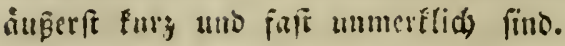

Cie if in unirer resegend elen nid)t felten, man findet fie ain Ulfer ber Edmutter, ber $2 B$ ertad) unto binter bem fo=

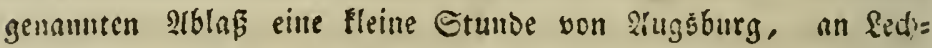
fanalen, auf (s)rab, Pllanjen uns bemoften Steiner.

\section{Helix rotundata. Argenville.}

\section{Dét Rnopf.}

H. testa umbilicata fubdepressa: lineis transversis elevatis maculisque ferrugineis.

Linn. Syst. Nat. pag. 3633. Sp. 69.

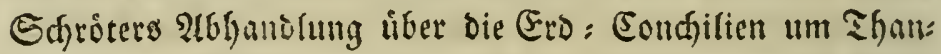
gelfted, pag. 199. Nro. 68. Fig. 25, Die fleine aflatild)e Schlange.

Argenville Conch. 2 Theil, Seite 67. Tab. 9. Fig. 10.

Sev einer gennuern lunterinchung habe id) mid) balb tiker=

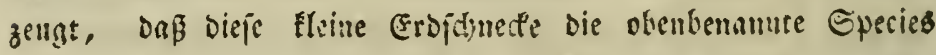

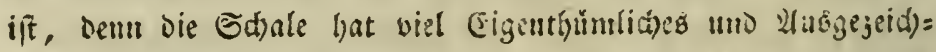

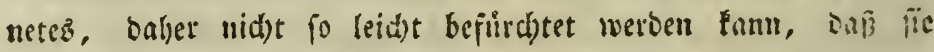

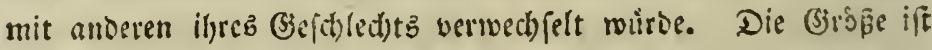

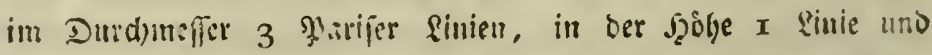
wito uid)t lcidt grofere: gejunter. Eic ift rmto, wenig plat, 


\section{$=63=$}

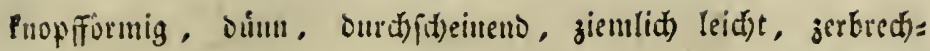
lid) nid)t glánjent, auggennumen bie Enoipizize. Die gauze Dber= flicbe ift mit bidt) bejeţten, hervorragenden Durrjteifen un=

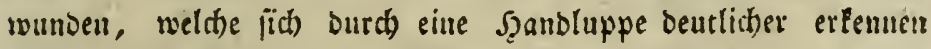

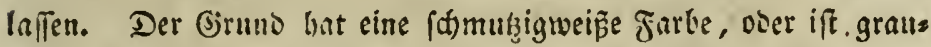
(id), auf wel(h)en bräunlid)e folectgen in gleider (Ent fermung ver= theilt fint, bie anf allen STbitbungen biz an-bie Enofpitge fortlanfen. Sie bat 6 wenig convere Binoungen, welde ith

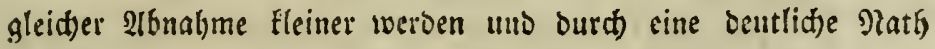
yon einander abgejonbert fint. Die (Brunoflict)e if menig con= ver. Der Nabel, welder weit uns trichterformig ift, geffet Durd) ifren ganjen Bau mitten binourd), in weldem nuan alle 2Binoungen Deutlid) Kemerfen Eann. Die Nunboffnung ift jiem= lid) gerutbet, wenig erweitert, fuft halbmontoformig uno wirb yon Eeinem Sippenfaum umgeben. Die obere Sippe geht ber un= tern etwas bervor. Die Ráuge deş Thierdhens ift $2 \frac{\mathrm{T}}{2}$ Marifer Einien, Die obere f̧üblgôner meffen $\frac{x}{2}$ Sinie. Der Sopf und

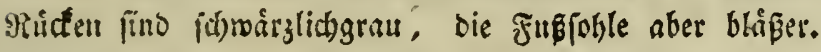

Shr 23ohmert find ujer ber Baffergråben, auf faulem

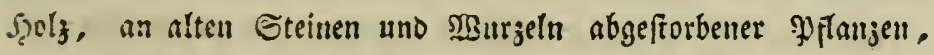

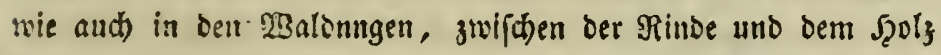
jauler abgehauener Baumftámme, an weld)en Drten fie nid)t pelten anjutreffen ift. 


\section{$=64=$.}

\section{Helix obvoluta. Múll.}

\section{Die Gantridneffe mit Dreserigem s)unde.}

H. testa umbilicata utrinque depressa: anfractibus obvolutis.

Linn. Syst. Nat. pag. 3534, Sp: 7 I

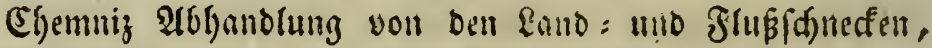
pag. IOI, Tab. I27. Fig. II 28 .

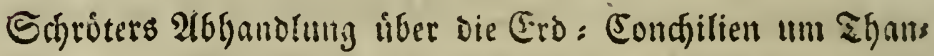
geffedt, pag. 194. Tab. 2. Fig. 24.

Gualtieri Index Test. Conch. Tab. 3. Fig. R.

Sd) lotterbed Acta Helvetica Vol. 5. Tab. 3. Fig. I6.

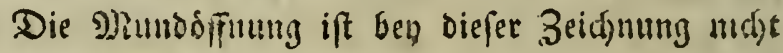
getrell uad) Der Natul abgebildet.

Müll. Hift. Verm。 pag. $27^{\prime}$ Nro. 229,

S) leje burd) ifre Mundoffunng fid') auffallend charafterifiretio croidnede wirb in unfret Biegent mur fparfam angetroffen, io, baß man Urfact)e hat, fie unter die Geltenlyeiten ju jählen.

Die Edale bat im Durd)idnitt 5 glarijer limien und in

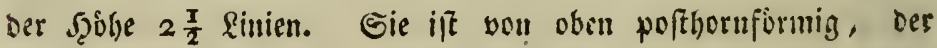
untere Theil if conver, yon Farbe sothlid)tram, einigermaltan Durdbidbeineno, fie hat feinen (Shanz, fondern if vielmelye matt, bartidalig, fein geftrelft unt tiberall mit felgr feinen meid):n

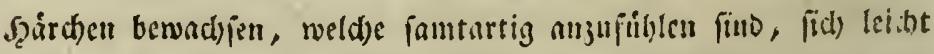
wegreiben uno Danu mit ber acrit ganz werchwinten. Ele hat

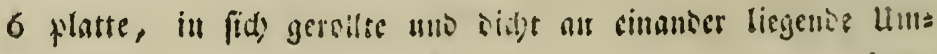




\section{$=65=$}

lăufe, weldse in Mittelpunft ein wenig eingeoricft fints. Der

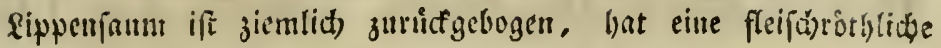
Farbe und bilot in feitum llmfange sin ftumpfez Drenect. Der

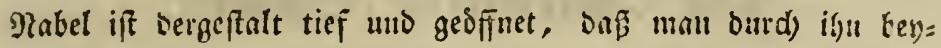

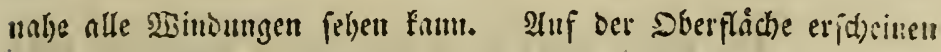

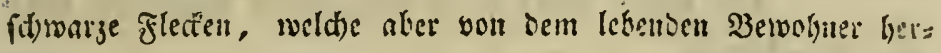
rúluren, butd) bie Sd)ale; bieje zcigen fid) bejonterz lebraft, wenn bie Eduede ins $\mathfrak{W a f f e r}$ gelegt wirb.

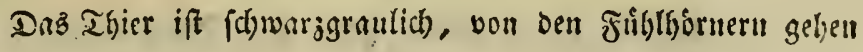

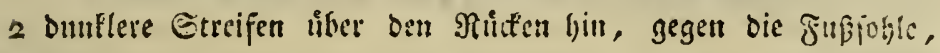

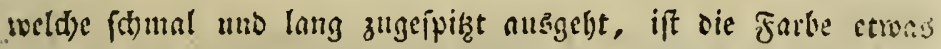
heller. Die Şant fiebt einer feinen zarten Ed)agringant gleid).

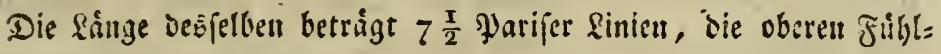
borner unefien $2 \frac{\pi}{4}$ Sinient.

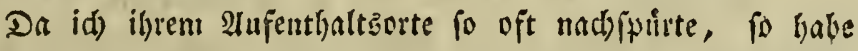
id) Dennod) zur Sommerbzeit nie ein (xemplar mit serf́chlofent:

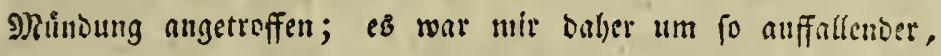

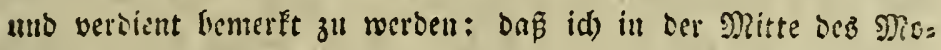

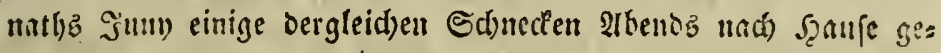
brad)t, an weld)en idy bie Mutroffifumg folgenden Norgen mit

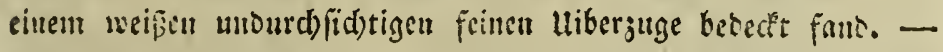

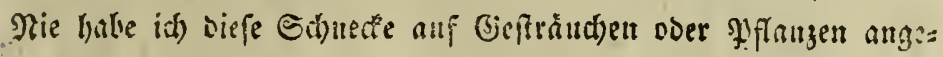

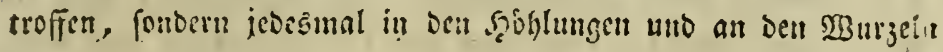

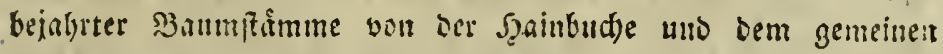

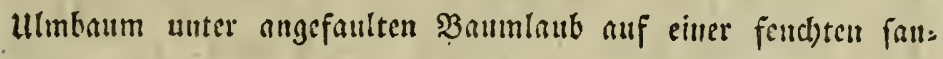

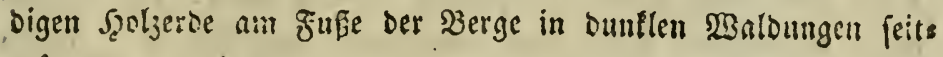

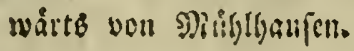




\section{$=66=$}

\section{Helix Crystallina. Miill. Tab. 6. Fig. 12.}

\section{Die cryftaulartige Sinnecte.}

H. testa perforata depressa candida nitida diaphana.

Linn. Syst. Nat. pag. 3635. Sp. 74.

Müller Hist. verm. pag. 23, Nro. 223.

S1 ogen ihren befonbern auffallenben (Eigenid)aften hat fie Şerr Eratsiati) Mitller bie crnftallartige Sd)nede genannt, weldhen Natymen fie auch in Der 'Flyat veroient.

Die (3rofipe ber Edjale hat in Durd)idnitt I $\frac{x}{2}$ झlarlier

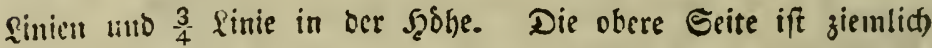
flach), unten ift fie runorr; fie ift febr bium uno zerbredilid),

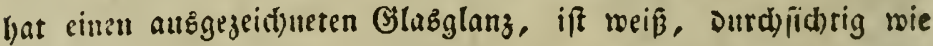

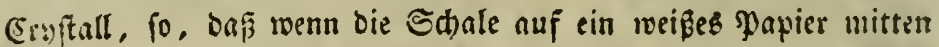
anf cinen fdumzen Rintenftrid) gelegt wiro, Diejer ourd bie

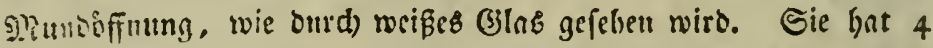

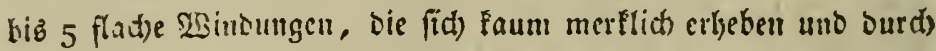
eine bentlidje Nath abgetheilt fïtb. Im skittelpuntt fteht ein

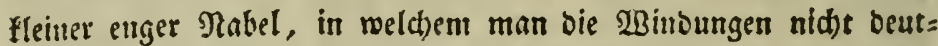
(ï) bemerten Fun. Die Mundoffnung ift halbmondformig, die Sippe hat Eeinen Saum, inwendig bemerlt' man, Dab fie von einem fehr suinten feiftenranto ober 23 ulf eingefafft ift. Durd) bie glabhelle Schale (d)cint ser $\mathfrak{B e w o h n e r}$ hody/f Deutlid) ourd),

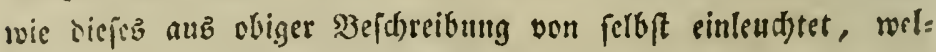

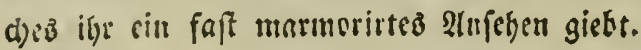




\section{$=67=$}

Das Zlituruen lyat bie finge von $\mathbf{I} \frac{\mathrm{T}}{2}$ gyarifer finien und

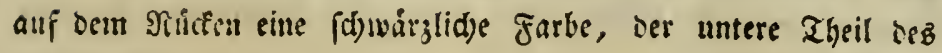

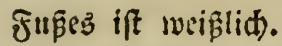

Dicie Epeciez finbet man in unfrer Begeno fegr felten, hins ter bem 2t6!aß an einem \&ed) fanal unter jerbrodenem Edjili uno

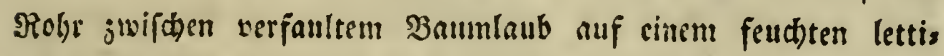
gen Boien iff fie von mir gefunden worbent.

\section{Helix Fruticum. Müll.

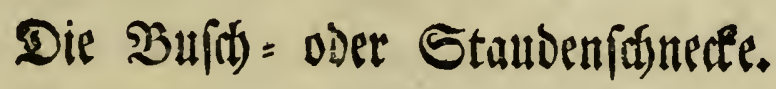

elabiata.

H. testa globosa umbilicata: apertura

Linn. Syst. Nat. pag. 3635. Sp. 77.

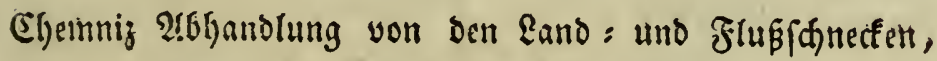
pag. 150. Tab. I33. Fig. 1203.

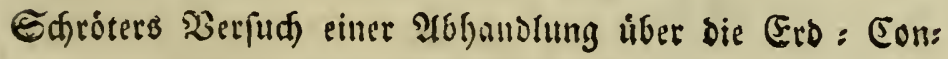
ciliell, pag. 178. Fig. 19.

$\mathfrak{B}_{\mathrm{on}}$ (iid) 3 Barietiteti.

Bon ber erften ift bie leere Sdale ganz weip. Mit bem Ibiere gelb, aud) weiß, mitunter am $\mathfrak{B}$ irtel blinlid), bin und wieber mit idwirglidisn flecten befprengt uno blafien

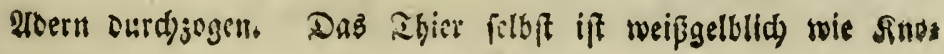




\section{$=68=$}

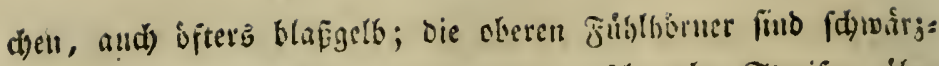
lidhgrau uno von biejen ziellen fidh 2 idhwad)e Streifen ulice ben Plliden hin.

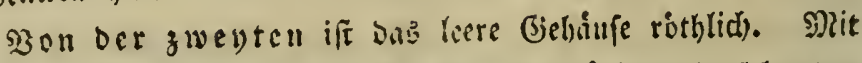
bem Thier ift bie grope witubung eben fo geförbt, bie folgencen gethen ins gelbe tiber uno haben braume Jlecten, Sfters ift aud) Der Gratmo fajwirglid)grau, mit gethen flectell uno syunften bes

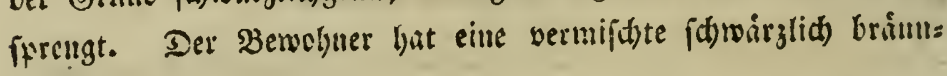
lid)e Farbe.

$\mathfrak{O}$ on ber oritten ift bie lecre Shale ebenfalls rothlia, aber etwab beller, mit ciner braumotheti Binbe, welde in ber

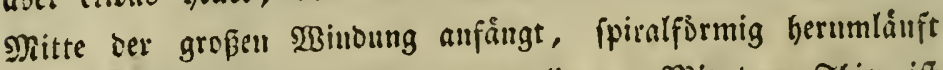
uno fid) gegen bie Endipitze zu verliert. Nit Dem Iljier iff fie getblict), unch) unten unb gegen bie গjotindung zu geht bie

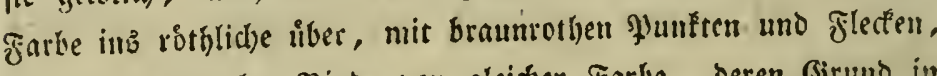
und ciner famalen Binbe von gleicher Farbe, beren Brrmo in Der Echale liegt, biciez ßand aus̈genommen, wech)eln die far: beu uno 3eidjunngen nit bem $\mathfrak{B} e$ wobner fel) mannigfaltig at. Die kraunrothe Binbe hat in Edhlunes eine eben fo lebhafte Far=

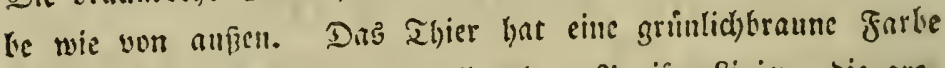
unt betringt it bet Ringe I 3oll unb 3 Syarifer finiell, sie gres fen Friblyorner meffen $3 \frac{x}{2}$ finien, biefe fino etwa ounfler und

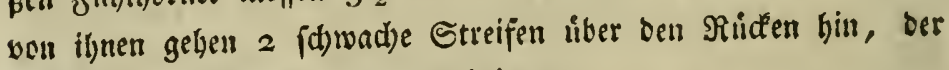
fuf geht abgeftumpft etwab fpiţig jut.

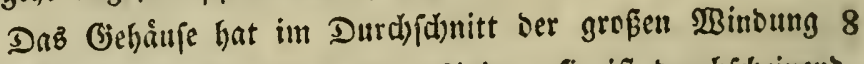

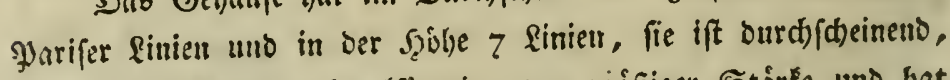

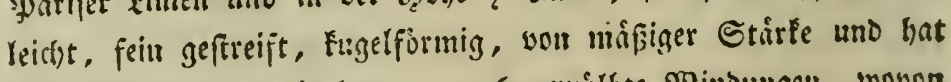
cimsn fertglanj. Sie hat $5-6$ geworthe MBinoungen, wanon Die erfte gres uno gleidy)ism wie aufgeblafen ift, uno in sine 


\section{$=6 \ddot{9}=$}

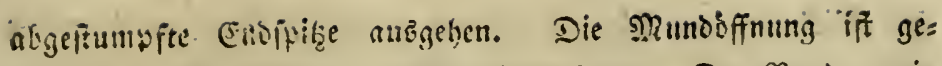
rumbet, balbmomoformig, olyne Sippenfaum. Der Nano wenig

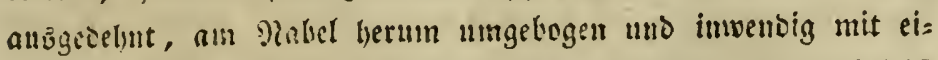

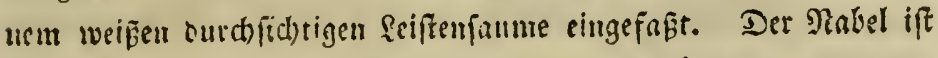
Effen und eng, unb ourd)bohrt onz ganze Bielgåupe.

Nan finber fie anf biebilihen, bejonderb auf Erlen, \&ign=

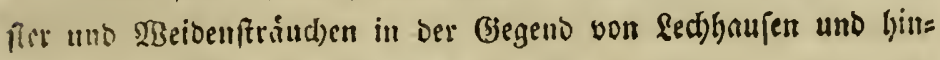
tcr bent 2tblap weiter am Sed) hinauf.

\section{Helix fylvestris. Mihi. Tab. 7. Fig. 13. Die Balbifinecte.}

ת Wh) hate biefe Sdurcte mit allen yorhanbenen Bepdreibungen Der Erojdnnecien feht forgfíltig vergliden, allein burd)ant feine Derjelben ganz genuigend gefunben, weldye ben Charafter ber vos: liegenden genau uno paffent beftimmt båtte, mešwegen ez unir mahl erlanbt fdjien, ihr eitren eignen Pabmen zu getien, ull: weit ich fie einzig und allein in 20 aloungen angetreffen habe, fo gab mir ibs 2(ujentlyalt Beranlaffung, biefelbc Helix fylvestris zu benemmen.

Die Brope oer ed)ale betringt in Der Breite $\frac{\mathrm{T}}{2}$ 3oll uno

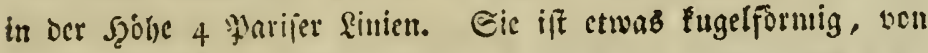

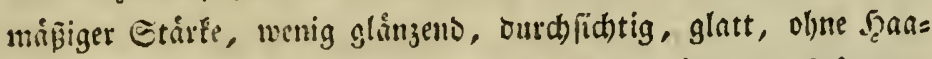
re, son Farbe gelblid)braun, weld)e in Der Mlife ber Minkung

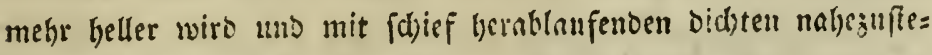
benten feinen Etreifent bejeidunt. Faft un bie Mitte ser el= 


\section{$=70=$}

ften Sinbung legt fid) eine idmale weißlide Binte, welde felis blas iff uno fid) beym zwenten (Beminde fidon mieber verlicrt. Shre Edjale hat $5-6$ erlhabene umgange, wovon ber erfte grổ uno laaudjig ift uno fid) in eine frumpfe Epizze enbigen. Die Rumboffnumg ift zugerunbet, halbmonoformig; der Rano etwas erweitert obue sippenfaum, an ocm 9?abel menig burnifs

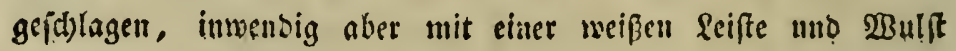
eingefaß̧t. Der Nabel ift offen uno geht bis jur Enofpitze, itt weld)er man cine, mand(b)al aud) zwey) 2 Sindoungen bemerken famu. Das Thier gat eine fornige Szaut, ift roftgelblid), mit

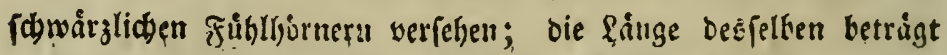
7. Farifer finien, die oberen frubllyorner fino $I \frac{T}{2}$, finie lang. Mit Denz lebendigen Ibjer fiefte bie Єđale bråmulidigran aub,

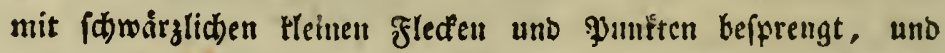
an ber groben 2 Binbung ziebt fid) eine breite weiplid)gelbe aloer herum.

Man finder ije in ben tiefifen faublyolzmaloungen unter

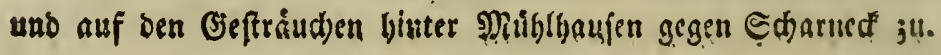




\section{$=7 \cdot 1=$}

Helix aculeata. Muill. Tab. 7. Fig. I4.

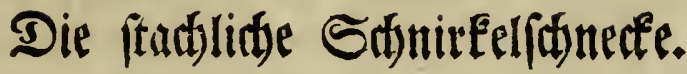

$H$. testa trochiforme umbilicata untrique convexa fusca: costis aculeatis, labro albido.

Linn. Syst. Nat. pag. 3638. Sp. 90.

Chenmiz 2 bhandlung von ben \&and: und flupifinecten, pag. 153. Fig. 1209.

Der Naturforfher 19. Stüff, pag. 165. Tab. 2. Fig.

1. 2. 3.

Müll. Hist. Verm. pag. 81. Nro. 279.

Diêe Edjnetfe ift nidjt nur bebrmegen, weil fie fehr flein uno im 2 lufüud)en leid)t biberfehen wiro, felten anzutreffen, fons Dern an fid in unfrer (Jegeno áuperft rabr; aud) bave id fie in obenerwáinnter. 216handlung und in Naturforfder fo unridytig abgebildet gefuntoen, Das id mid) ketwogen felle, foldhe fier gee treu unad der Natur gezeidynet und aud) im vergríperten Miaa: fabe aufjufithren.

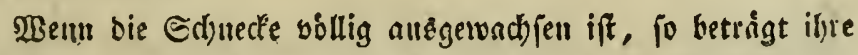

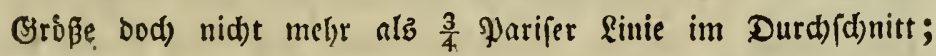

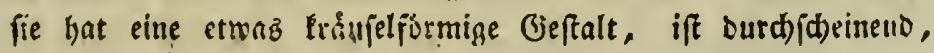
perr feinfdalig, bráunlid), uno auf oer ganzen Dberflád)e laufen

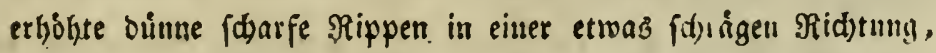

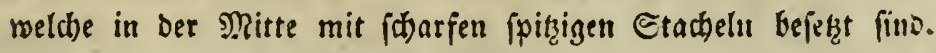
Man jáblt an igr 4 ergabene gevilbte $2 B$ indungen, weld)e fart 


\section{$=32=$}

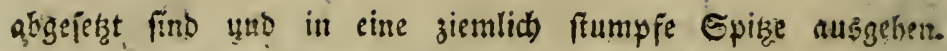
Die Rumbsfinting ift rutublid, faft monoformig, nit eincum

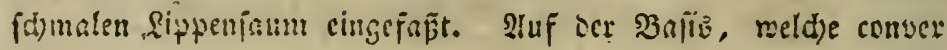
if, ffeltt ein fleiner enger Pabel, weldyer aber boch biz zur Enọipize himreid)t.

Das. Thietd)en if auf bem Sinpf und feitwarts am Szalie

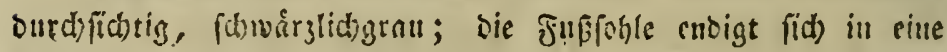
fring Spitze umb bat cine weiplide Farbe. Die oheren fliflfors

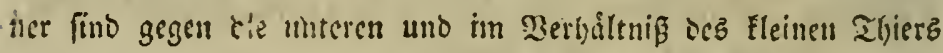
jremlid) lang, anj beren Enoppitien man bie fidwarzen 2 lngen wie foine \$ु)

Shren It:ifentgalt cutbectete id) in ber Bjegend yon Eteşling

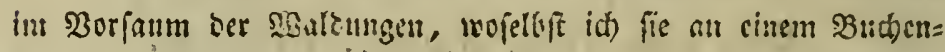

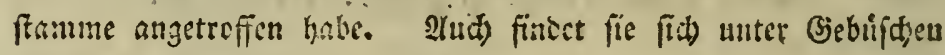
unter angefaultem $\mathfrak{B}$ anmlatibe.

\section{Helix lucida. Mihi. Tab. 8. Fig. 15. Die burdofidtige Paberfornerfe.}

भI

ud) bey bes genauffen Dergleidung mit allen sorbantenen Befdreribungen scutid)er (Coudjilicu wurbe atd) feine cinjige vout

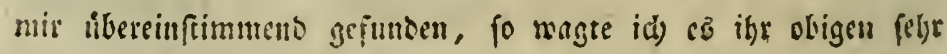
pafferten Stalmen besjulegen.

Dic Edale bat in Durdimeffer 3 frarifer sinien und in

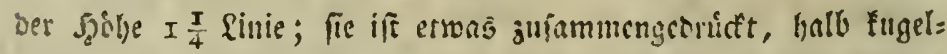

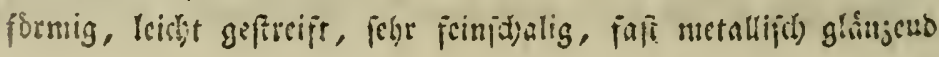




\section{$=73=$}

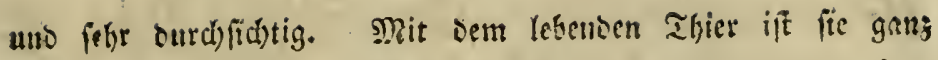

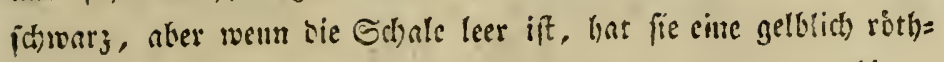
licte Garbe mit einer weipliden Enofpitze. Sie hat 4 bis 5

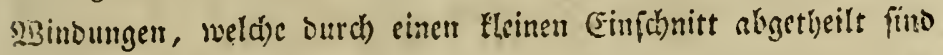
uno fid in ftumpfe Epise entiget. Die smuboffum, if ges rutibet, balfmonoformig, wenig erweitert, ganz oline sippens faum; ber obere Ecitemrant feefyt etwas hervor. 2tuf ber un= tern Eeite, weld)e etwas gewolft iff, finbet fith ein offiner und

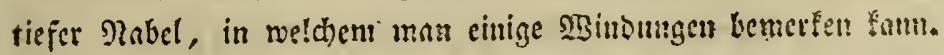

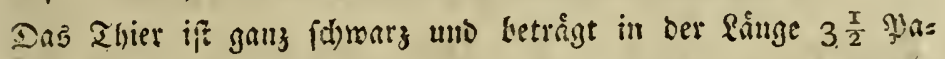

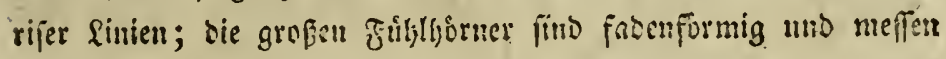

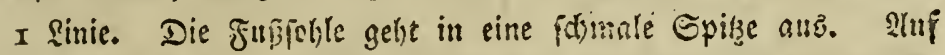
ocr Sutpfertafel ift sie leele Sdalc mif beysen Esiten siggebil= fet, um fie befro beffer feune? ju lernen.

Sie if in unfre: (orgeno nicht felten, id) hate fie an cis nem fech) Fanal binter bem Siblaß unter angefaulten Şaumblat:

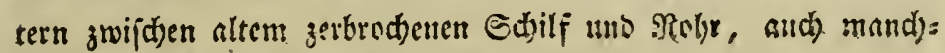
mal an angejaultem forts gefunben. 


\section{$\leq 74=$}

\section{Helix fascicularis. Geoffroy. Tab. 8 . Fig. 16.}

\section{Der Feberfuiftringer.}

H. testa umbilicata pellucida: anfractibus tribus canaliculo discretis, apertura orbiculari non marginata.

- Linn. Syst. Nat. pag. 364I. Sp. I 85.

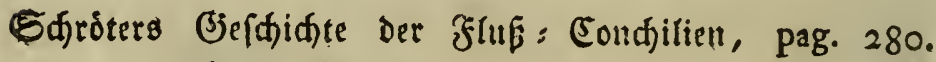
Tab. 6. Fig. II,

Beoffroy 2tbhandlung von Den Eondilien, welde um $\mathfrak{Y}_{a}$ ris gefunden werben, pag. I02.

ఫ̧udjs Ephenterioen I, Қanlo I. Şeft, pag. 54. Tab. I. Fig. I.

Milller Hist. Vermium, pag. I72. Nro. 358. Nerita piscinalis.

巨 owolfl in Linn. Syst. Nat. alz in હd)roterz Biefdidte ber FruF = (Sondbilien, in weldjen letzeren sod) bennake jeseżmal auf

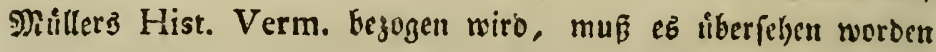
fenn, dieje son Miller unter oem Mnlymen Nerita piscinalis aufgefilibrte Ed)necte zu citicen. Gimelia bat fie zwar alz Helix piscinalis aufgeftibrt, fie ift aber Ecinesinegb vout biffer vers idjieben, fonbern bendoe fint cine und bieferbe Epeciez.

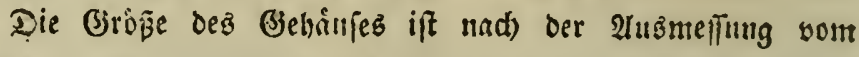
Btunde an ber \$Růnbung biz zur Enofpitze 3 Yarifer fimien 


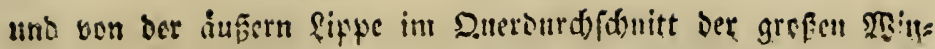
bung aud) 3 finticu. Eie hat eine frinfelformige etme fugel=

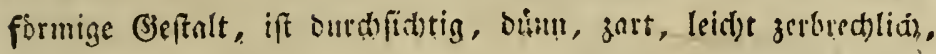
mit felfe feinen Streifin ser finge lad) verfelhen utlo won gelts.

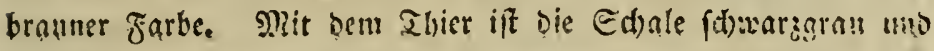

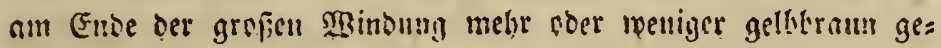
flect, Sie bat 4 ranse Wilnoungen, wouen eine siber bie alls

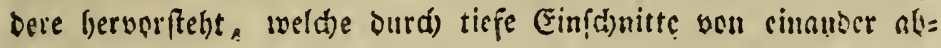
gefondert fint und in eime abgeftumpfte Eptitze ausgebcu. Der uncere Dheil bilbet gegen bie Mitte cinen tiejen staiel, weld)ęr

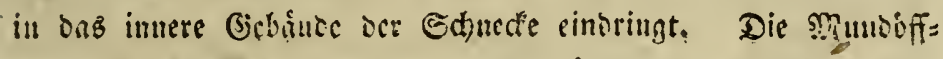

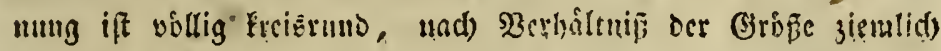

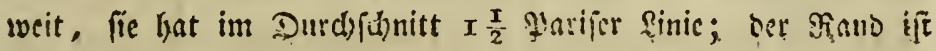

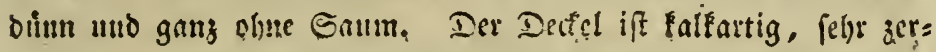
bred)lid, yon auken etwak concav, in Mittelpunţt bat er eine Bertiefung in oer Birobe eituce fleinch Shadelfmopfes. meld;e nit zarten berticften \&inicu umgeben ift. Das Thler hat eine ourdefid:= tige graue Farbe uns firedt antere feinen 2 fabenformigen griblhor:

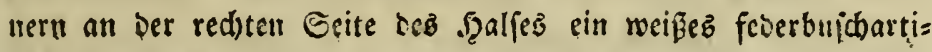
ges Drgan hervor, an weld)en ber Gdart oer feder anf hey = ben Eeiten wellenformige \$Bimpern bat; *) alner biefen 2

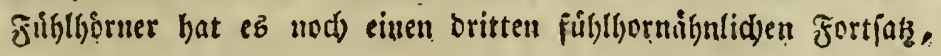

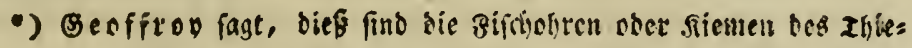

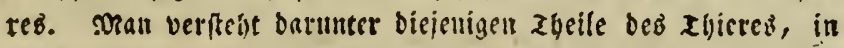

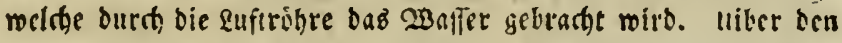

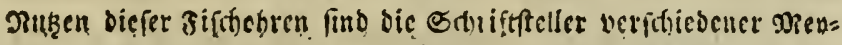

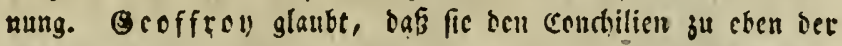

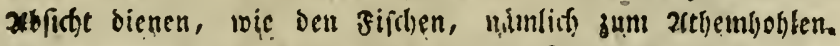

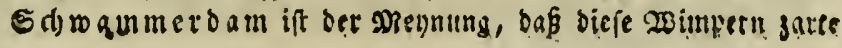




\section{$=7^{6}=$}

weldher etwab lánger unt jürter als bie 2 anberen ift uno nid)t vorme am Şalfe, fondern aub oer Geite hervorgeht; Dẩ Thier

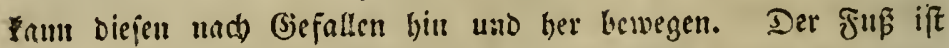
ganz nugernndet uno wiro nad bintenju biciter, wo an ber cbe: ren Eeite ber Dectel beveftiget iff; vorme theilt or fich in 2

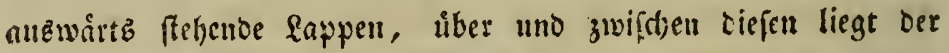
fropf, weld)er eine cylinberformige Beftals bat. Die fdywar

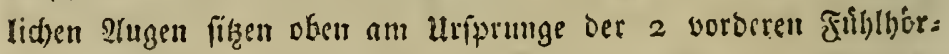

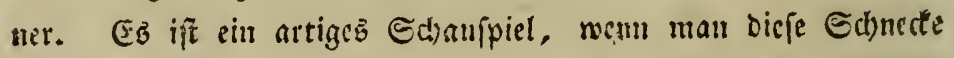

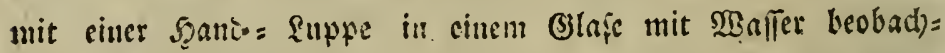
ket, wic ez ben Declel anfiscbt uno im Syeransfriedyen feituen

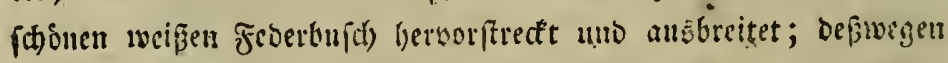

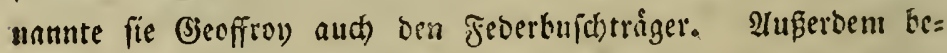

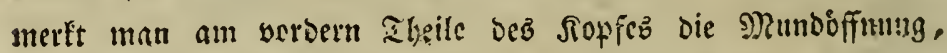
eine fleme lángliche perpenticuline Seffnung, sie wegem sem

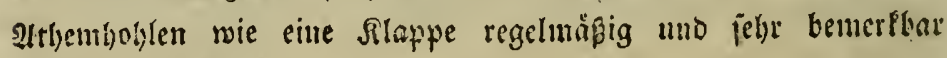
auf utto zu geht.

Diefe Edmecte Darf mit Valvata cristata,' Ed)rank Faut-

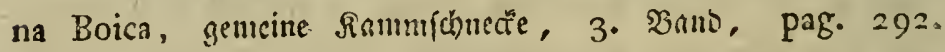
nid)t scrwedifelt werben; bem iene ift ein glefthorn unb biefe hat einen Erauflformigen Ban; beßwegen hat ber verbienftudle

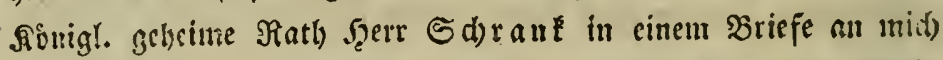
vorgef(d)lagen, bie Valvata cristata nad) Mitler und bie Helix fascicularis nach (seofiroy zu hẹeunen, weld)ez id. hiemit un

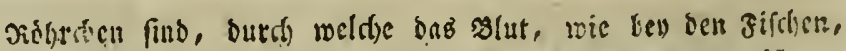
ungetrieben mitd. anatint fagt: Shre seftimmung ift un=

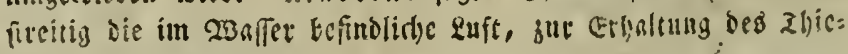
TCB., aEgufontern. 


\section{$=77=$}

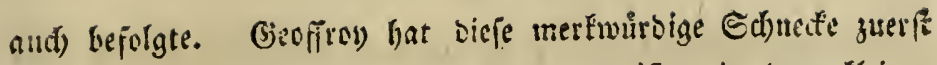
entoectit und befdricben, unb fie am meiften in bein Eleinets Fluffe Bievre in jrantreid) gefunden.

Ju ber Giegeno von 2luggburg wiro fie an verfdelebenent Srten utro zmar nid)t fparfam angetroffet. Nian fittoet fie in

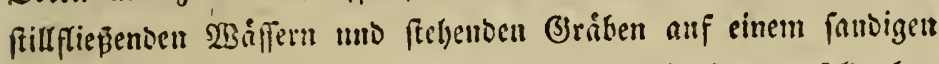

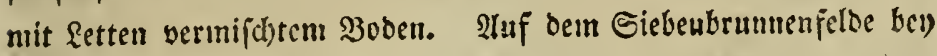
Şaunferten aber bate id) fie anf einem (d)lammigen lettigen

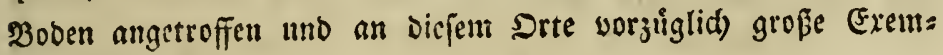
plare erbaltert.

\section{Helix bidentata. Chemniz. Tab. 9.}

\section{Fig. 17 .}

\section{Daz. Rnopfgen ober Der zwiefad gegafnte Rráufel.}

H. testa pyramidale fubcarinata fasciata fubtilissime striata, labro bidentato reflexo.

Linn. Syst. Nat. pag. 3642. Sp. 231.

Ehemuiz Condjilien: Eabitret 9. Saand, pag. 50. Tab. 122. Fig. 1052.

Serr Paftor Ehemniz fagt in feirem Eondjilien = Eabinst pag. 50. ber Şerr Superintenbent Sd)rober habe ilm biefe ar= tige fráujefformige Schnecle mitgetheilt uno if)m baben gemeloet: 


\section{$=78=$}

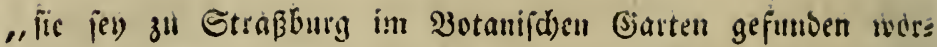

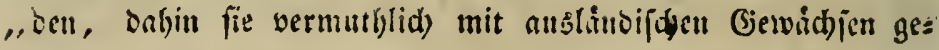
"(rradt worden peyse."

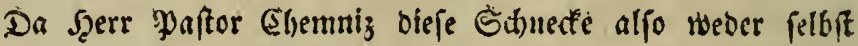
gefunten, nod) viel weniger ibr. Baterlaito anjugeben musre,

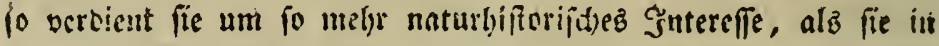

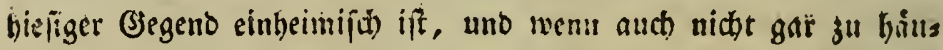

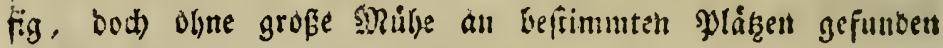

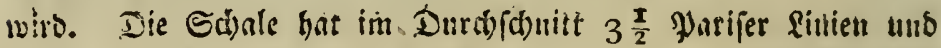

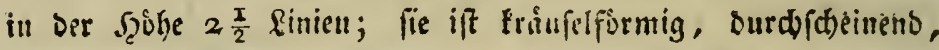

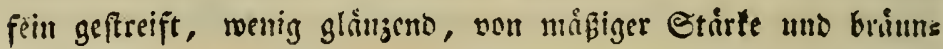
lid) von Farbe, init einer blaffen weißflichen fdmalen Binse um:

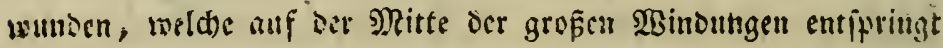
unb fpiralformig an ben Einjonitten Derielben fortlauft. Cie

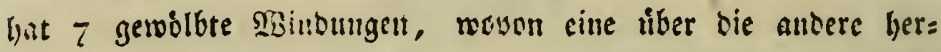
vorficht und welche it eine abgeftumpfte Epitge angelgelyen. Die

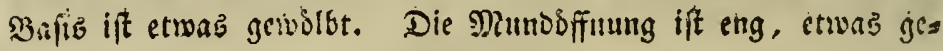
Erủft, nach ausen wenig erweitert; fie ift mit einem folmialen Sippenjaum umgelber, welcher eine róthlickmeiffe Farbe bat; an

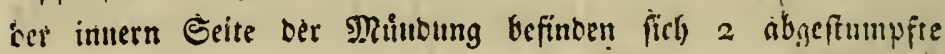

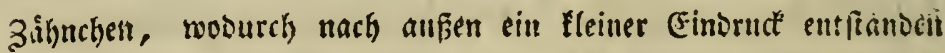

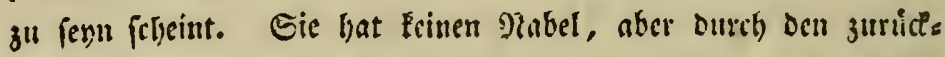
gelegten Sippenjaum entfrel)t ein Nabelpunft. TSenn caz Thier noch in Der Echale lebt, fo ijt die garbe anf sem shirbel ount: ler und auf ber grop̃en Wzinoumg zeigen fich frbwarze, aus

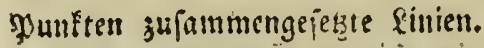

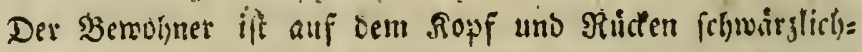
gran, mit 2 Etreifin von ben folblthomern biż zum Sinutel

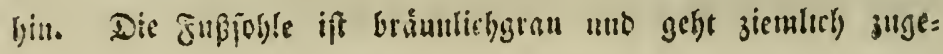




\section{$=79=$}

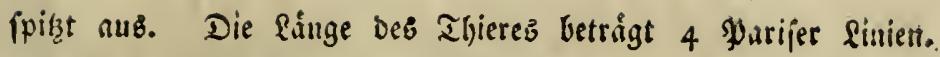

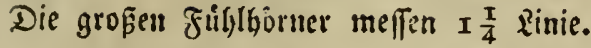

Man finbet fie an frlyattigen Srtell unter Bjeblifchen auf Der Eroe, wetu fie nicht zll bicht mit Syflanzen bewackfen ift, in Borbolzern ber $2 B a l$ ungen in ber Giegento won Eteşling uno Fricoberg, eine Ermose yon Inggburg.

\section{Helix Cobresiana. Mihi. Tab. 9. Fig. I8.}

\section{Die Cotrenifte Sdunirelifinecte.}

Jud) Ephemerioen I, Band I. Şeft, pag. 56. Tab. I. Fig. 2.

So reid)haltig aud) bie Sammlung ber Eondjillen = 2Berle

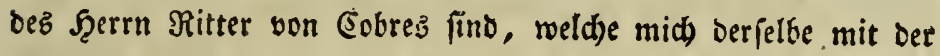
liberalfen 3uvorfommentheit benukgen ließ̧, fo fano id weber in Diejem reidjen Sdakge, nod) in meiner eignen fitteratur bief Sd)uede meder befdrieben, nod) abgebilbet; zwar fülyrt Şert Paftor Elyenuniz im 9. Baube feinez (Sondilieu = Cabimetz aud) eine Helix bidentata auf, meldje aber in unfrer Giegend einhfi: mifd ift unb mit ber bier von mir bejd)riebenen viele 2rebnlid), feit hat; ba fid aber biefe gegett jene baburd wefentlid und unverfennbar unterídcibet, Daß fie beftimmt nur einen 3abu hat uno fich Durch bie gelblid)e Eiufaffung an ber Múndung vor: अiglid) ansizeid)net, mithin - wie melyere Dutzento vor mit liegente Exemplare beweifen, Peine Epielart, foniern diefe હd) 


\section{$=80=$}

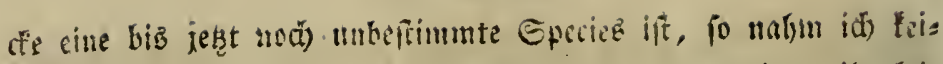

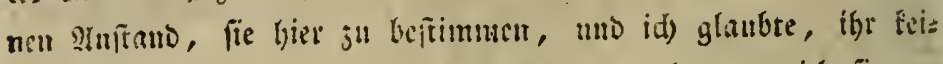
uru chrchsollern Pabment grten zu fismat, als wenn id) fie Dem, um die ge[ammte Platurgeidsid)te, ingtefondere aber unt

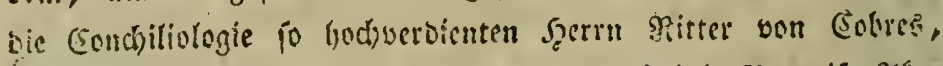

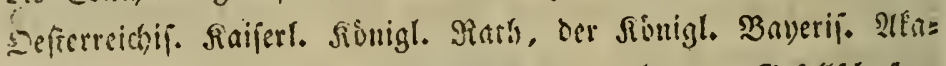

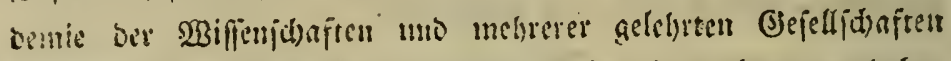

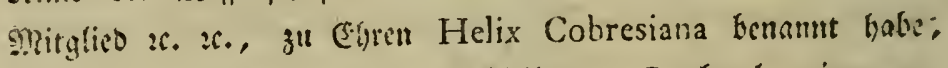


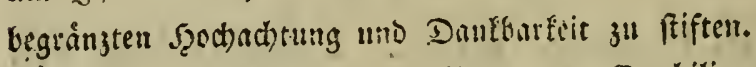

Pun bie nâhere Befdrcibung ocr Eond)ilie felbft: Dicfe

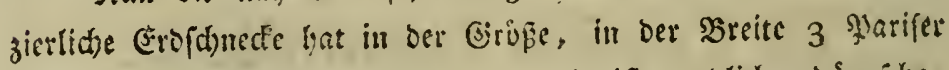

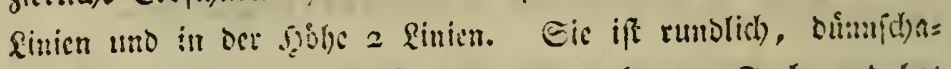

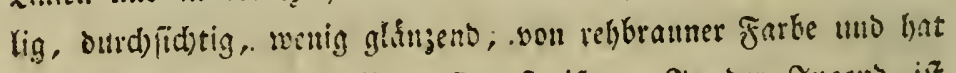

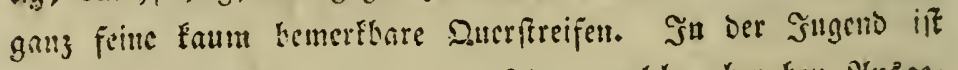

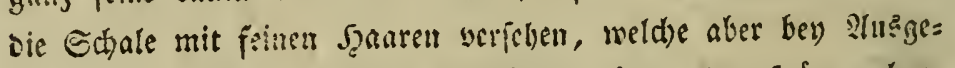

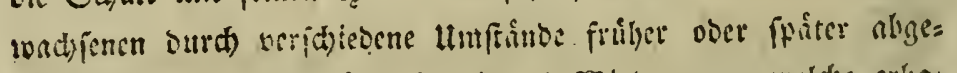
ricben werben. Sith juble

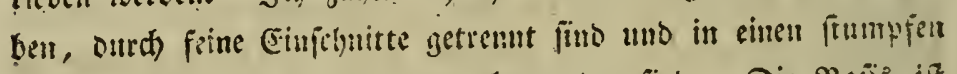

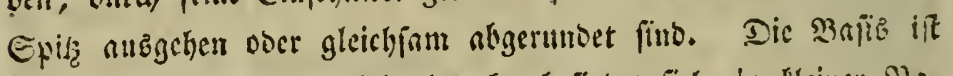

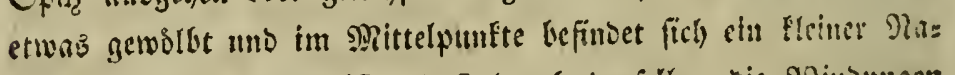

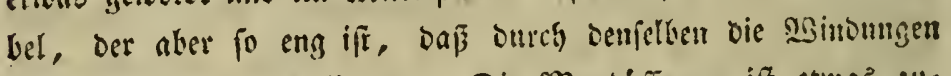
nicht gejelyen werben formen. Die Mumboffinung ift ctwab zu= faumengeorictit, faft halbmoniformig mo liegt in ocr Ducre,

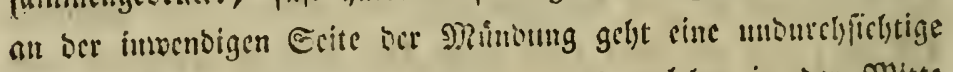

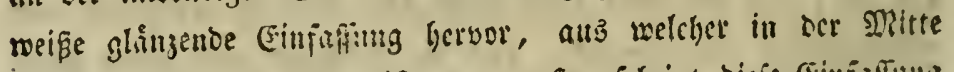

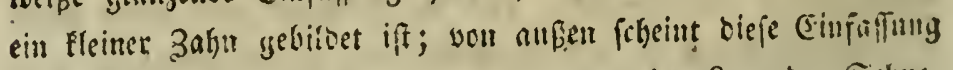
bentlich ourch bie Ecijale, de aber wegen ber fage der Echne=

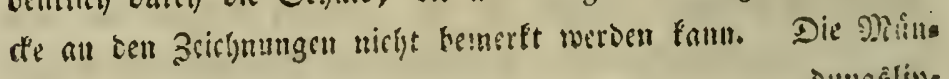
Dumbâlips 


\section{$=8 \mathrm{I}=$}

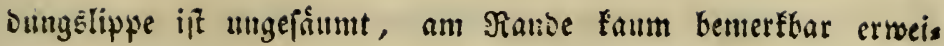
tert, gegen ben Nabel bin aber felfr wenig zuríff́gebogen. Llm Die Ritte Der erften SBindung láuft cine fd)male, hlaß̧e Binve Die fidf aber bey Der zwenten vertiert. Dna Thjer hat auf Dem

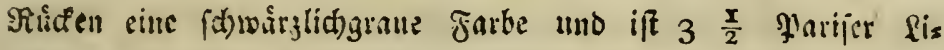
nien lang, bie obern Fúblhorner fino you berjelben Farbe uns

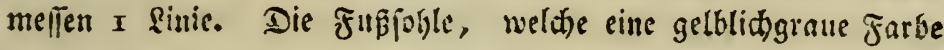

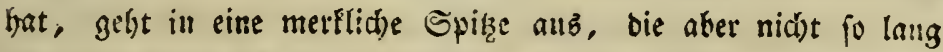

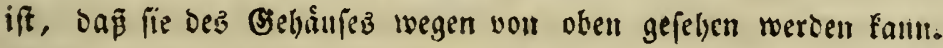
SBenn Der $\mathfrak{B} e$ wolkter nod) in ber Sabale lebt, fo ift fie bunfler uLb mand)mal bin uno wieber mit idharzen \$ounten uno gel= bell Streifen gepợbt.

Man findet fie unter (jishifaten, welde nid)t ju nabe am

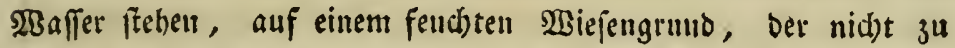
bidat mit Ylanjen berwadjen ift, in ber Giegend yon Redhganfen und binter bem 2lblaß̣.

\section{Helix limacina. Mihi。 Tab. IO. Fig. 19.}

\section{Die Galbbeberfte Erdiftrecte mit 3 $2 B$ in= Dungen.}

(3) aneinanberreigt und finfenweife von cinanber cutfernt, fimbet

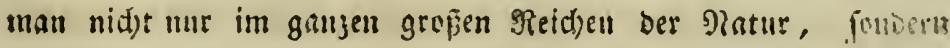
aud) in ifreu einzelnen 2iththeilungen; ber Berfafier, ier ez

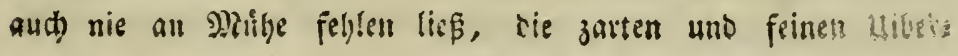




\section{$=82=$}

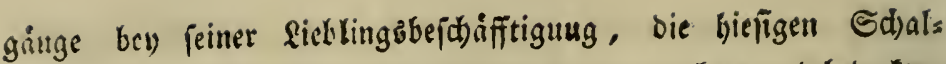
thiere aufjufuden, fand biejeb anch an ben nadeten uno bededten: Cünecten vollfommen beftáttigt, diejez gab (jelegentjeit bey zwen in ber Biegent 2/ugêburg aufgefundenen Sd)necten, um felbit bie Mliberginge fraftig mitzubezeichnen, bie eine Helix limacina, bic antore Helix limacoides ău nemuen, 'iente nás Gert fid) mehr einer Limax, biefe melyr eitre Helix.

Das Ibict darafterifitrt fid) nuf eine bewunberungsmits Dige WSeife, uns ift unftreitig bie Mittelgattung unb ber feinfte llivergang juifden ben Limaceen uno Heliceen uno follten

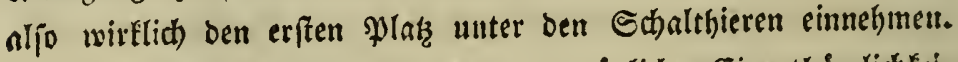

Die Shale felbft hat leime yorzhiglid)e Eigentlyủmlidfele ten, nur biep verbient an ifr bemerlft. zu werben, oñ fie

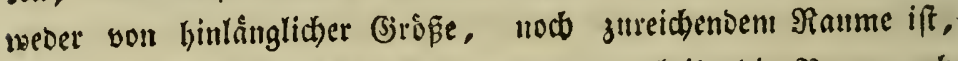
um bie ganje Edurede zu faffen, baher gab ifr bie शarur uod) einen befondern Edbilo, um igr bie benothigte Bebectung zu ver: fidaffer.

S.b) will num eine genauere Bejd)reibung Diefer Helix limacina verjuchen.

Die vollfoinnent anbुgewadjeme Sd)ale hat in ber Range $2 \frac{T}{2}$

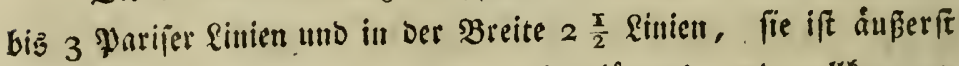
zart uno feii, febr zerbrectlid), glasglánzeno uno volffommen burd)fithtig, glatt, gainjlid) olye Streifen uno von fold)er feid): tigkeit, bẩ man fie mit einem Şaud wegblafen fann und 6 Ettid nur $\frac{x}{2}$ (Jran wiegen. Shre Sdale ủbertrifft unftreitig au Seidytigfeit uno Feinheit, (Slan\} uno Durd)fid)tigleit alle librigen Sirten son (Erbichuecten. Ilyre Bnuart ift flad), niebergebrict,

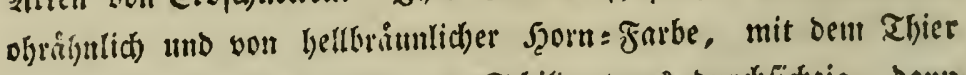

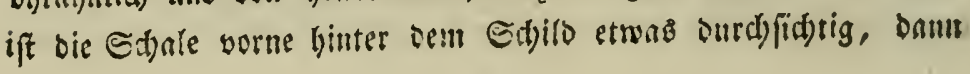




\section{$=83=$}

folgt ein gelblichbriutulid)er glect, welcher fid) linglid in ser Quere liker bie Echale ausbreitet, uns ber tibrige Theil bat eitte fidom,

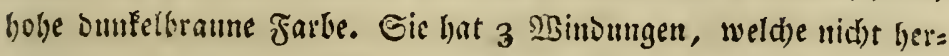
sorfelyen, fondern wlatt an ber Geite liegen unb Durd) einen

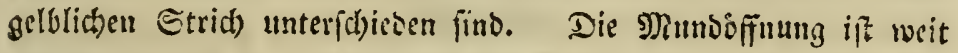
und breitet fid) zur linfen Geite bogenformig aus. Sie ift mely: lang als breit, fo Da pie fie ein volltommencs sual bilout.

Die Rippe ber sRunoung if vorne idharf, wenig ectrig,

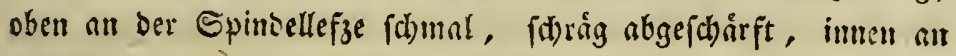

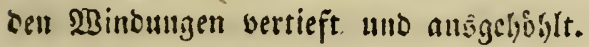

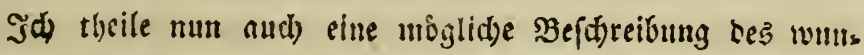

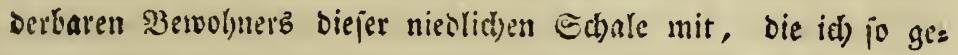

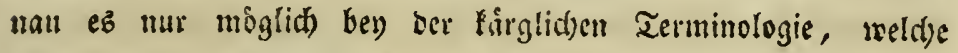
wit uber biefe Giegen(tintbe hahen, mad)en fam.

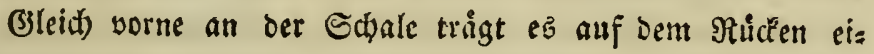
nen glåmjenten, rumjlichen Mantel ober Sdhilo, weid)er fobwair

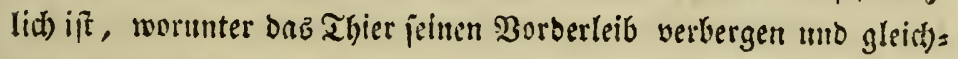

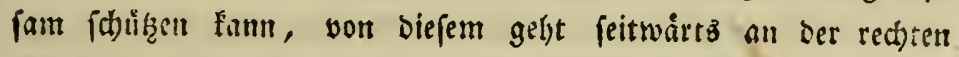
Seite cin verlingerter 2tuhgung, unter weld)em fid) bie Suftroige befindet unb legt fich von aufen am Enbe halb fugelformig an

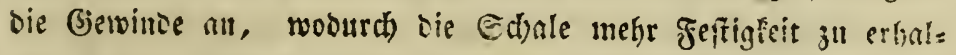

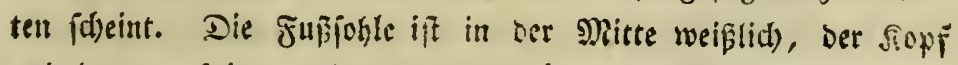
utto Der zugeipizzte Şintertlyeil (d)wirglid)grau, unten auf be:

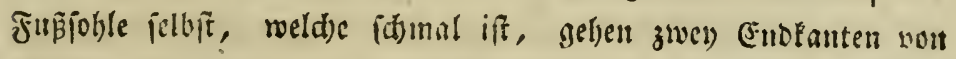
gleicher Farbe voin Sopf biz jul Endipitze binunter. Sie. laat

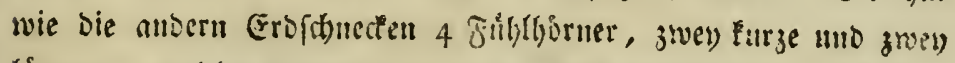

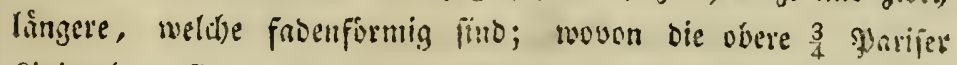

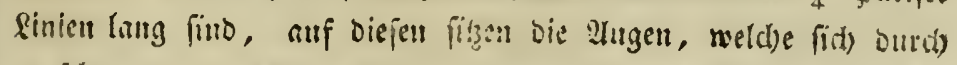

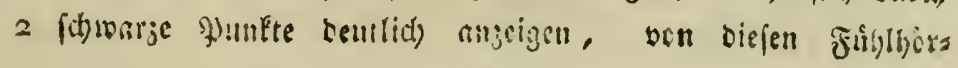




\section{$=84=$}

nern ziebsen fid) 2 (d)wárzlid)e Streifen, bis zum Mantel bin, zrwifhen biejen befinten fidh vor ber Etime 2 anbere fabåd)ere iffulid)e Etreifen, welche furger find und zufammen gleid) fam einen lángliden Fled bilbent. Die Entrofize ift fd)mal, lang,

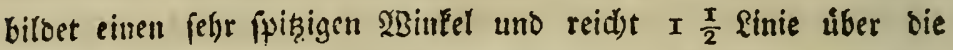

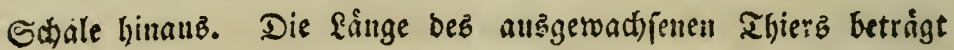

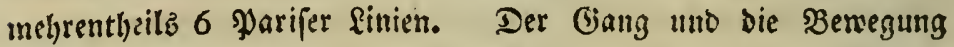
ift munter und lekhaft, balo ziegt eb bent Sopf und bie frihls

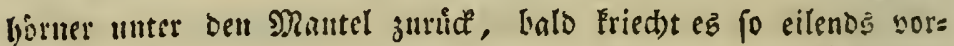

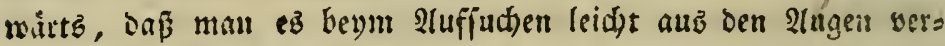
lieren $\mathfrak{f a n n}$, weld)ez, wie befannt, bey anbern Sd)nețen nidjt leid)t ser fall ifr. Eie pflanjt fid) eben fo fort wie sie anbern

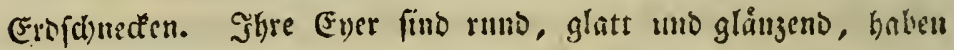

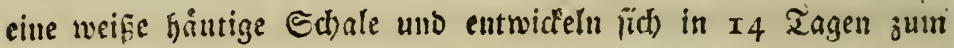

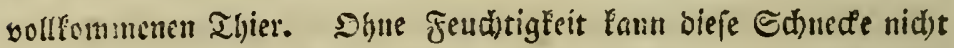
lange anzbauern, fie if fo zårtlich), baß̧ fie an trodfentu Dr= ten im friecthen antlekt und bald Darnuf firbt.

Der 2tufcuthalt biefer neuen હdynefengattung wurte von mir mit bcu lebbafteften Bergnigen an ber Schmutter, einem

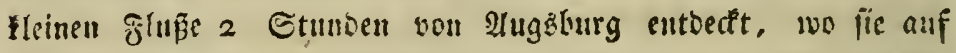
eiurm gylakge in zablreicher Nenge son mir angetroffen mutre. Eie wobut in Gejellidaft mit ber Helix limacoides jomifdsen Den Epalten, Bertiefungen uno Şóflungen oer Eroe, unter zartem Strab unb Moos anf eirrer guten abet fantigen NBicjen= erbe. 


\section{$=85=$}

\section{Helix limacoides. Mihi. Tab. II. Fig.20.}

\section{Die balbbederfte Erofdinecte mit 4 Win= bungen.}

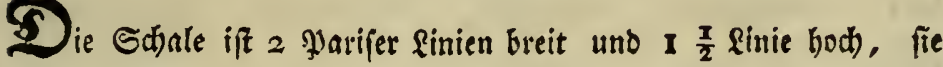
ift gelbgruinlid), burd)fidtig, glatt, !eidht geftreift, fehr bimue und jerbred)lid. Mit bem Thier hat fie eine ropttoranne Farke

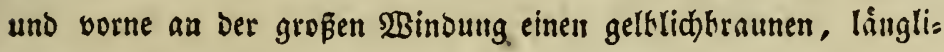
d)en fled. Şgr åufere form fommt ber Helix limacina felyr nabe, nur ift fie mehr rundlich und gewolbt.

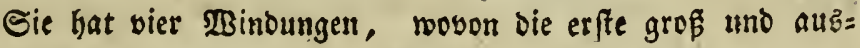
gebreitet ift, bie übrigen fino llein, zujammengerollt, erbeben (iich. nur fehr wenig ifer bie Doerflád)e uno liegen etmas zur Seite. Die Munbofffung ift grof uno oval, bie Minoungb= lippe iharf, an ber Epindellefze mit einem felyr fhmalen,

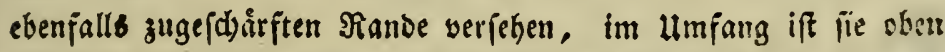
eben fo breit als unten. Das Thier felbft erreicht nidjt ganj bie Grobe Der Helix Limacina uno hat eine rotheraume Jarte,

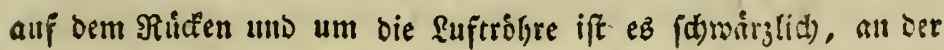

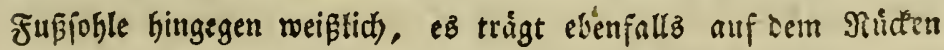
einen Mantel yon eifengrauer Farbe mit Runjeln, welder ben Şals bebectt, nur ift er etwas turger, uno von biejem gefte cin ebenfallz etwas verlángerter 2lnhang aub, scr fid) aup̧cti an die Creminde ber Echale anlegt.

Sie hat mit ber vorigen einerley 2tufenthalt. 


\section{$=86=$ \\ Helix vivipara. Linn.

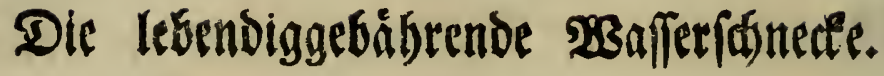

H. testa impervorata fubovata obtusa cornea: cingulis fuscatis, apertura fuborbiculari.

Linn. Syst. Nat. pag. 3646, Sp. 105.

Lister Exercit. Anatom. Tab. 2, Fig. I -5

Gualtieri Index Test. Conch. Tab. 5. Fig: A.

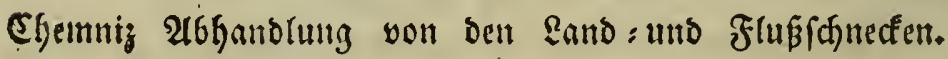
pag. 136. Tab. 132. Fig. I I82- I 185 .

Sturn Deutfhlands Fauna 6. A6theilung. Die Wrurmer.

2 Қ̧eft bas II, Siupfer.

Siefe Sdunede hat ifren Ramen lebenbiggebährento baher ers balten, weil fie nicht wie andere ihres (bejhlectits erft Evyer legt,

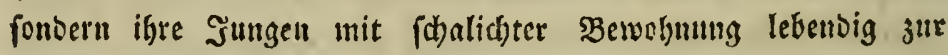

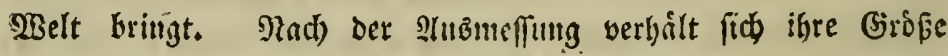

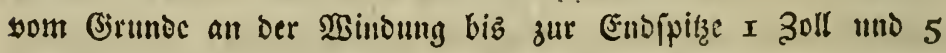
Sinien uno von ber anßern Sippe in Duerourd/4hnitt ber großßn Binoung I $30 \mathfrak{l l}$ a finien Warifer Naap. Eie bat einen fu= gelformigen juge(pitz̧ten $\mathfrak{B a u}$, ift buum, jiemlich) Durd)fid)tig, menig glänzento uno mit feinen, bidhten, längliden Etreifen

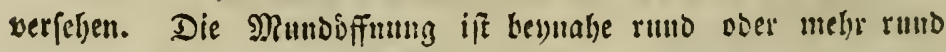
alş oval, innentig blaulid), die Rinnsungelippe, meld)e büin 


\section{$=87=$}

uno idarf iff, legt fid) neben ber Epindel an, weld): bis auf bie

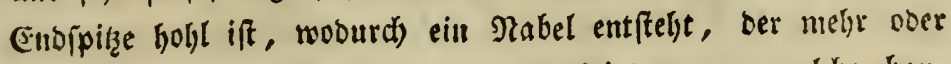
weniger offen iff. Sic hat 5 biģ 6 SBinoungen, welche banl= d)ig, ourd) ftarke Bertiefungen getrennt finb und fid) in einen fpişigen Punkt endigen. Die (Strunb farbe ift (d)wårglid)fraun *)

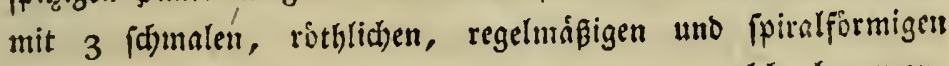

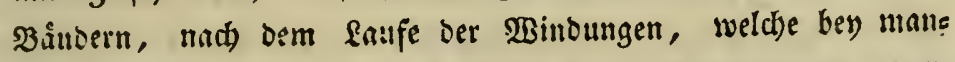
den Sollbivibuen gar nid)t zu fehen fino, an anbern nut gegen sas fid)t gehalten, fdwath ourdjidhimmern. Der Dectel ift borns artig, mujhlid), bieglam, braunroth, burdbfid)tig, mit concells trifhen Streifen uno feitwairtz son außen mit einer Bertiefung, weld)e auf oer inwendigen Seite eine Erboffung bilbet, wo er nut ber Ferfe bes Fupez hefeftiget ift. Der Bewobner ift nieos

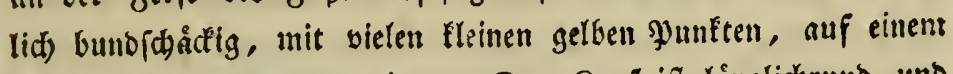
braunen farbengrund geztert. Der Sopf ift linglid)rumb und

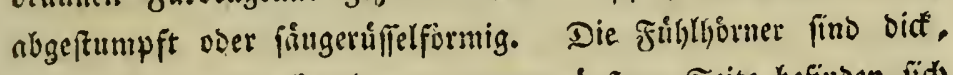
rund und pfriemenformig, an Deren supern Eeite befinsen fid) bie 2 rugen anf einer bejondern, erhobten uno an biefelbe ange:

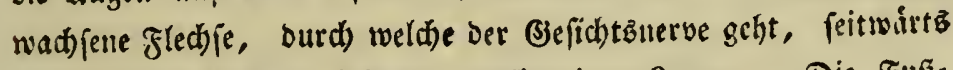
binter biejen befindet fid) ein ohyformiger Sappen. Die Fußs= fohle ift platt, vorne breit, abgetundet uno geft uad bintell etwas fid)måler zu.

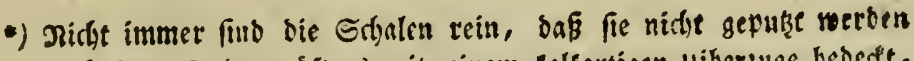

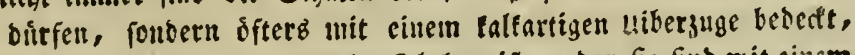

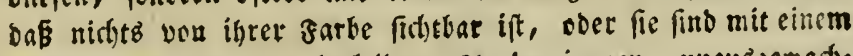
meopartigen uiberzuge befleibet. Xn ben jungen, unauвzerrart)=

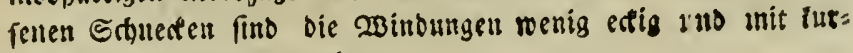
jen feifen Saaren bewartien. 


\section{$=88=$}

Sdyon åltere Raturforfder befonders. Sifter und Bicoffron haben bemerft, onß bey biejer Sd)nede bie Giefdled)ter getrenut

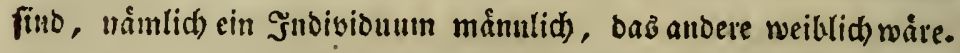

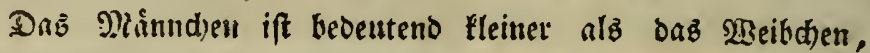

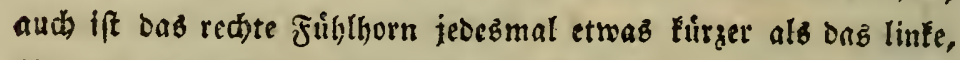

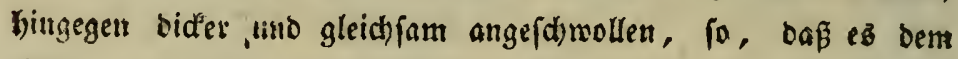
Sopfe melye als bem fíblhorn gleid)t. Man fonnte mit unges

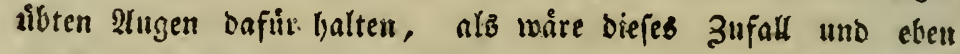

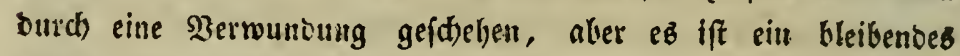
Merfmal, weldjez gifter aud) fobon angegeten hat.

Su biefe Ed)nerfe bie walkre Helix vivipara. Linn. ift,

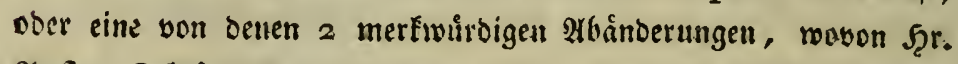

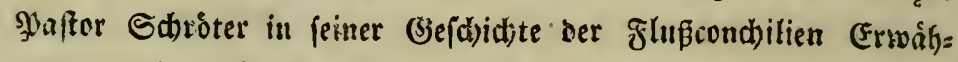
nung madyt, tiberlaffe id) anteren Paturforfd)ern zur (Ent/d)ei: bung. Man finbet fie in firlftetenden Maiffern, Gráben uno 2Beifern auf einem thonigten, (d)lammigten Boden. $B e y$ Marfts

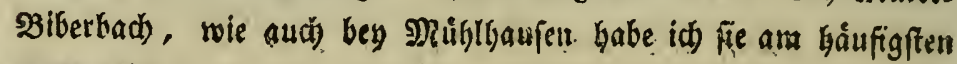
augetroffer. 


\section{Helix nemoralis. Linn.}

\section{Die Rieberryfortherte.}

H. testa imperforata fubrotundo laevi diaphana fasciata, apertura fubrotundo-lunata.

Linn. Syst. Nat. pag. 3647. Sp. ros.

Gualtieri Index Teft. Conch. Tab. I, Fig. R.

Edricters Ero: Eondjilien um \$ryangefferd, Tab. 2. Fig. 29. 30,

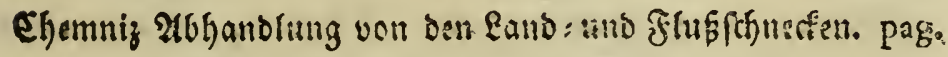
144. Tab. 132. Fig. 1196-1198.

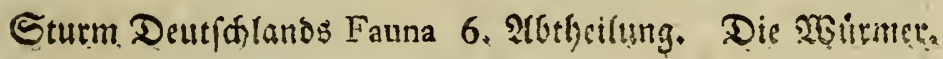
2. Szaft. Dns 6. Siupfer.

$\$$

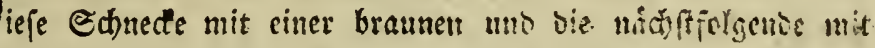

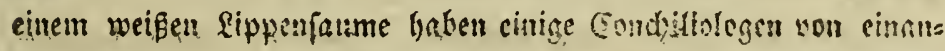

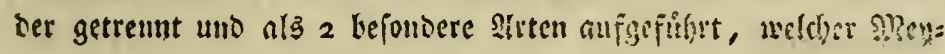
nung id, vorberfand nicht entgegen fent fann, bib ib mid) stl=

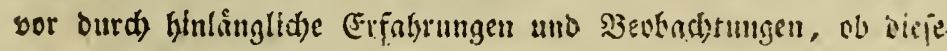
benden Sdnecten fid) nie z"jommen begatten warton, Bnson

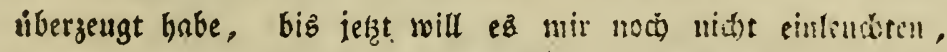

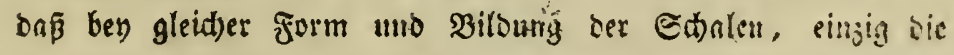

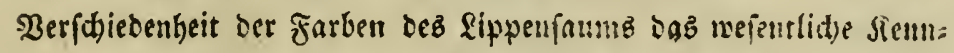
zeidyen eines Unterfdiebez bender arten fry founc.

Da biefe Ednecfe allgemein tofsum ift, fo sinube id,

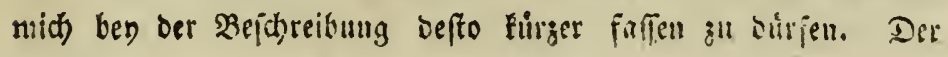




\section{$=90=$}

Durdimeffer ber Sdjale ift 10 Plarifer finien. Sie ift Ruget= formig, briun, aber bod) ni(d)t leid)t ferbred)lid), ourd)(d)ei=

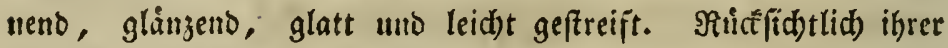
Farbe und Bånber giebt eż veridjicbene Epielarten, námlid): rethgelfe orne Bánber, weiplid)e uno gelbe nit 3 fdmalen uno breiten Bâubern, weld)e bey standyen zujammengefloffen uno in Der Mundoffinng am zwenten Gendinte rótblid)hraun finto. Eie bat 5 erhathene, gewolbte Bindungen, welde fidh in einen ftumpfen Birtel endigen.

Die Munobffnung ift långlid), Galkmsubformig, ber \&ił"=

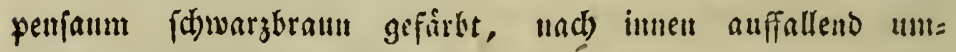
geidjlagen, Lilbet gleidjam eimen feiftenranto uno ift, wenn

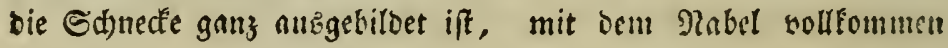
verwadjen. Das zhier ift $1 \frac{7}{2}$ zoll lang. Die obern gintsls

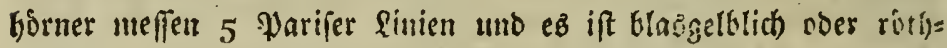

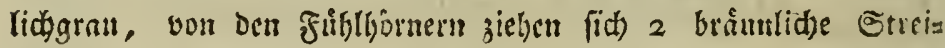

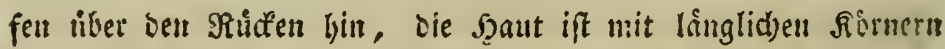
bejergt.

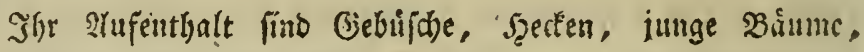
Bartenioande. 


\section{Helix hortensis. Muill.}

\section{Die Seartenfifnecte.}

H. testa imperforata globosa: labro albo. Linn. Syst Nat. pag. 3649. Sp. 109.

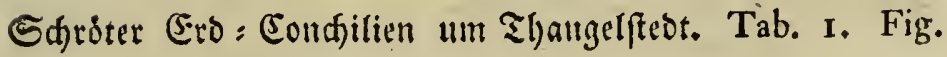
15. 16.17 .

Shenniz $\mathfrak{A}$ bhandhung von ben Eand : und Fluffidnecfen, pag. 146. Tab. 133. Fig. 1199. 120I,

Müller Hist. Verm. pag. 52. Nro. 247.

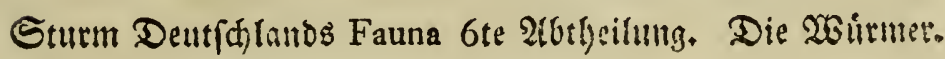
2. Sheft. Das 7 te Sillpier.

Diefe Sthnedte, bie in biefiger biegeno tibernlt hälfig gefun: ben wirb, varirt auferorbentlia), thid)t ollein it ihren Farken, fonbern aud) in ber 2 rnjagl ifrer Baincer. Man fintet folgente 3arietáten utto Alkánbertunget, alz:

a. zitronengelb ofne Bånber,

b. roth) ofue Bintor,

c. weiß̧lid) ofne Binder,

d. blazgelb mit cirem fomalen, fraunen Batise,

e. getb mit einem felgr breiten Bante,

f. gelb mit 3. 4. 5. formalen umb braumen 23íthern, weldye bey) manchen zujanmenflię̧en.

Shre Sd)ale miß̄t im Durdumeffer 9 biz 10 \&inien. Mit ber

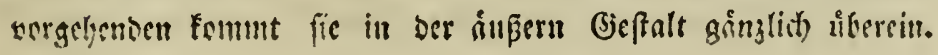




$$
=92=
$$

Sie ift 5mal gewunden. Die Munboffnung if ber vorigen

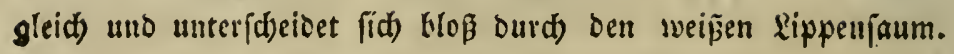
Dab ahier freft ber Helix Nemoralis affullid.

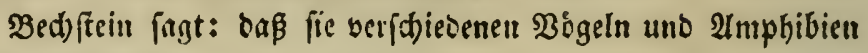
zแr Rabrumg bienen.

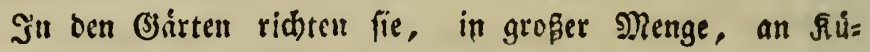
d)engenvid) fen viełen Sd)nden all.

Sie hat mit ber borizen einerley Infentbalt. 


\section{$=93=$ \\ Helix ftagnalis. Linn.}

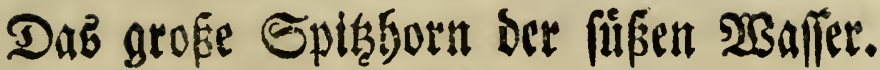

H. testa imperforata ovato - fubulata fub angulata, apertura ovata.

Linn. Syst. Nat. pag. 3657. Sp. 128.

Lister Hist. Animal. Angl. Tab. 2. Fig. 2I.

Gualtieri Index Test. Conch. Tab. 5. Fig. I. L.

Edwanmerdam Sibel ber Natur, Tab. 9. Fig. 4.

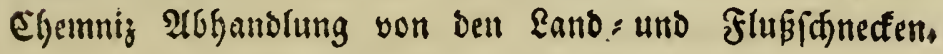
pag. I66. Tab. 153. Fig. 1237. 1238.

Sdjròters Bjefđidfte der Fluk: Condjilien, pag. 304. Tab. 7. Fig. I und 2.

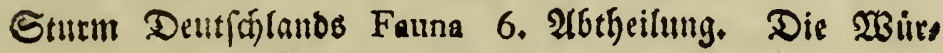
mer. Das 8te und gte Fitpfer.

II

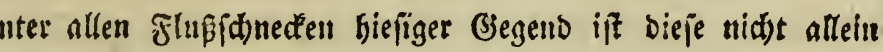
Die grospte, fondern aud) die gemeinfte, denn mall wirb nid)t leid)t einen (Braben, Zeid) ooer Sumpf antreffer, weldjer sou

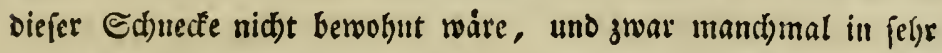
großer Renge. Die yor mir liegende Sdjuecte hat in ber Lánge einen Parifer 3oll und ro Sinien unb in ber gróften Breite beynahe cinen 3oll. Sie hat eine weişlid), blä̧braume, (d)mukgige

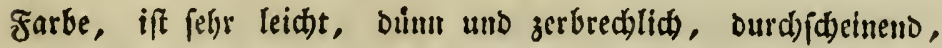
son auffen wenig, inwentig fegr glånzent, bie fánge berab 


\section{$=94=$}

fein geftreift, auf bem Mluden ber grofen Minsung ift fie mit

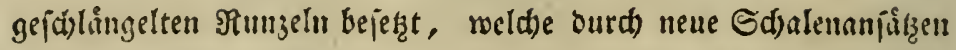

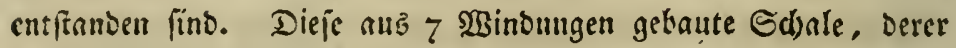

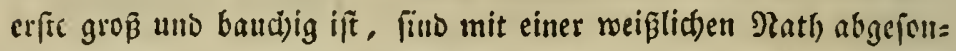
iert, bie ribrigen jetzen merllid) ab und endigen fid in eine Yfries menformige Spitze, weldhe braun gefiarbt ift. Die Mlunosffunng

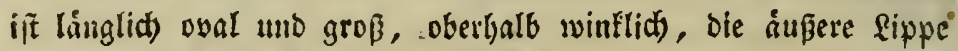
ungefíunt, fel)i bưnn uno gefdiweift, Die innere bilbet einen

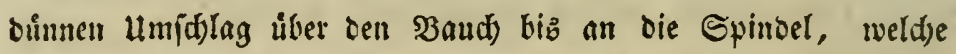
cine weiß̧lidgraue Farbe yat. Sie hat leitnen গtabel.

(5z ift bemerfenzwertl), baß man ourd) bie Epinbeliăule, weld)e fpiralförmig geidlångelt ift, bis auf bie Enofpizze mit= ten hindurd) feben fomm.

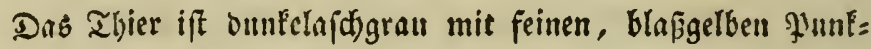

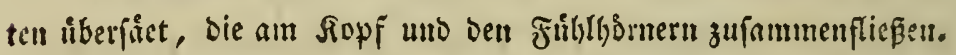

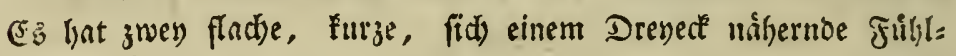

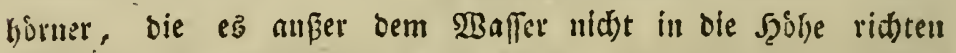

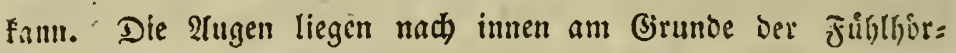

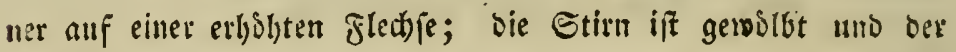
Sopf verlängert fid) sorne in 2 ansgefreitete Rappon, unter welden fid) in ber Mitte Der Mertiefung dic Muntopfinnung be= fittoct, weld)e in einem fleitun oreyectigen (bribd)en beftelyt. Die fuffoble ift langlin, dưn uno platt, mo vorne uno hin: ten abgerundet obme Đuufte. Die Suftrơjre liegt an Der Seite bor ånfern Mlunoungstippe, welde, wennn fie bie Dherflidhe

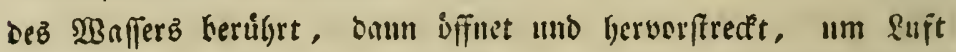

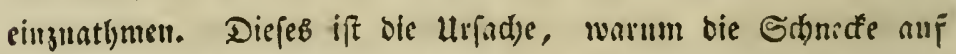

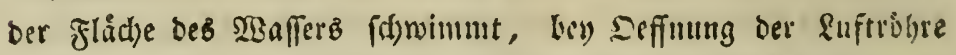
entffelt ourch bab Eintringen ber fuit eill Qaut, Milder bem 


$$
=95=
$$

Der Entweidung einer \&uftblafe auz bem 2 Baffer gleid) ift. Itns ter ben vielen leeven Sdalen bienen mande Den $\mathfrak{B a f f e r f p i n n e n , ~}$ weldbe bie Mlunoung mit ihrem Beppinft tibergiefen, zur beques men $\mathfrak{B}$ ofinung.

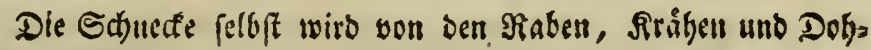
len gefreffer. 


\section{$=96=$ \\ Helix Putris. Linn. \\ Die Rafnifunecle.}

H. testa imperforata ovata obtusa flava: apertura ovata.

Linn. Syst. pag. 3659. Sp. 135.

Ginnani Opere Poftume Tab. I. paludosi Fig. 3. 4. beq Fig. 4. find bie fällillodner viel zu lang.

Gualtieri Index Test. Conchil. Tab. 5. Fig. H.

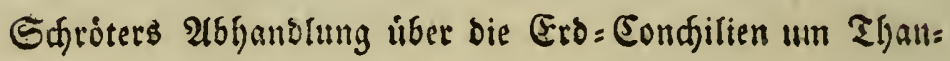
gelfedt. Fig. 2. 3.

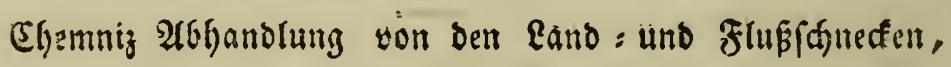
pag. 178. Tab. 135. Fig. 1248.

Sturm Deutfalands Fauna 6. A6theilung Der Wurmer, I. Szeft Das lef̧te Supfer.

Tie Eondillologen haben biefe Species and bie amplyibiest: f(t)recte genaunt, weil fie auf ber (Froe und im $\mathfrak{B a f f e r}$ jugleict) leben Bann; id) babe fie nut ein einjigeșnal anf einem Strauch jibenio anf bem $\mathfrak{B a f f e r}$ in einem (braben bey fed)haufen, fouft

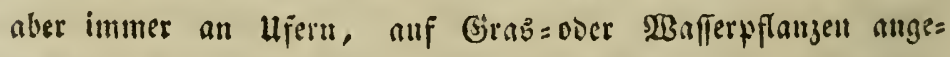

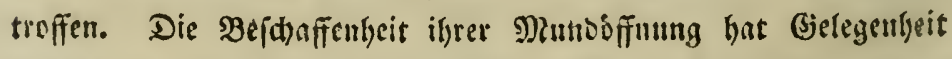
gegeben fie nit ocm 9) הumen Singnidumede zu belegen.

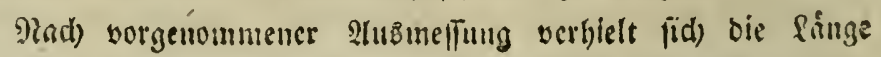

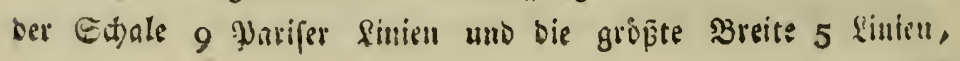




\section{$=97=$}

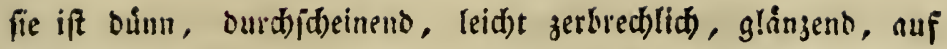
Der Deerflide laufen Dentlide Suerftreifen ber \&änge hernh,

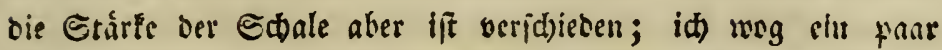
gleid)e vollig gerefnigte uno nasgewad)fene gegen einanocr, wo fid) ein Unteridtied von 4 Gran ergat. Sie bat gleichfan die

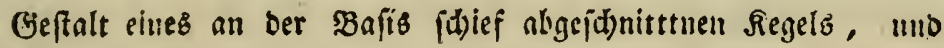

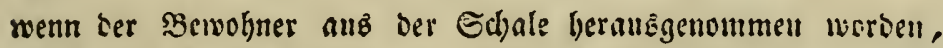

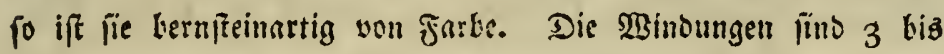

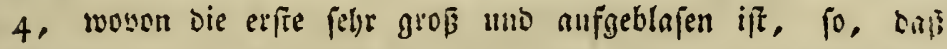
fie mebr als $\frac{3}{4}$ Theile ber Eonchilie cinnimmt, die lek̨tere $2 B i n=$

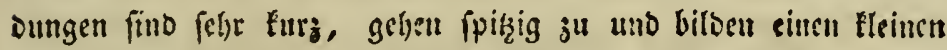
3opf. Dic s?undiffnug ift eyformig, ungefâumt, fegr groß und erweitert. Der Maindungšrand if to Dinme als bie Ed)nle felbft. Eie bat Eeinen Nabel. Das agjer felbft hat eine gell=

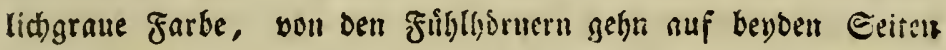
2 braute Strcifen ůber ben Plicteil hin, unt porn all bem Sopf if cit blaper brimulider flict fidthar.

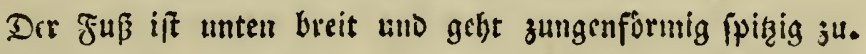

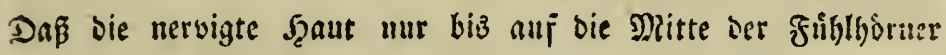

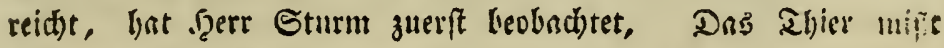

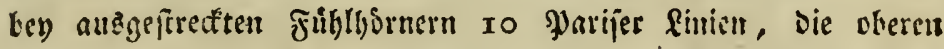

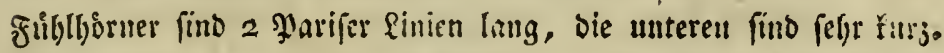

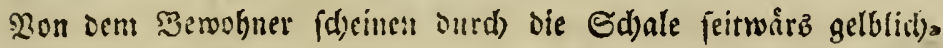

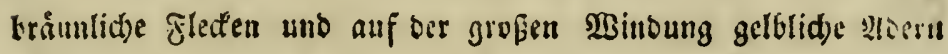
lebbaft burd).

Ëz gieft trod) eitte Barictît von meliber bie leere Echale

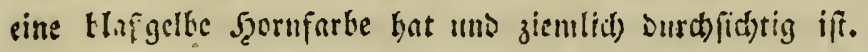

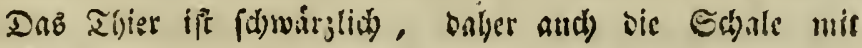
iemislben butretbram ift. 


\section{$=98=$}

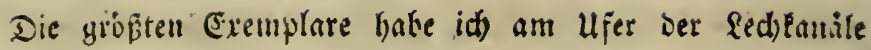
binter ben פlla añ $2 B a f f e r p f l a n z e n$ gefumben unb fins in

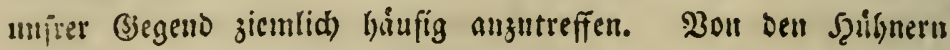
werben fie begierig scfieffen.

\section{Helix obscura. Mïll. Tab. 12. Fig. 2 I. Dạ Gerftenf́orn.}

H. testa conica fusca: apertura edentula; labro albo.

Linn. Syst. Nat. pag. 366r. Sp. I4I.

I.ister Hist. Animal. Angl. Tab. 2. Fig. 8. Jin biefem Werfe ift bicfe Edynecfe nur als Sontur ge: zeidnet uno jwar felgr unfemulith.

Argenville Conchiliolsz̧ie. Tab. 28. Fig. 15. Dicfe fris gur ift eine gan unridjtige afbbildoung und gebdirt nidjt biefger.

Müller Hist. Verm. pag. 103. Nro. 302.

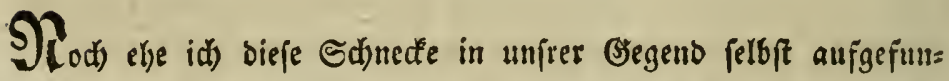
been batte, wurben mir melyere Exxemplare von meinem fdăkz=

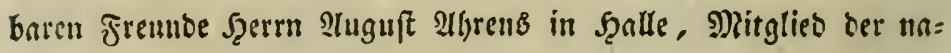

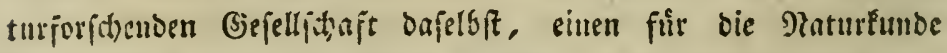

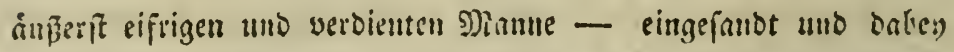

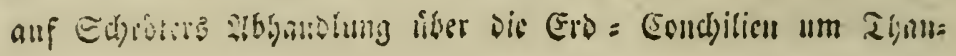




\section{$=99=$}

gelfted pag. 14r. Tab. r. Fig. 8. verwiejen, wofelbft fie unte: bem Plabmen Turbo dextrorsum versus, Die red)räge= orehte Erojobrable aufgefubrt wurbe; on aber bie Bejdyreikung to manig als Bas fintier mit biefer Ed)uede nibereinftimunt, fo

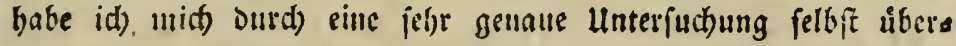
geugt, Eaßs biefeltbe feine andere alb bie yon Mitller (Histor. Vern..) bentlich befdrietene Helix obscura iff.

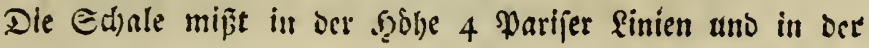
Breite $1 \frac{1}{2}$ \&inic. Sie hat eime gelblid)braure farbe, ift our (d)s fd)eiueno, wenig glinjent, bimujhalig, faft foraubenformig,

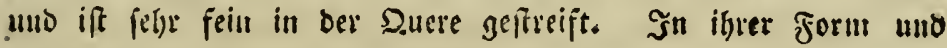
3ifoung but fie viel ribereinftimutetides mit Helix buccinata,

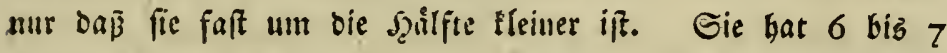
Biswinde, meldhe wenig baudjig fino, in eine fumpie Epitze auggeten unb burd) einen fleinen Einfobuitt akgefonbert finb.

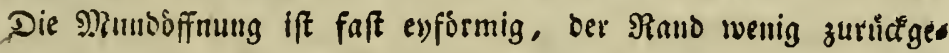
logen ober idwach) gefåunt. Die fiumale weiflid)e Mlinbungass lippe verurfacd)t burd) bas Inliegen an Der Spinbel eine lleine fobiefe uabelifigulide Epalte.

IBeun die Edale nod von bein lebenden Thiere berwolgnt wirb, fo bat fie eine braungelbe Farbe.

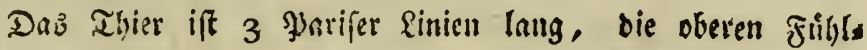

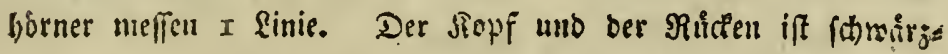

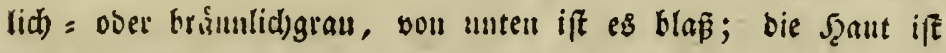

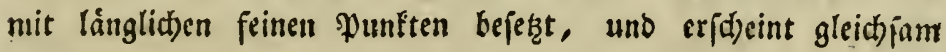
punftitt.

Diefe Edinnecte ift in unfrer biegent rat und felten, uns

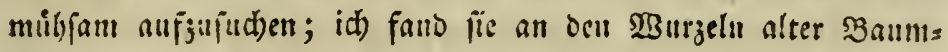

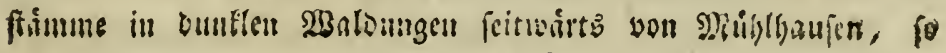




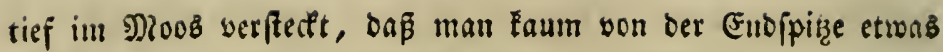
bemerter founte.

\section{Helix buccinata. Mihi. Tab. I2. Fig. 22. Die bautjige Staraubenfanecte.}

(5 Soe id Helix obscura in hiefiger Giegent anfgefunden hat:

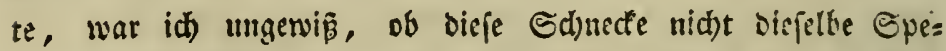
cieb mire, nadher liberzengte id) mid) aber ourdh gennate 2 ers

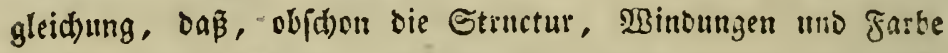

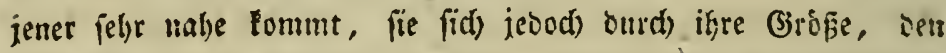

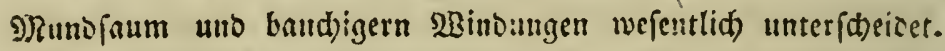
Da目 Helix obscura nid)t firi eine junge von Helix buccinata gehaltun werben foume, berweift, dấ fie solfig aus=

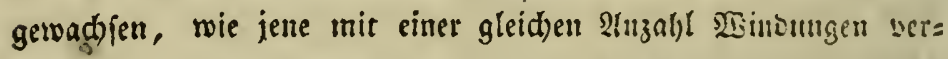
feben iff. Bon einigen (Eondjiliologen ift fie jebod) wagridjein= (id) mit Helix obscura firr einerley geffalten uno oansit vels wed)felt worben. Die hierben folgende Sefichreibung uno 2trkits bung wirb beutlid) zeigen, Daß̧ fie gewis eitue antere unt bie ift, weld)e ber uiberid)rift ent(prid)t.

Fgre \&ånge betright 7 Parifer finiell unb iu ber grofiftelt

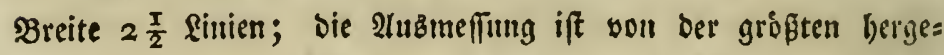
nommen, benu fic fommt aud sfterz etwab fleinet vor. Eie hat eine faft cylimberifdse form, bie nad) oben etwas (d)miler wiro, ift Durd)fid)tig, fettglinzeno, yon másiger Etirle. 2tuf

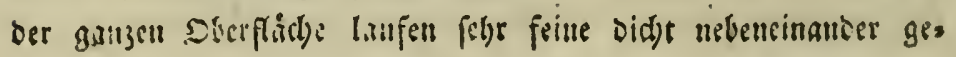




\section{$=\mathrm{IOI}=$}

Pekgte Streifen längft ben $\mathfrak{B}$ indungen hetab, und menn ber $\mathfrak{B} e$ c mohner aus ber Edjale herausgenommen wirb, fo hat fie eine bellfaftanientraune farbe. Mit dem Thier ift bab (jelhăufe

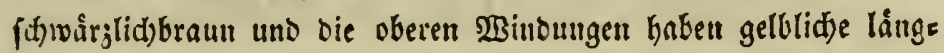
lid)e flecten unt Etreifen. Sie bat 7 red)tsgedrelfte etwas

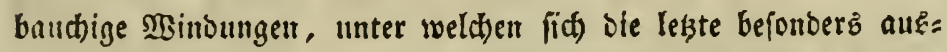
zcid)net; fie verengern fidh aber allmåglig unt enbigen fid) in siue abgeftumpfte Epifze. Die Runbsfinung if faft eyformig, ber umgebogene Sippenfaum hat eine glânzenbe róthlidgraue far: be unto bitbet binter fid) eigentlich feinen $\mathfrak{R a b e l , ~ f o n b e r n ~ n u r ~}$

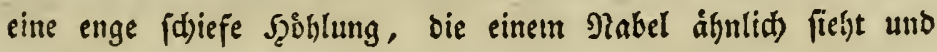
uni: biz an die Epindelfáule reidt.

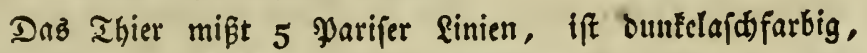
mit fdsmirglid)en Eleinen \$unften bezeid)net. Die fabenförmigen Füblliorner (novon bie oberen I sinie meften) uno ber Rưden baben eine fdiwirzlid) garbe. Die fdwarzen slugen fitzen wie bey allen (Erojönnecten (aurgenommen Carychium) auf

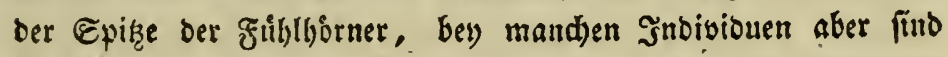

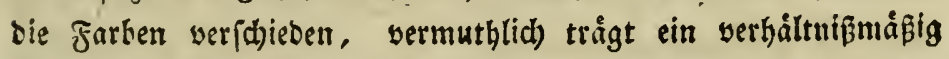
bohjerez Alter hierzu vielez bey, fo, baß pie in eine gleid)formige fdrwarlidgraue farbe úbergeben. Der Fuß gelt in eine abge: fumpfte Spize aub.

Sie geljort in unfrer Biegend unter bie Seltenfeiten, nran

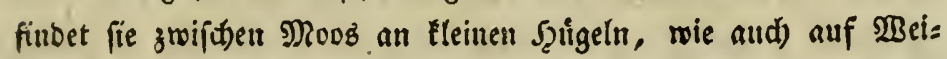
Denftráuden in ben \&anbbolzmaloungen in ber Begend von Berft: hojen, Mưblhaujen unb aud an ben fechtanálen binter bem

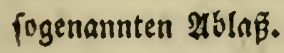




\section{$=102=$ \\ Helix lubrica. Martini.}

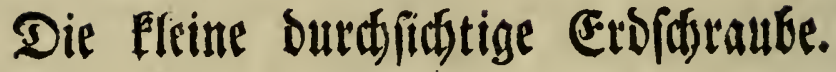

$H$, testa conica fulva nitida pellucida 2cuta: apertura edentula; Labro rubente. Linn. Syst, Nat. pag. 366r. Sp. 142.

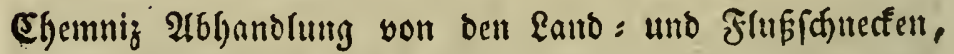
pag. 167. Tab. 135. Fig. 1235. Helix fub. cylindrica. Linn.

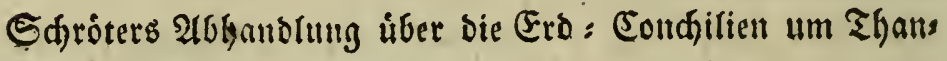
gelfert, pag. 141. Nro. 13, Fig. 9.

(5)

eoffroy hat biefe fleine nieblide (Erbidnecte Turbo nitidus

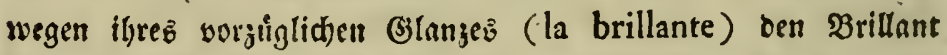

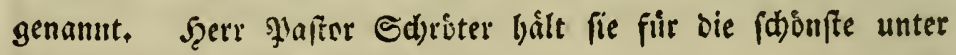
ben Eleinen Sd)raubenidureden.

Die Sd)ale hat in ber fånge hódfftenc 3 Ylarifer finien unb in ber $\mathfrak{B r e i t e}$ heynahe $I \frac{T}{2}$ finte. Sie hat eine fegelfor= mige Biefalt, ift vollfommen burd)fid)tig, felsr feinfd)alig, leid)t jerbred)lid, burdinans glatt ohne Etreifen, uno bat cinen Spies gelghan. Die farbe ift fdoun hellbraun. Eic hat 6 fdrall= benformige Winturngen, weldhe Durd) cintell feinen Ereifent ab: getbeilt fint uno endigen fid) besm $\mathfrak{B}$ Birbel in eine ftumpfe Epize. Shre Thunbsfinung ift balbhergformig, mit einer roth=

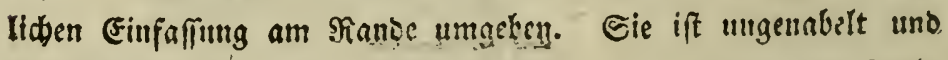

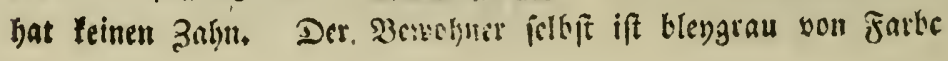




\section{$=103=$}

unb hat in ber sange 2 Y̧arifer Sinien, bie oberen filflforner meffen $\frac{T}{2}$ sinie.

Ran findet fie an Ufert, auf feudtem Micjengruts

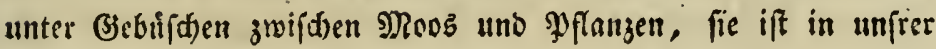
(B)egend felyr auşgebreitet.

\section{Helix tentaculata. Linn.

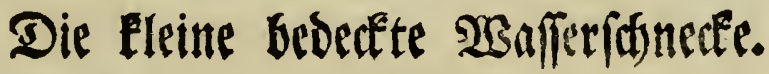

H. testa imperforata ovata obtusa impura, apertura fubovata.

Linn. Syst. Nat. pag. 3662 . Sp. 146.

Gualtieri Index Test. Conch. Tab. 5. Fig. B B.

Sd)lotterbecf Acta Helvetica. Vol. 5. pag. 281, Tab. 3. A. Fig. 19, 20, 21 .

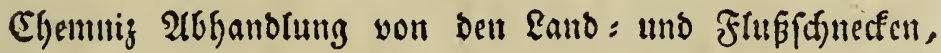
pag. I75. Tab. I 35. Fig. I245.

Sthroters (jefdid)te ber. Flup: Condjilien, pag. $32 \mathrm{I}$. Tab. 7. Fig. $19-22$.

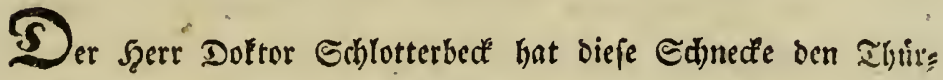
buter genannt, weil fie fich) bey ocr geringften Berrilgrung gleid)

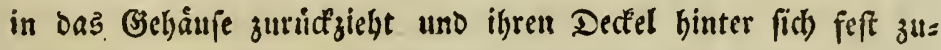
fdlieşt. Die Ránge Der Sdale hat 5 parifer Sinien uno sie Breite ber gropen WBinbung unipt 3 finien; fie ift felys glatt, 


\section{$=104=$}

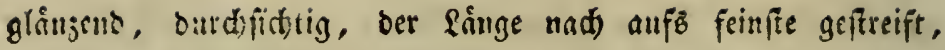
sout måpiger Etirute, hat eine gleidjaum fegelförmige Bieftalt uno eine einfärbige gelblid)e Şornfarbe. SBBent aber bab Thier nod in ser E(j)ale lelt, to ifit fie sunfelbram, mit gelbliden fles ctent melgr oder weniger bebed, weld)es aber nur von gereinigten Exemplaren fann geforgt wertoen; Denn fo wie fie von manchen Drten aus ben Baffer Fommt, fo ift fie ofterz ganls unit einer falfartigen Sinve úbergngen. Sie hat 5 in bie Scobje gezogene Minisungen, weldye fein zugefpitht anzgehen uns woson bie erfte

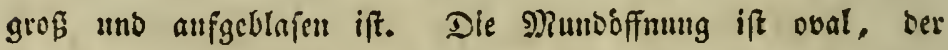
Rand glatt uno nid)t umgeld)lagen. Sie hat keinen Nakel. Der Deffel, momit sas ahier fein Biehåufe genan verfhlię̧t, if meiglid), bonfurbig, mit ringformigen Sinien ourdzogen unb im Rittelpallikte um wenig vertieft.

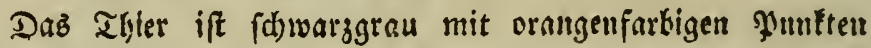
getiopfelt, ber Sopf ift långlid)runo uno abgeftumpft, an ben 2 fabenformigen fpistgen friblhorttern befinben fich an ber aufs fern Seite die Ingen; bie Funjoble ift vorme kreit, wenig ectigt unb gel)t und binten etważ (a)máler zu, an welder ber Dectel allf ber otern Seite befeftiget ift. Die fänge bes Thierz, fo wie ez an ber Geite im Blafe berauffriecht, Ketrịgt 4 Pari= fer sinter.

Diefe artige Speciez findet man biuftig in den meiften

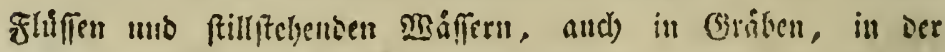
gunzen untiegenten Begento von Irugäburg. 


\section{$=105=$}

Helix auricularia. Linn.

\section{Die Shrithnerfe.}

H. testa imperforata ovata obtusa, fpira acuta brevissima apertura ampliata.

Linn. Syst. Nat. pag. 3662. Sp. I47.

Gualtieri Index Test. Conch. Tab. 5. Fig. G.

હallotterbeff Acta Helvetica. Vol. 5. Tab. 3. Fig. 27. 28.

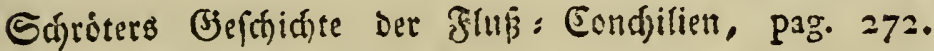
Tab. 6. Fig. $3-6$.

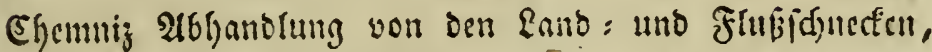
pag. 171. Tab. 135. Fig. I24I unb I242.

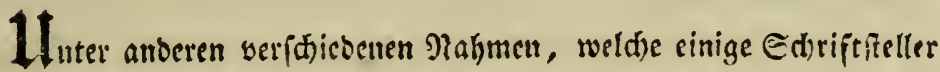

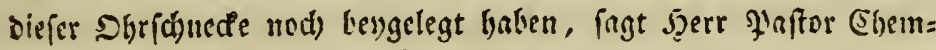

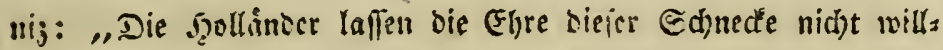
"falfren, cinem menfdlidfen Dhre zu gleidjen, fondern gethen "ilfr ben gialymen oç Måujeclerb."

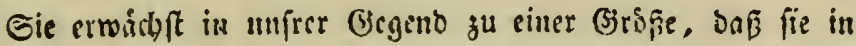

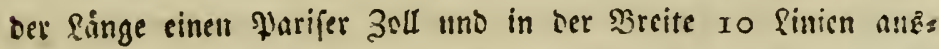

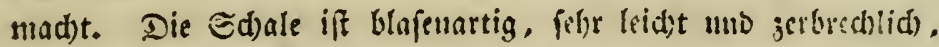

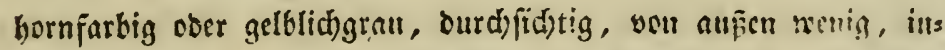
wentig fehr glinzento, faft glatt umo mit lánglidion Strcipen

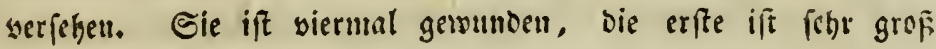
umb aufgeblafen, uno nimmt faft bent ganizen umfang oer Edha: Ie ein; Die librigen finto folfe furr und entigen fich) in eine fdist: 


\section{$=106=$}

fe Epize. Die Munbsffnung ift ungsfiumt, auferorbeutlid)

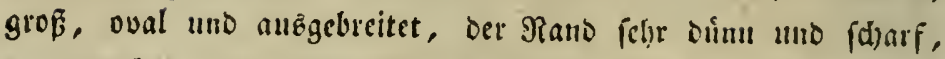
an mand)en Subividuen etwas cingebogen, von ankent an ber

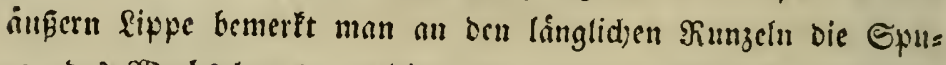

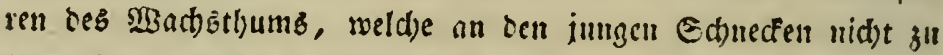

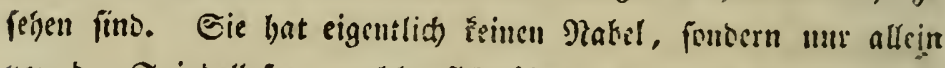
von ber Epindellefze, weld)e fid) rifer den $\mathfrak{B a u d}$ nulegt, ent= ferfyt melye noer weniger eine enge Berticfung ober nabelífylidge Epalte. Dic Epinbelfäule ift meiglid), etwaz gersunben noor eingebogen uno fieht eirem menfdlid)en Dhre nid)t unithulis.

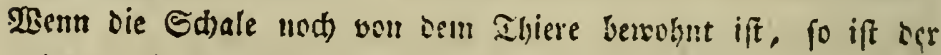

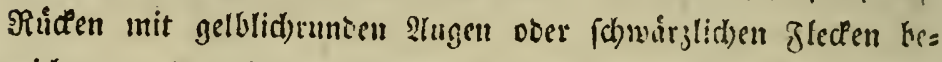
seid)met. Daz Thicr ift graulid), mit weiflid)en P'unften le=

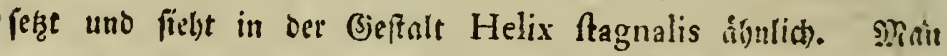

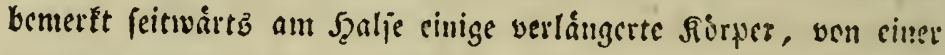
wurmformigen (Beftalt, meld)s um zu cutfibcioen, ob fie ism

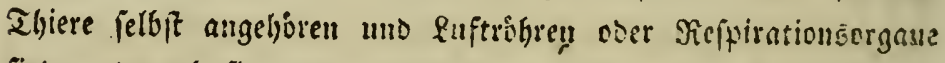
finb., ober sb fie auserwefentlid), wirflich 2Burmer, aljo alz allfillig betrad)tet werten miffen, id) sabjin geftellt fenn laffe; on genanere ployfiologifde unterfird)ungen ber ziwed biefer 2l: beit nid)t foun foumtct.

Nan finbet nod) eine atbait biefer Epecies, welde fids ourd) einen fdmalen melye gefrecten $\mathfrak{B a n}$ uno eine enformige linglid)e Runboffmung yon biffer unterf(b)eibet. Eie ift abgez

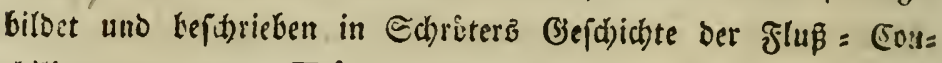
dillien pag. 3r8. Tab. 7. Fig. I2.

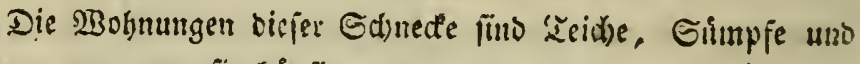
Griben, wo man fie bioufig anjutreffen plegt. 


\section{$=107=$}

\section{Helix Carychium. Müll. Tab. I3. Fig. 23.}

\section{Die Enttenfínccis.}

H. testa hyalina fubconica glabra: apertura ovata dentibus intus fesquitribus munita. Linn. Syst. Nat. pag. 3665 . Sp. 156.

Müller Hist. Verm. pag. 125. Nro. 321. Carychium minimum.

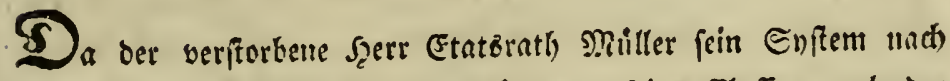
ber allgemeinen $\mathfrak{B}: t$ toung ser $\mathfrak{B}$ sirmer, feine Etaffer nad) Der

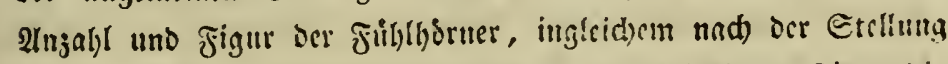
ber 2lugen feine Gejdledter, nad) oer afwedjélnoen Figur ier Edalen aber bic uluterabtheilungen ober battungen gethiteet bat, fo if̂́ ă

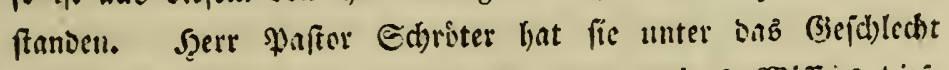

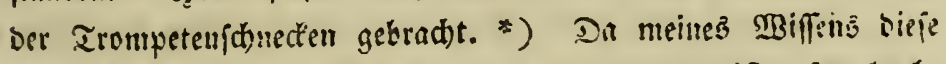

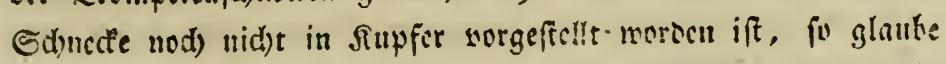
id), Da unangenelym jemn wire.

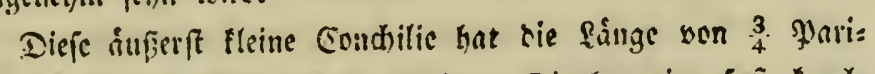

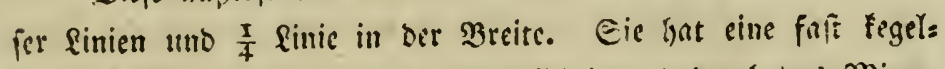

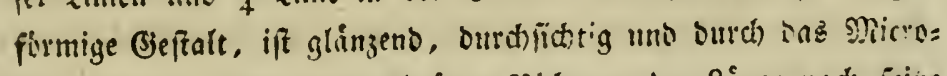

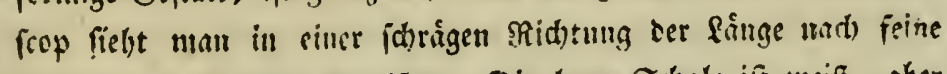

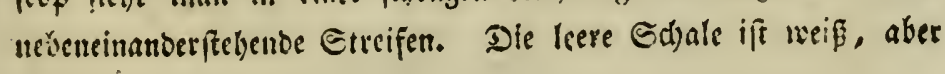

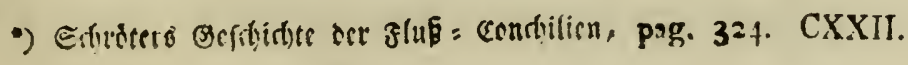




\section{$=108=$}

menn bas lefende Thier nod) barinn befindlid) iff, find bie 3 oberen umginge gelblid) gefoirtb. Sie bat 5 gewollbte 2 Bintoun= gen, bie burd) feine (einfchitte von einander akgefondert fino sub in cine finnte Enofpiege ausgeken. Die Munboffung ift cyfurmig, etwab geornicft und mit 2 anperorbentlid) fleinen Billund) ver vergen, wovon einer nuten an ber Epindelfáde, ein sweyter am \$antbe ber Epitublfäule befindlid) ift; bey mand)en Sindibibuen jeigt fid) and) an Seitenranoe in ber Mitte eine Fleine Errfólumg ober eine Epur eines folchen 3åand)ene. Der Mhindungärand ift etwab zuriocfgetiogen uno die $\mathfrak{B a f i z}$ ift unge=

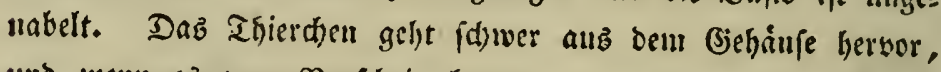

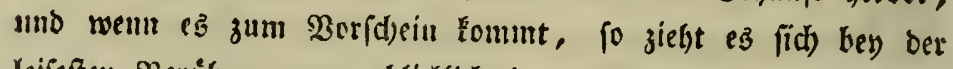

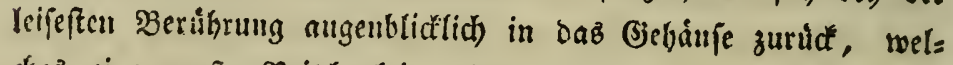
d)ez eine grop̃e Reikgarf́cit zeigt. (Es hat eine weiße ourd)=

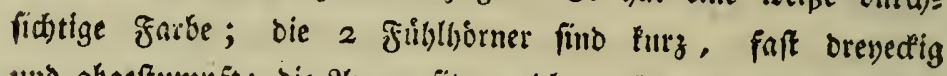

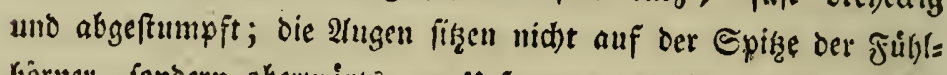

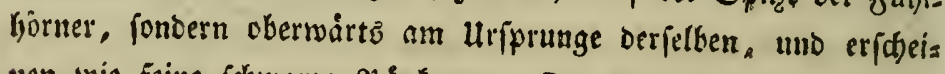
nen wie feine fdmarze Yuintgen. Der Juß iff felyr furz.

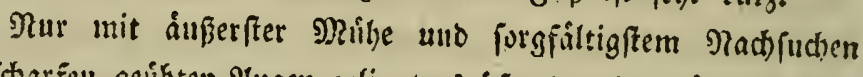

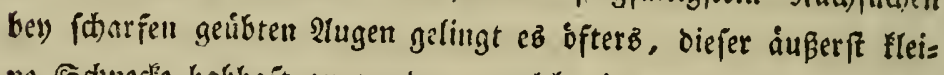
ne Sd)нedle habhajt zu werben, welde in unfrer (Jiegento unter bie 2 Injagl ber allerkleinften zu zablen ift. Эa) babe fie binter

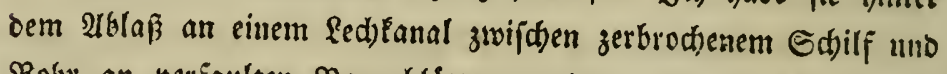

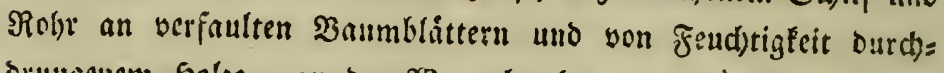

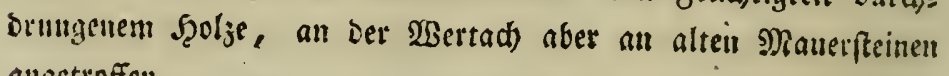
angetroffen. 


\section{$=109=$}

\section{Helix Corvus. Schröter.}

\section{Det Pinbe.}

H. testa imperforata ovata nigra: apertura ovata. ( $\mathrm{H}$. palustr. fimil.)

Linn. Syst. Nat. pag. 3665. Sp. 203.

Lister Synopsis Tab. 124. Fig. 24. Müller Hist. Verm. pag. 131, Nro. 326. Bucc. palustre. Sd)róters Beffdidfte ber Fłnß : Condjilien, pag. 307. Tab. 7. Fig. 3. 4.

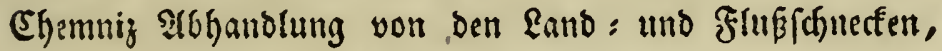
pag. 170. Tab. 135. Fig. I239 und 1240.

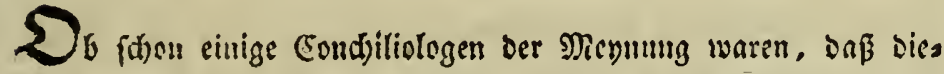
fe Ed)necie cine Barietat you Helix ftagnalis feye, aud)

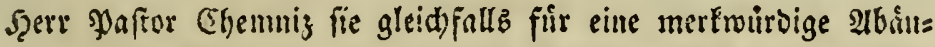
terung berjellien gehalten lyat und zroar :me unr, wie mir'z fdeint, feincm Branofakze, "bie Battungen fo wenig als mog=

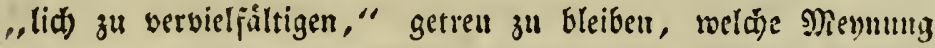
er damit redtfertigte, Dấ bey bemienigen Exemplare, welthes er

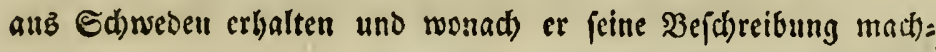
te, bey) einem loobern uno reifern Ilter biefes Exemplar vcr:

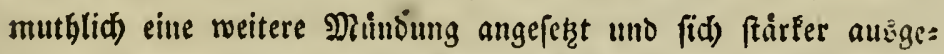

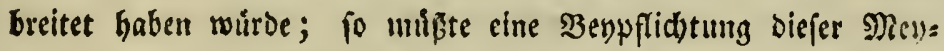
nung won meiner Eeite nudb) forgfiultigfter $\mathfrak{B}$ ergleitbung mrine: aubgrwad)fenen (Exmptare jesod) gatij gegen meine eigne Litre: 


\section{$=110=$}

scugung fireiten, wie mir (d)on aud) jeber, ber nur bie bier fols genoe uno meine Befdrribung von Helix ftagnalis aufmert: fan gelejen hat, gern beyptimmen wird: Daß bie Uuterihei= Dunggimerfmale zu wefentlich fino, um bisfe Sdunecte nicht firs eine bejondere Gattung balten zu muifen.

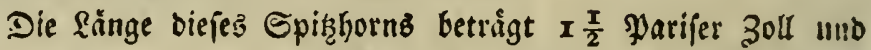
ill Der Breite, wo fie an biffifell ift, $\frac{T}{2} 30$ ll ; fie fommt abet

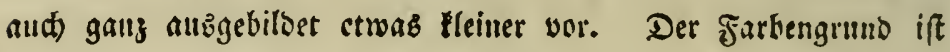
falwarbbraun, mitunter inb blåulide fpieleno uno hat bin uno

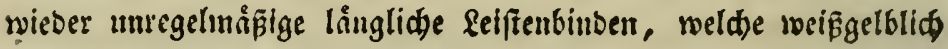
und braunroth fins. Sie ift thurmförmig gebant, bic \&ånge berab mit Did)ten Duerftreifen bezeid)net, wenig glåmjents, jient=

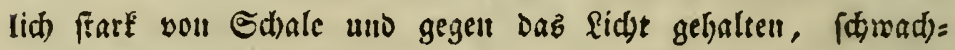

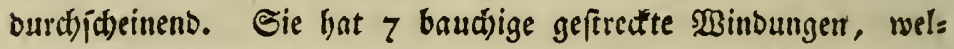
d)e Durd) Hleine Bertiefungen getrenut fint und in eine foine Epizze enben. Die Munboffinung ift lainglid, enformig, ins wendig sioletbraun; Der Mlindungärand felge bủnn und fdarf,

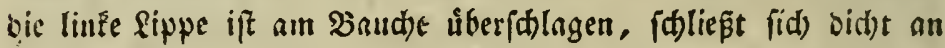
Die Spindel an und hat cine rothlidgraue Farbe. Sie hat fei=

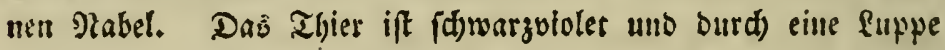
betrad)tet mit feiner blägetben \$unten bezeidinet, uno in bes Biloung sem gropen Spitzhorn ảbnlid), Daber glaube id) Die 3ej(f)ecibung hier übergelhen zu Pỏnnen.

Man finbet dieje Sdfuecte in Gefellịjaft mit ber Helis Atagnalis. 
Nerita valvata. Müller. Tab. I3. Fig. 24. Daz genabelte Pofthorn, beffen Ibier eis nen Federburiti trágt.

N. testa planiuscula: apertura circinata. Linn. Syst. Nat. pag. 3675, Sp. 22.

Müller Hist. Verm. Pars II. pag. 198. Nro. 384. Valvata cristata.

Sdrane Sammlung naturbiftorifaner uno phyfllaliface Aufiüle, pag. 321. Tab. 5. Fig. 9-13.

Sdroters (jefdidate ber Fluß : Condilien, ’pag. 240. Tab. 5. Fig. 26. A. B.

(3)melin แm es úberfehen baben, biefe Sduede aus Edroters flup: Sondjilien zu cititen.

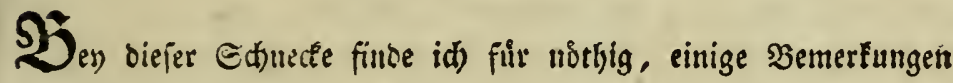

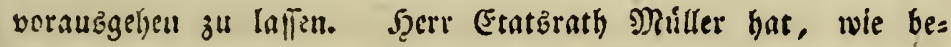

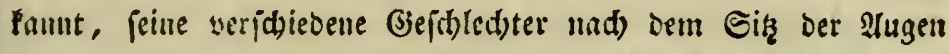
gevibnet, mithin ift bey ihm biefe Sdonecte bie einjige Gattumg uno zmai aub bemr Grunde Oculis ad basin postice; Da et aber beym Feberbufdrtáger, Nerita piscinalis. Müller, (Helix fascicularis. Geoffroy.) mur bie leere Shale gefunben und bab Thier felbft uie gefergen hat, fo lonute er folglid bieje nid)t alz sille zweyte (Battung auffübren, weil ber Sikg ber Ingen bey) Der eiten gernde alb wie bey ber andern ift, naims lid) oben an (osumbe ber beysen fillighormerm. 


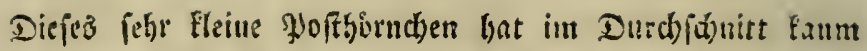
I $\frac{T}{2}$ lyarifer Rinien uns ift bemuad wohl die fleinfte unter ben Mofthornidinerfeir. Die Sdale ift oben uno unten platt, buduls lid)gelb oder hornfarbig, burdfid)einenb, wenig glånzeno, feills rhalig und felyt fein geftreift. Sie befteht aus 4 SBinoungen, Dic runo gerwolbt, um ben Mittelpunft gewunben uno ourd) ci=

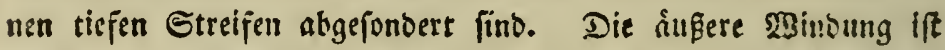

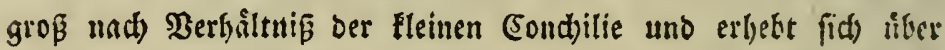

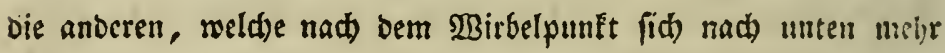

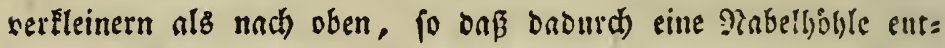
ftel)t. Die Munboffnung ift vollig freistano uno legt fid) bid)t

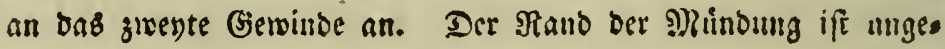
fâumt uns fo-bưn als bie Echale jetblt.

Das Zgierd)et hat eine weiflid)graue Farbe. Der Ropf bildet faft einen Eylinter, weld)er surd) eine leict)te Ritugêfurd)e

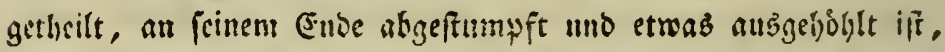

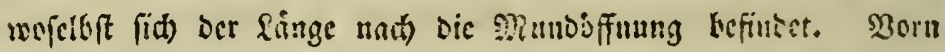

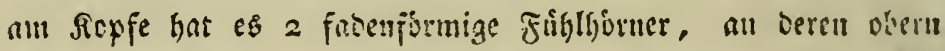

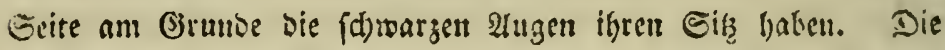

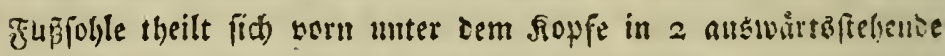
Sappen uno geht uad) hinten abgerumbet breiter ju, an weld)en

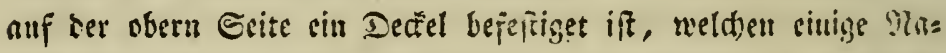

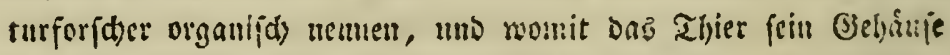

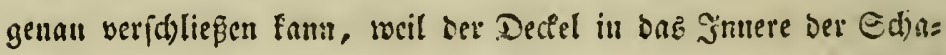

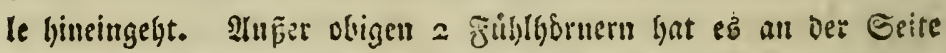

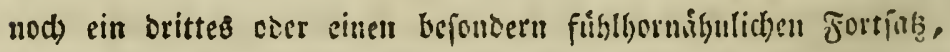

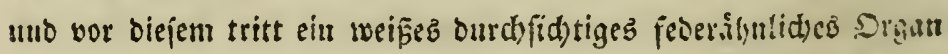

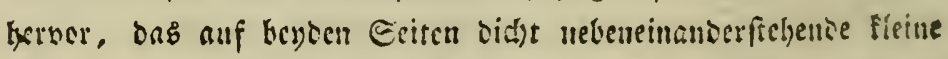
Esitsufiperdyen but, wild)e man fîr die firemen bialt. 


\section{$=113=$}

Unter ben vielen biejer Sdinecfen bie id beobadtete, muse be id) nur bey einigen ben Jeoerbufd beutlid) anfid)tig; bas Thier frectit ifn im fitiechen nid)t allemal bervor uno aud) nidise fo weit uno fo sillig alz ber Feberbu[d)tráger heraus.

Cie lebt vorzliglid) in Sámpfer, auf $\mathfrak{B a f f e r p f f a n z e n ~ u n o ~}$ Sd)lamm; bey Dherfaulen, Sangmeit uno bey Steţling ift fie anzutreffer. 


\section{$=114=$}

\section{Patella lacustris. Linn. Tab. I4. Fig. 25.}

\section{Die Fluppatelle.}

P. testa integerrimma ovali membranacea: vertice mucronato reflexo.

Linn. Syst. Nat. pag. 37 ro. Sp. 97.

Argenville Conch. Tab. 27. Fig. I. Theint mir eljer $\mathrm{P}_{2-}$ tella fluviatilis ju fenn.

Argenville Zoomorphose, Tab. 8. Fig. I. Diefe Zెeidfs nung ift von Der Flluppatelle ganz verffieden.

Ginani Opere Post. II. pag. 50. Tab. 2, Fig. Ir. Illy fentutlich uno Eumftos geftoden.

Sortinifajes Migajin 4. Sand, pag. 230. Tab. 7. Fig.

2. 3. (Ein Piadjptid, welder and) Diefe Yatefle nid)t eigentlich vorffellt.

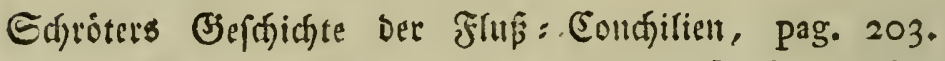
oTab. 5. Fig I. 2. 3. Diefe Zeidfnumg hat

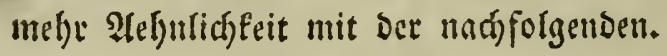

Jam Eimeiffen Syftem fehlt biepes Sitat.

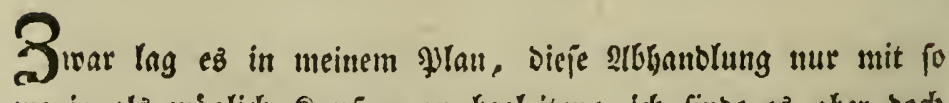

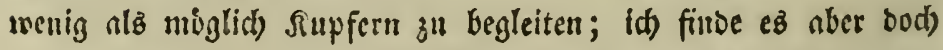
fuir nothwentig, foldte nod) mit einer łlatte zu bermefreen, nno zwar ans Den (Jirumbe, weil id) Diefe umb folgende gyatille in

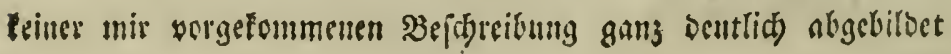




\section{$=115=$}

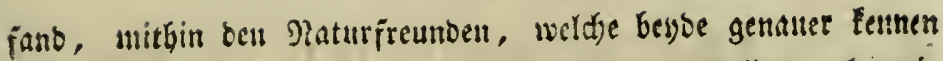
sund ridtiger your einander unteríheiden lernen wollen, als wle

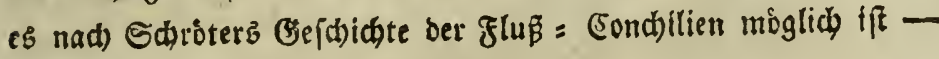
baburd) einigen Dienft zut erweijen.

Die Schale Gat im Durdmefier 2 parifer finien in ber

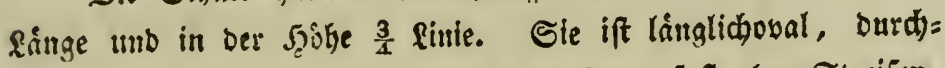
fïhtig, fobr buinn uno zart, etwab bieglam, faft ohne Streifen, bod) bemerft man feime 2uerfurchen, weldbe burd ble nenen

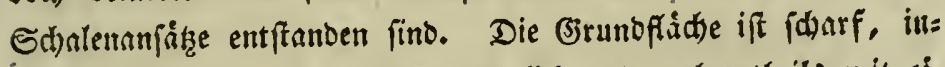
wenbig glärzeno, von außen meiß̧lid) unb mehrentheils mit ei=

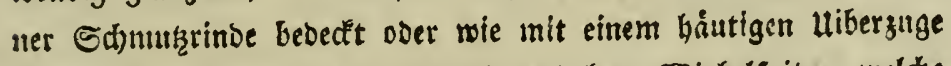

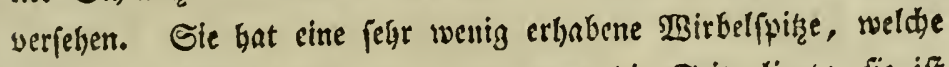

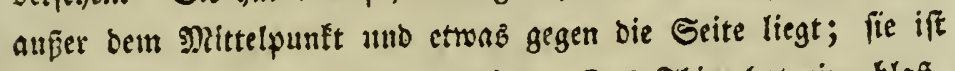

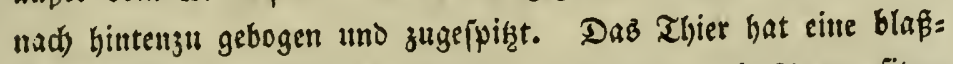
graue Farbe, ber fopf ift breit unb zugerumbet, die 2 tugen fithen unter an ber itmern Seite ber genthrborner, welde wegen ihrer Sd)wirge leidjt bemerft werben fonten. Der gup if länglich utto geht in eime abgeftuntyfte Spitze aus. WBenn man bie Eda=

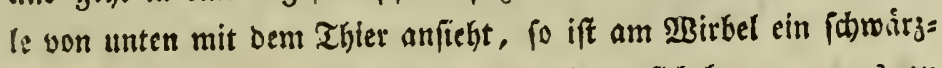

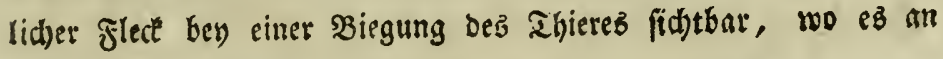
ber Sdale angefeftet ift.

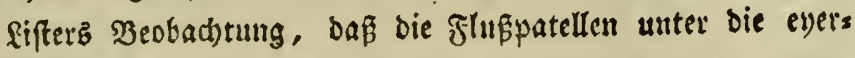
legettben Sdintthicte gethoren, ifr mod) nidht ganz erwizfen.

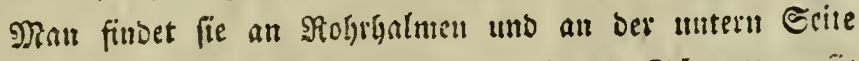

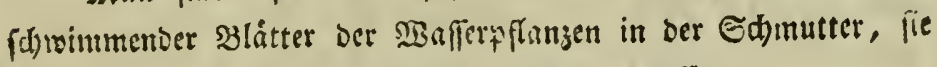
finb aber in unjer Biegend mat felten anzutreffen. 


\section{$=116=$}

\section{Patella fluviatilis. Müll. Tab.14. Fig. 26.}

\section{Die Dragoner $=$ Mathę.}

P. testa integerrima ovali fubcornea: verticis mucrone marginali, apertura ovali. Linn. Syst. Nat. pag. 37 I. Sp. 98.

Müller Hist. Verm. pag. 201. Nro. 386. Ancylus Aluviatilis.

Lister Hist. Animal. Angl. Tab. 2. Fig. 32. Argenville Conch. Tab. 27, Fig. I.

Gualtieri Test. Conch. Tab. 4. Fig. A A. und B B. Diefe $\mathfrak{A b s i f o u n g e n ~ f i n d ~ z w a r ~ g u t ~ g e f t o d b e r , ~ a b e r ~}$ in Der ßaunart mid Brópe you unfrer Patelle gang verfajicden, uno es burfte vielleicht eine an: Dere Specteo renn.

Sardtets. Siefdidate ber Fluf : Eondjilien, pag. 205. Tab. 5. Fig. 4. a. b.

Die Schale bat im Durdmeffer 2 Parifer finien uno in ber Scobje I $\frac{T}{2}$ Sinic. Dor Umfang igre: Girundfládbe bilbet ein

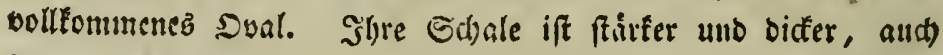

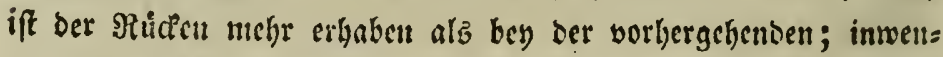
big ift bie Shiblung glinjent uno bat eine violetblänlide garbe, won ausen ift fie fdnmutiggrau mit einem bráunliden Epiberm uberzogea und som 2 Sirbel berab mit feht feinen lingliden 


\section{$=117=$}

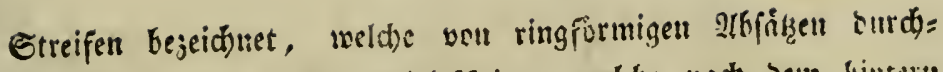
(d)nitten werbell. Die saithelffiçe, meldhe nad) bein hintern

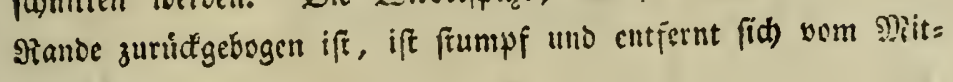
te?punfte.

Dab Thier ift graulich, Der Sapp ift breit und gugerrittoet, und hat am Enbe eine fleine 2 ertiefung, unter welder fid) bie Munboffunung befincet. Die Slugen befinien fid) unten an ber inuern Eeite ber abgeftumpften Friblhiorner. Der guf ift liug=

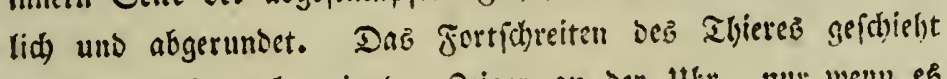

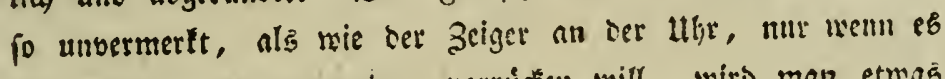
yon einem Srte zum antern vorrúcten wifl, wirb man etmas

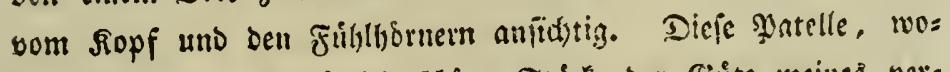

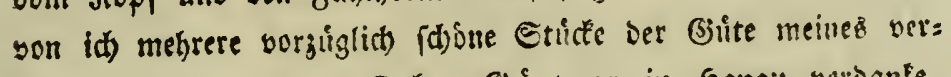

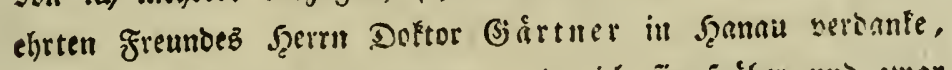

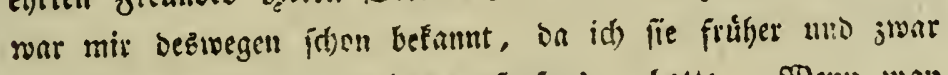
nur einige wenige Exemplare anfgefunden hatte. SBem man siefe \$atelle mit Patella pectinata vergleid)t, fo findet man bin=

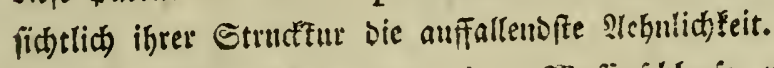

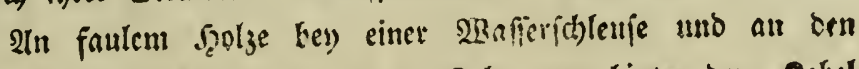

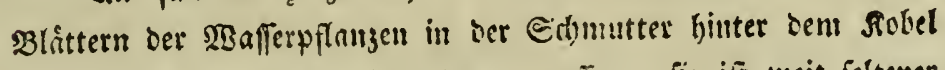
hake id) fie in hiefiger (jegeno angetreffell; fie ifit meit feltener

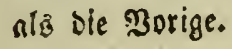




\section{Serzeitiniß}

Ser it biefer Mbbandlung vorfommenden Mufdeln, (Frb = und FIU = Eondillien.

(Die Sterndten weifen auf die Rimneidjen abtheilungen hin.)

Mya.

Mya pictorum. Linn. . . . . . . Ecite I

\section{Tellina.}

*** fuborbiculatx.

Tellina cornea. Linn. . . . . . . . . 3

Tellina tuberculata. Mihi. . . . . . . . 4

$$
\text { Mytilus. }
$$

*:* ventricosiusculi.

Mytilus Cygneus. Linn. . . . . . . . 6 Mytilus anatinus. Linn. . . . . . . . .

\section{Bulla.}

Columella obliqua, laevis.

Bulla fontinalis. Linn. . . . . . . . . 9 Bulla hypnorum. Linn. . . . . . . . . II

\section{Turbo.}

***** Turriti proprie dicti.

Turbo bidens. Linn. . . . . . . . . . I3

Turbo perversus. Linn. . . . . . . . . . 16

Turbo conversus. Mihi. . . . . . '. . I8 


\section{$=$ II $9=$}

Turbo quadridens. Geoffroy. . . . . Seite Ig Turbo tridens. Müller. : • . . . . . . 2 I Turbo muscorum. Linn. . . . . . • • 23

Helix.

* Carinatae, anfractuum margine acuto.

Helix lapicida. Linn. . . . • • • • 25

Helix incarnata. Schröter. . . . . . . . 27

Helix planorbis. Linn. . . . . . . . . 29

Helix complanata. Linn. . . . . . . . $3 \mathbf{E}$

Helix vortex. Linn. . . . . . . . 34

Helix lenticularis. Mihi. . . . . . . . 35

Helix Isognomostomos. Hermann. • . . . 38

*** Rotundatae anfractibus et umbilicatx.

Helix contorta. Linn. . . . . . . . 40

Helix alba. Linn. . . . . . . . . . 42

Helix hispida. Linn. . . . . . . . . 44

Helix pilosa. Mihi. . . . . . . . 46

Helix pomatia. Linn. . . . . . . . . 48

Helix arbustorum. Linn. . . . . . . . 5 I

Helix nitidula. Mihi. . . . . . . . . 53

Helix ericetorum. Lister. . . . . . . 54

Helix thymorum. Mihi. . . . . . . $5^{6}$

Helix nitens. Argenville. . . . . . . 58

Helix costata. Müll. . . . . . . . . 60

Helix rotundata. Argenville. . . ... . . 62

Helix obvoluta. Müll. . . . . . . 64

Helix crystallina. Müll. . . . . . . 66

Helix fruticum. Müll. . . . . . . . 6 ?

Helix fylvestris. Mihi. . . . . . 69 
Helix aculeata. Müll. . . . . . . Seite $7 \mathbf{r}$ Helix lucida. Mihi.

Helix fascicularis. Geoffroy. . . . . . 74 Helix bidentata. Chemniz. •. . . . . . 77 Helix Cobresiana. Mihi. . . . . . 79 **** Rotundatae imperforatæ.

Helix limacina. Mihi. . . . . . . . $8 I$ Helix limacoides. Mihi. . . . . . . 85 Helix vivipara. Linn. . . . . . . . 86 Helix nemoralis. Linn. . . . . . . . 89 Helix hortensis. Múll. . . . . • . . 9 I ***** Ovatæ imperforatæ.

Helix ftagnalis. Linn. . . . . . . . . 93

Helix putris. Linn. . . . . . . . . 96

Helix obscura. Müll. • . . • • . . . 98

Helix buccinata. Mihi. . . . . . . . 100

Helix lubrica. Martini. . . . . . . I02

Helix tentaculata. Linn. . . . . . . . 103

Helix auricularia. Linn. . . . . . . . I05

Helix Carychium. Müll. . . . . . . . I07

Helix Corvus. Schröter. . . . . . . 109

Nerita.

Nerita valvata. Müll. . . . . . : . III

$\mathrm{P}$ atella.

*** Mucronatæ, vertice acuminato recurvo:

Patella lacustris. Linn. . . . . . . . : : II4

Patella fluviatilis. Müll. • • • • • • . I16 

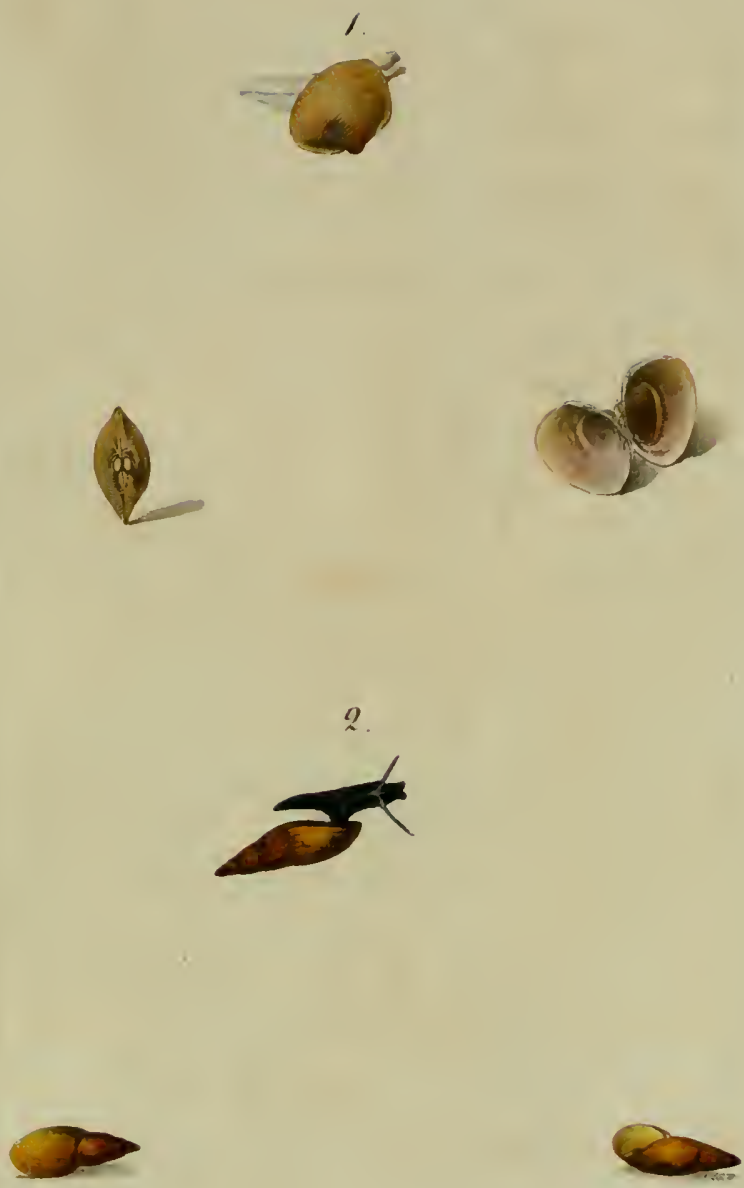

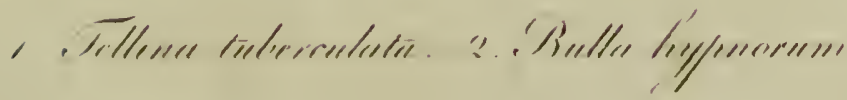



3.

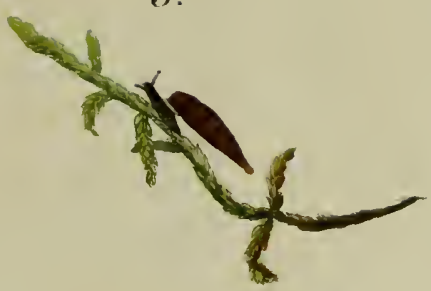

2

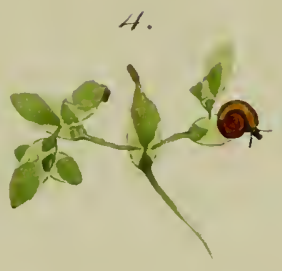

$-$

(4)

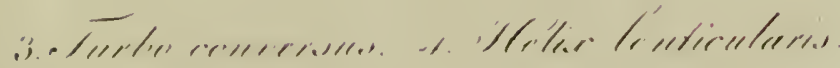





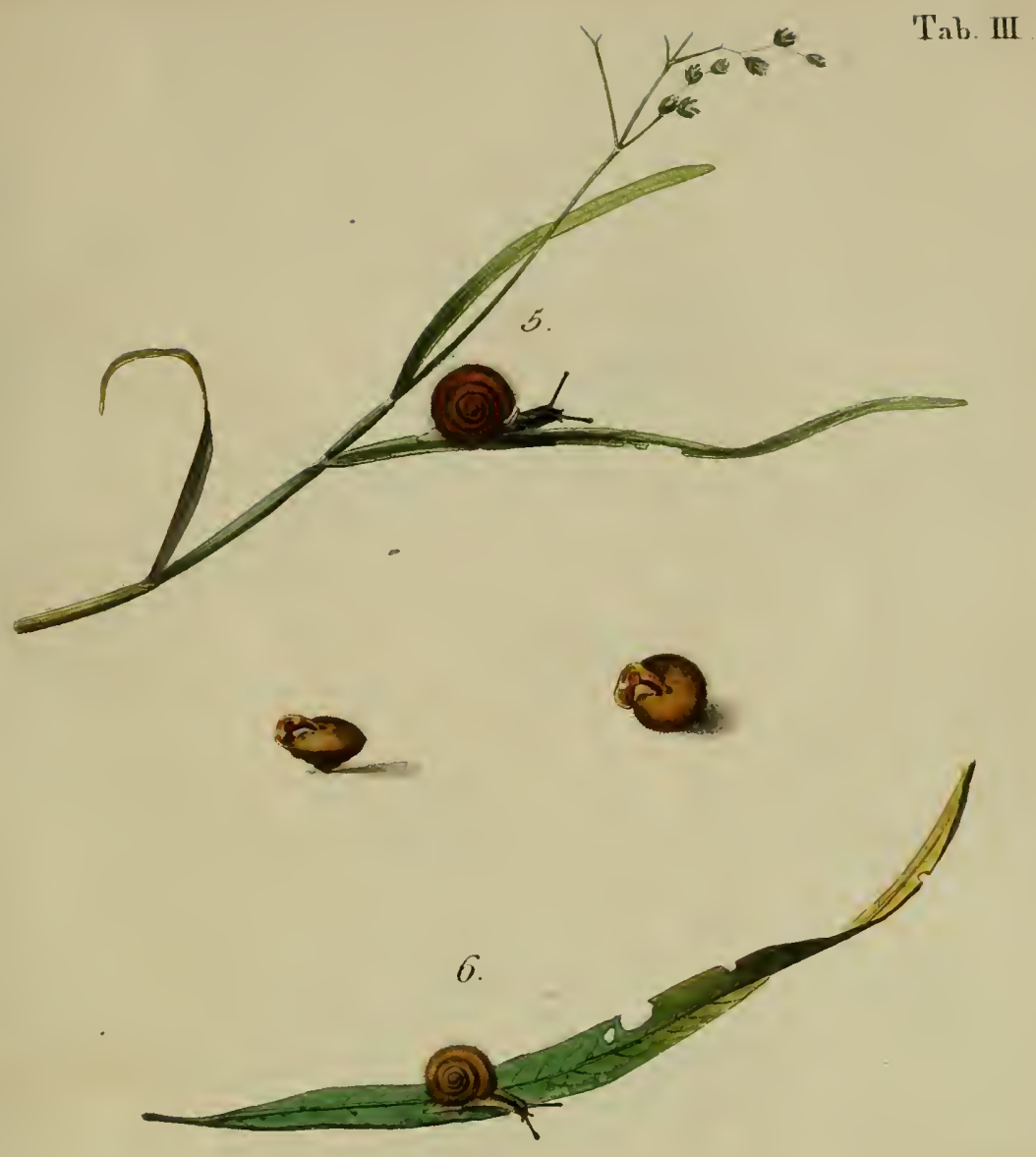

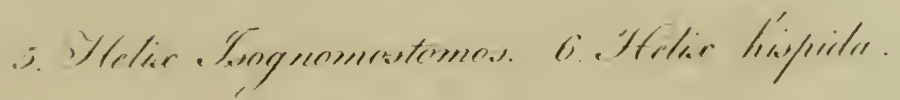



'Yals. II'.
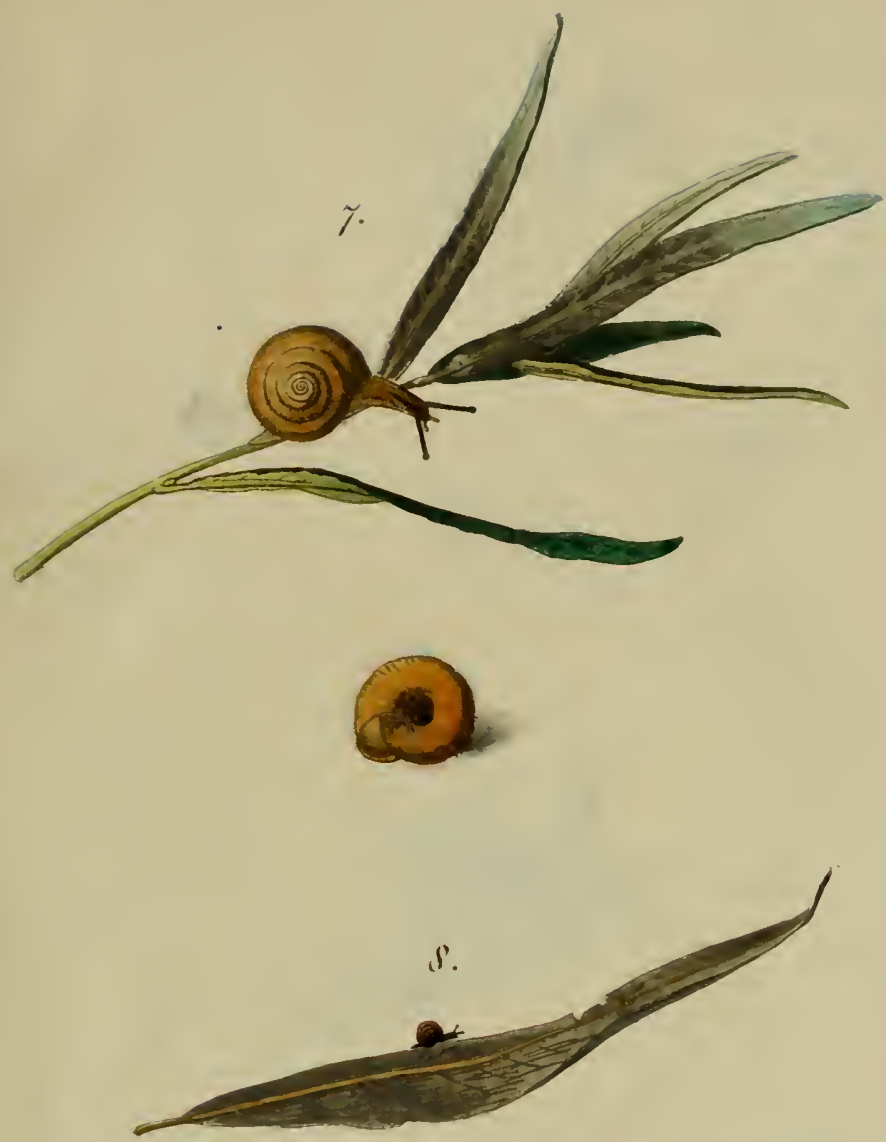

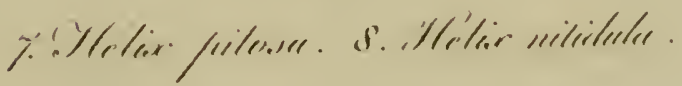




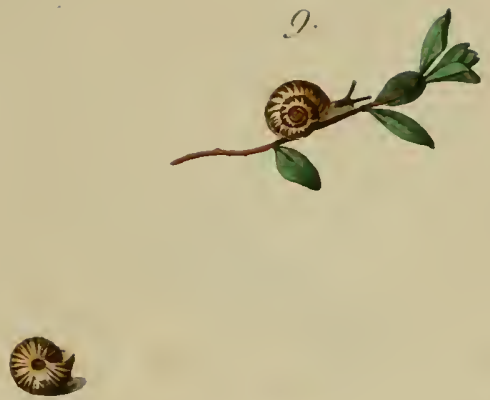

Sini
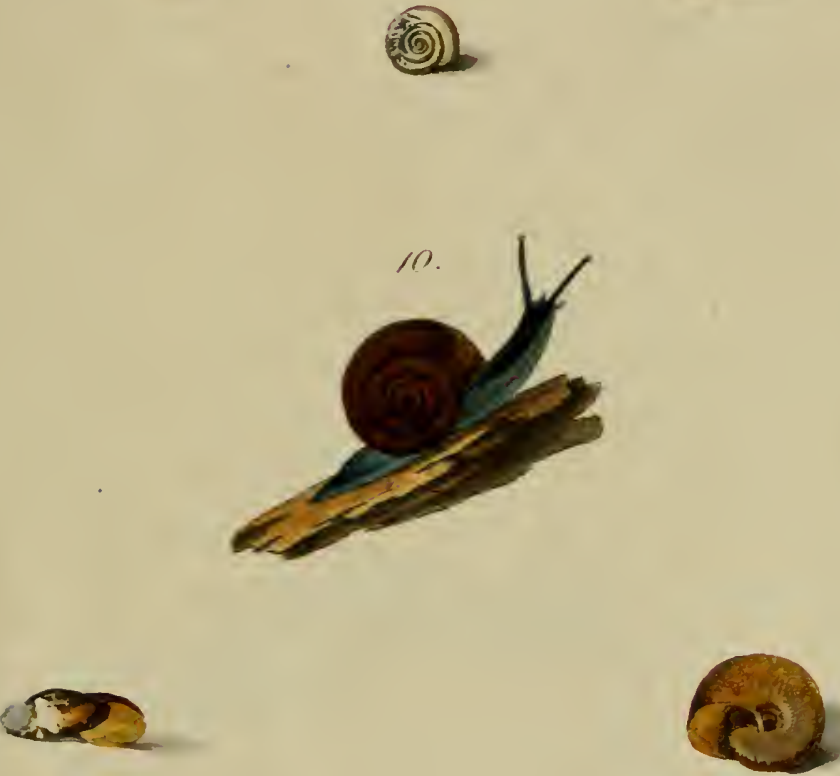

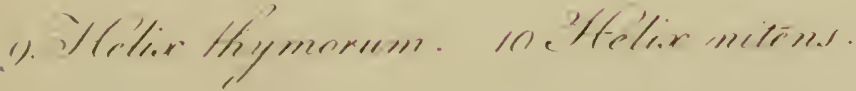



Tab. VI.
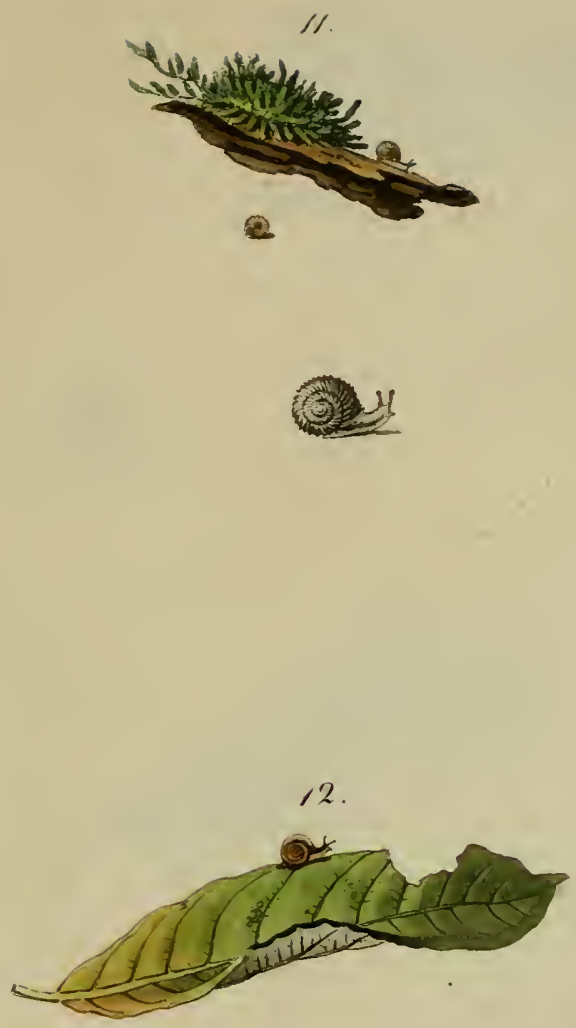

22

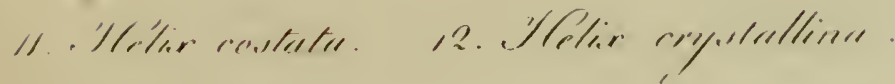




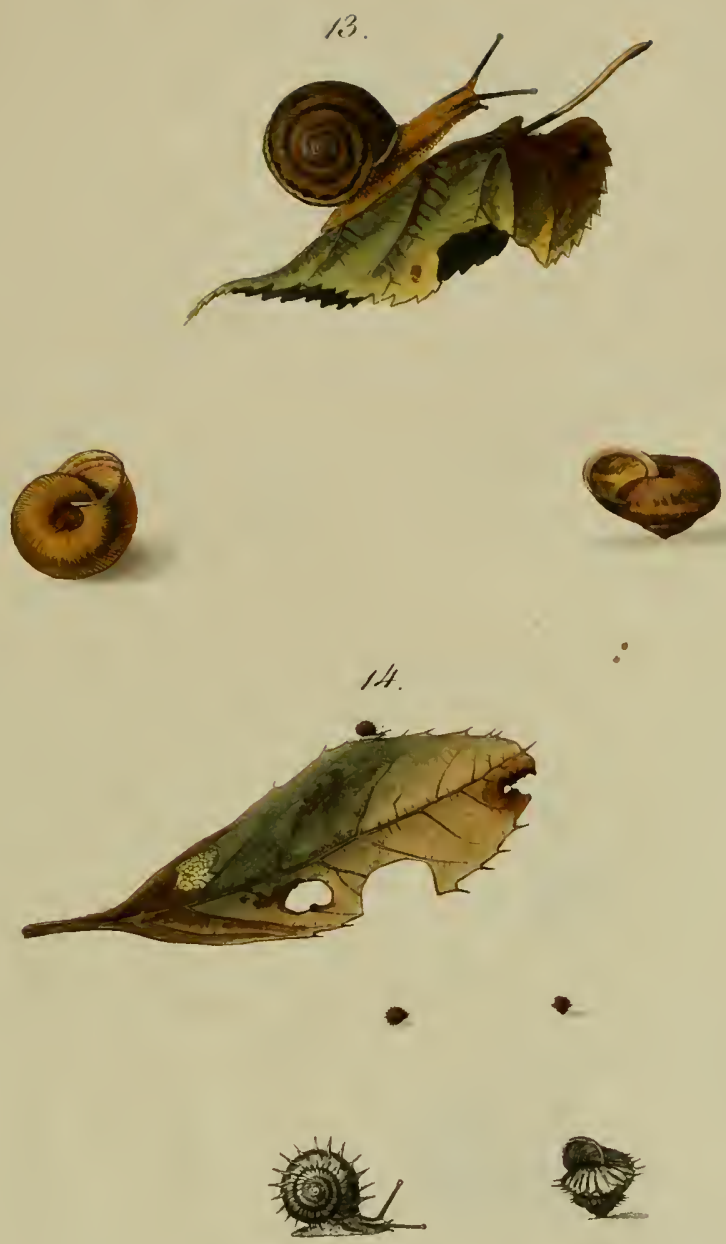

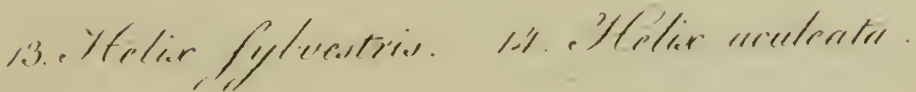



Tab.VII

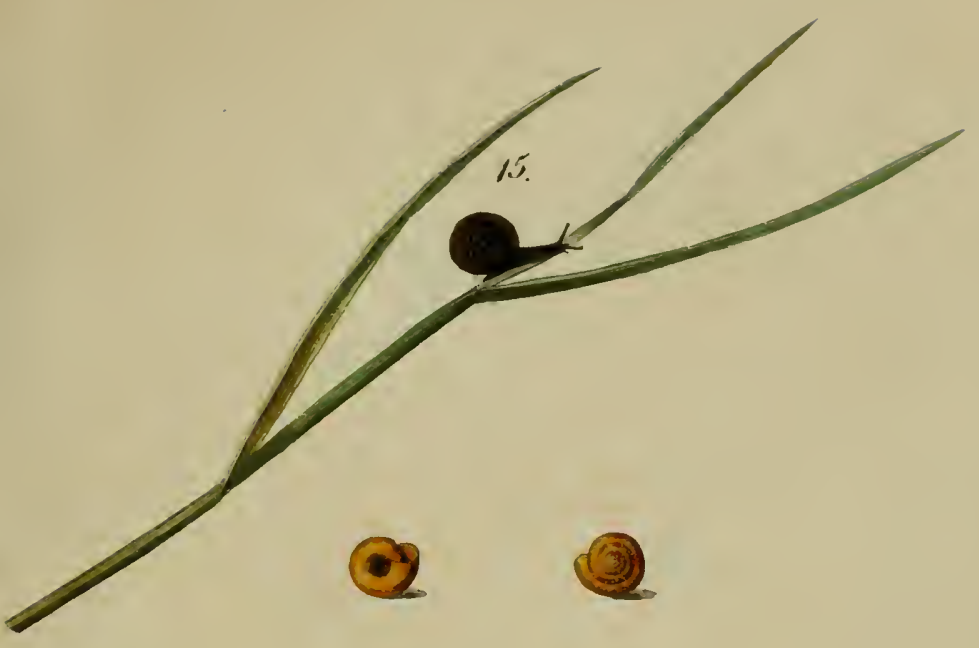

16

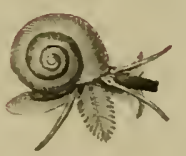

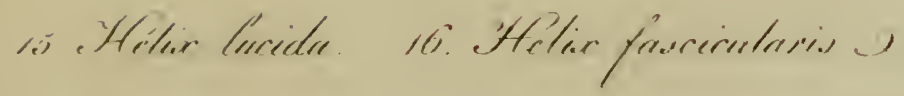



Tab IX
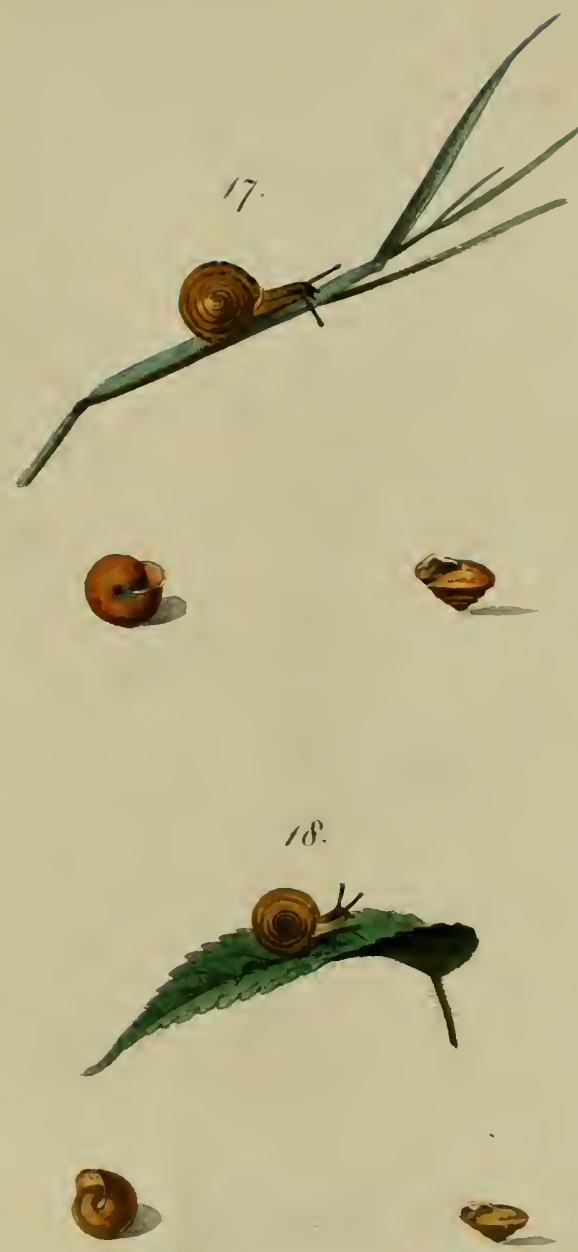

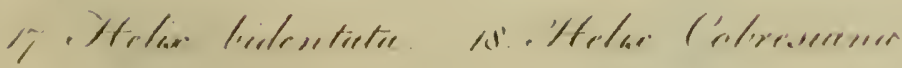





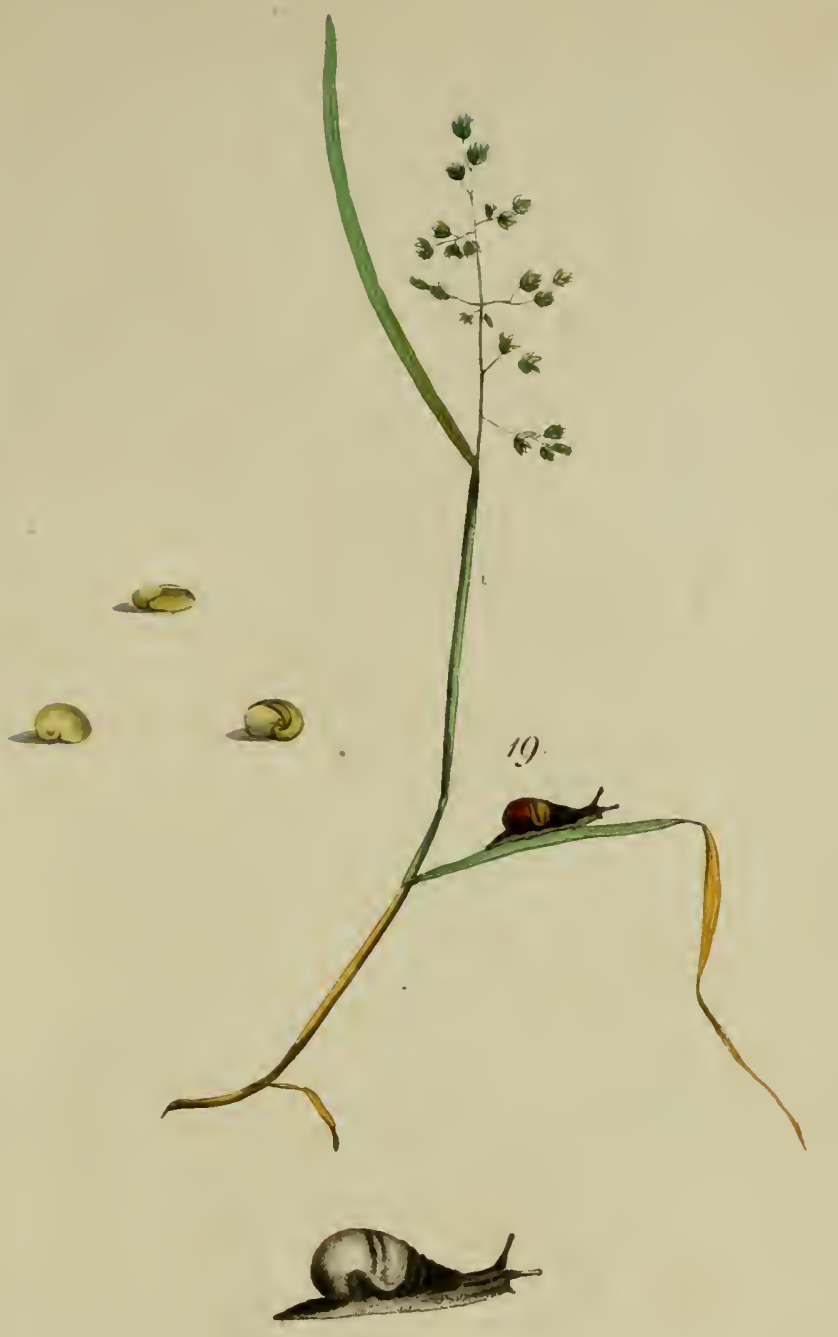

Tat, X 



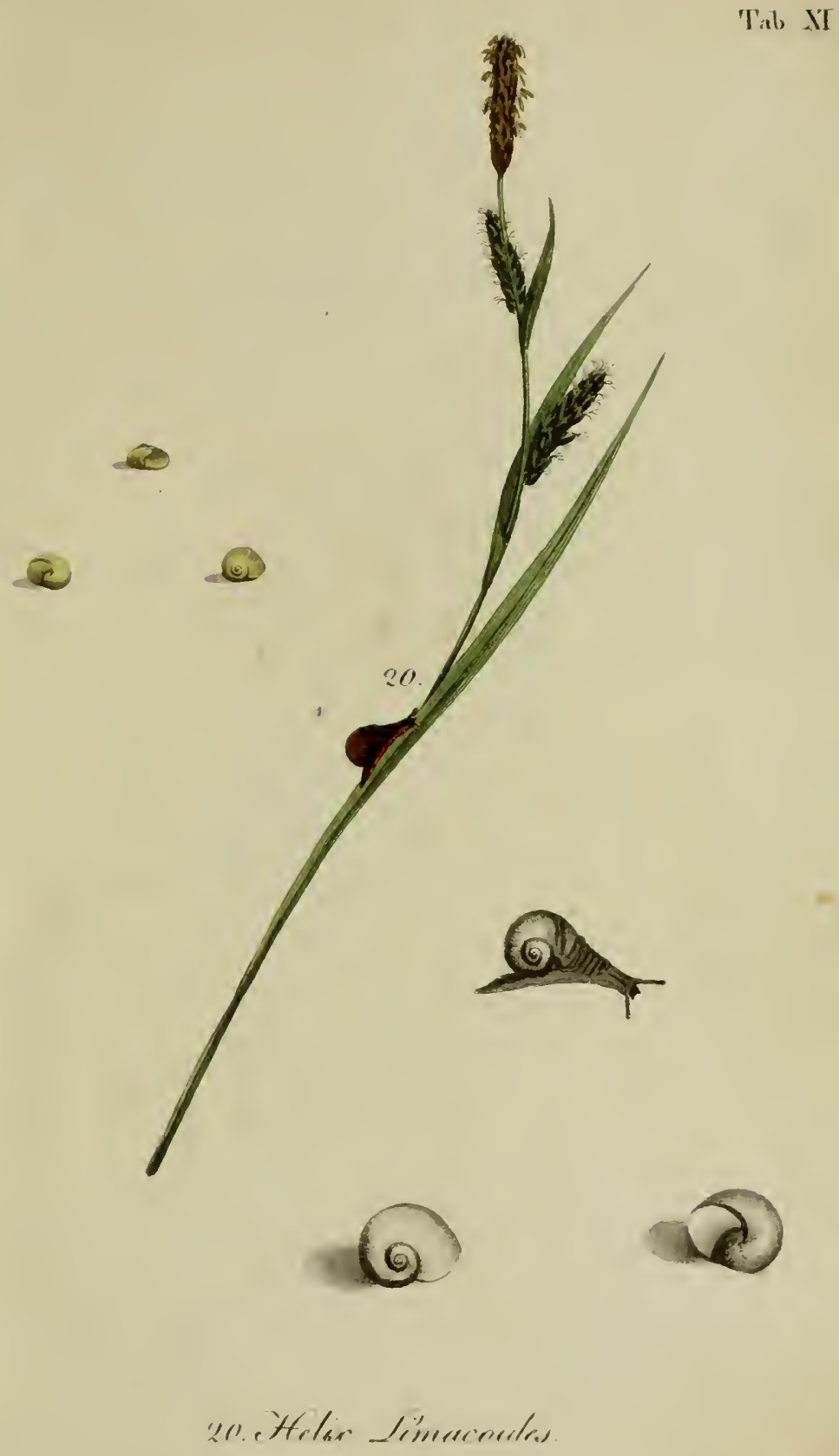



'Tab. XII.
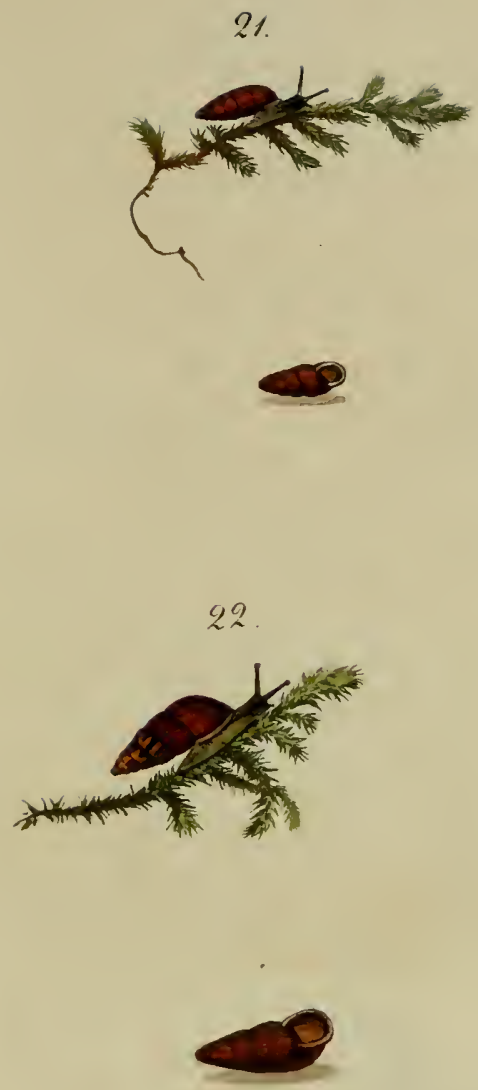

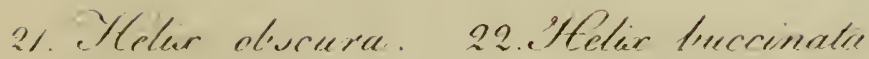




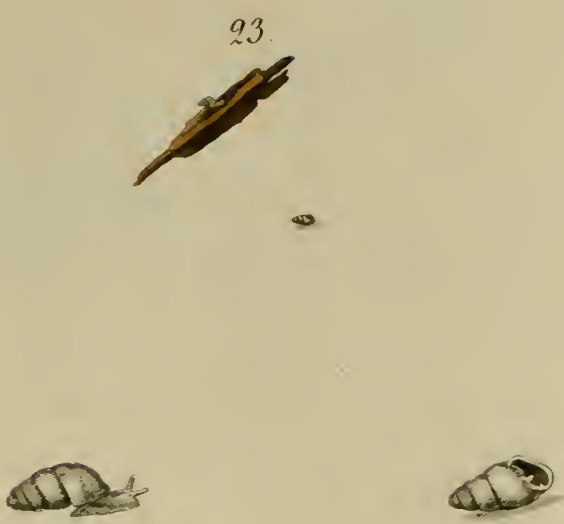

24

I

(웅

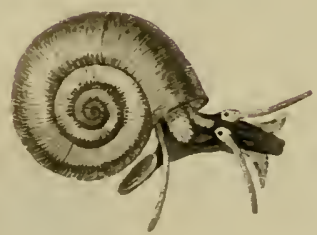

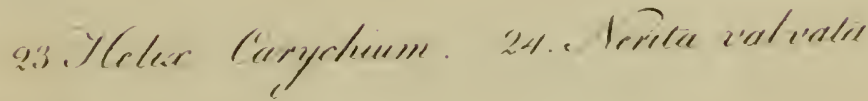



Tab ITI
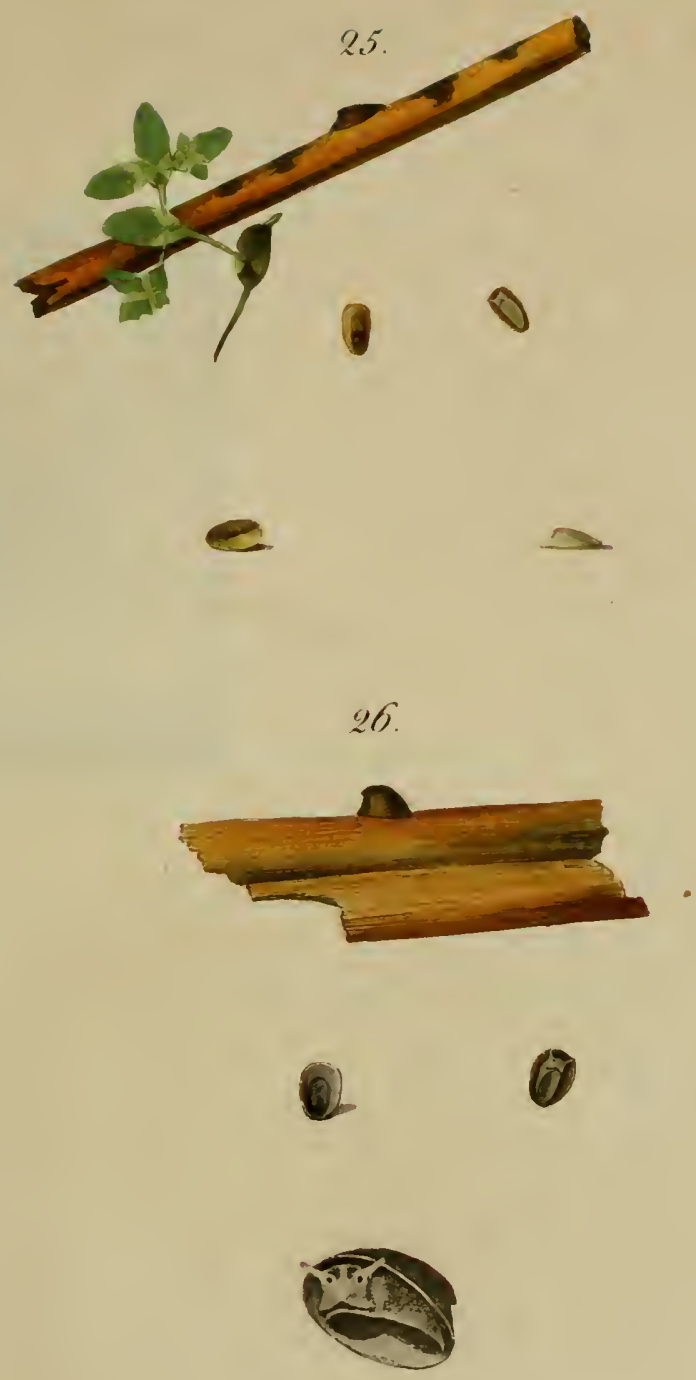

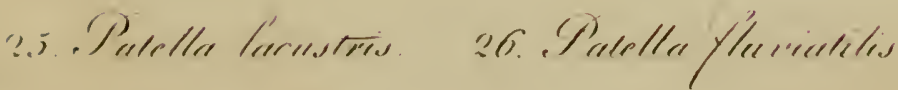





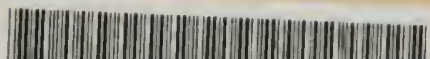 $32044072 \quad 189384$}




